\title{
Periodic motions for multi-wells potentials and layers dynamic for the vector Allen-Cahn equation
}

\author{
Giorgio Fusco*
}

\begin{abstract}
We consider a nonnegative potential $W$ that vanishes on a finite set and study the existence of periodic orbits of the equation

$$
\ddot{u}=W_{u}(u), \quad t \in \mathbb{R},
$$

that have the property of visiting neighborhoods of zeros of $W$ in a given finite sequence. We give conditions for the existence of such orbits. After introducing the new variable $x=\epsilon t, \epsilon>0$ small, these orbits correspond to stationary solutions of the parabolic equation

$$
u_{t}=u_{x x}-W_{u}(u), \quad x \in(0,1), t>0,
$$

with periodic boundary conditions.

In the second paper of the paper we study solutions of this equation that, as the stationary solutions, have a layered structure.We derive a system of ODE that describes the dynamics of the layers and show that their motion is extremely slow.
\end{abstract}

Keywords: Periodic motion, Layers dynamics, Singular perturbations.

\section{Introduction}

Let $W: \mathbb{R}^{m} \rightarrow \mathbb{R}$ be a nonnegative $C^{4}$ potential that satisfies

$$
0=W(a)<W(u), a \in A, u \in \mathbb{R}^{m} \backslash A
$$

where $A \subset \mathbb{R}^{m}$ is a finite set with at least two distinct elements. We consider the mechanical system

$$
u^{\prime \prime}=W_{u}(u), W_{u}(u)=\left(\frac{\partial W}{\partial u_{1}}(u), \ldots, \frac{\partial W}{\partial u_{m}}(u)\right)^{\top},
$$

and, given a small number $\delta>0$ and $N \geq 2$ points $a_{1}, \ldots, a_{N} \in A$ with $a_{j} \neq a_{j+1}$, $j=1, \ldots, N-1$ and $a_{N} \neq a_{1}$ we study the existence of periodic solutions $u^{T}: \mathbb{R} \rightarrow \mathbb{R}^{m}$ of (1.1) that satisfies

$$
u^{T}\left(t_{j}\right) \in B_{\delta}\left(a_{j}\right), \quad j=1, \ldots, N
$$

for some $t_{1}<\ldots<t_{N} \in[0, T), T>0$ the period of $u^{T}$.

We also require that $u^{T}$ remains away from $A \backslash\left\{a_{1}, \ldots, a_{N}\right\}$. In Fig. 1 we sketch a situation where $A$ has four elements and $N=6$.

${ }^{*}$ Dipartimento di Matematica Pura ed Applicata, Università degli Studi dell'Aquila, Via Vetoio, 67010 Coppito, L'Aquila, Italy; e-mail:fusco@univaq.it 


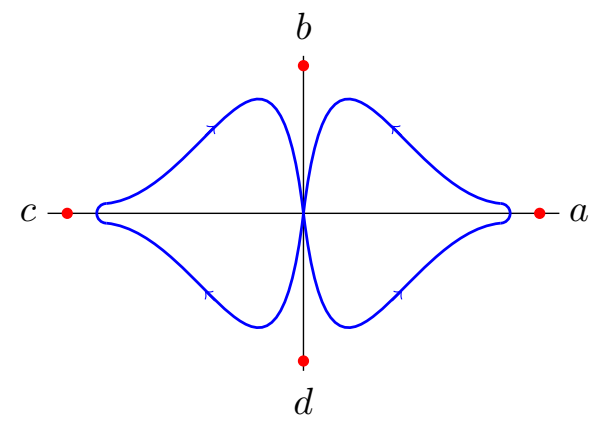

Figure 1: $A=\{a, b, c, d\}, N=6$ and $a_{1}=a, a_{2}=b, a_{3}=d, a_{4}=c, a_{5}=b, a_{6}=d$.

If $A=\left\{a_{-}, a_{+}\right\}$with $a_{-} \neq a_{+}$the problem is well understood, see for example [9], 2] and under minimal assumption on $W$, there is a $T_{0}>0$ such that, for each $T \geq T_{0}$, there exists a $T$-periodic solution $u^{T}$ that oscillates twice for period on the same trajectory with extreme at $u^{T}(0) \in B_{\delta}\left(a_{-}\right)$and $u^{T}(T / 2) \in B_{\delta}\left(a_{+}\right)$and

$$
\lim _{T \rightarrow+\infty} \delta=0 .
$$

Moreover the speed vanishes at $t=0$ and $t=T / 2$. For this reason $u^{T}$ is called a brake orbit. The existence of brake orbits that oscillate between neighborhoods of two minima of the potential has been extended also to an infinite dimensional setting where $W$ is replaced by a functional $J: \mathcal{H} \rightarrow \mathbb{R}(\mathcal{H}$ a suitable function space) with two distinct global minimizers $\bar{u}_{-} \neq \bar{u}_{+} \in \mathcal{H}$ that correspond to the minima $a_{-}, a_{+}$of $W$, see [1], [23] and [34].

The case $\sharp A \geq 3$ is open and its analysis in full generality is probably very difficult. We study the problem under generic assumptions that we now describe. We note that if a periodic solutions $u^{T}$ with the properties listed above exists for all $\delta>0$ small we can expect that there is $t_{j}^{*} \in\left(t_{j}, t_{j+1}\right)$ such that, at least along a subsequence, it results

$$
\lim _{T \rightarrow+\infty} u^{T}\left(t-t_{j}^{*}\right)=\bar{u}_{j}(t)
$$

where $\bar{u}_{j}$ is a heteroclinic solution of (1.1) that connects $a_{j}$ to $a_{j+1}$. Therefore a natural assumption in the study of the above question is

$\mathbf{H}_{1}$ For each $j=1, \ldots, N$ there exists a solution $\bar{u}_{j}$ of (1.1) which is a minimizer of the action $J(u)=\int_{\mathbb{R}}\left(\frac{1}{2}\left|u^{\prime}\right|^{2}+W(u)\right) d s$ in the class of maps that connect $a_{j}$ to $a_{j+1}$ $\left(a_{N+1}=a_{1}\right)$.

Remark. $A=\left\{a_{-}, a_{+}\right\}, a_{-} \neq a_{+}$implies the existence of a solution $\bar{u}$ of (1.1) that connects $a_{-}$to $a_{+}$[2], 30], 35], [36], 39]. If $A$ contains more than two elements, the existence of a heteroclinic solution $\bar{u}$ that connects $a_{-} \in A$ to $a_{+} \in A, a_{-} \neq a_{+}$is not guaranteed. A sufficient condition for $\bar{u}$ to exist it that

$$
\sigma_{a_{-}, a_{+}}<\sigma_{a_{-}, a}+\sigma_{a, a_{+}}, \quad a \in A \backslash\left\{a_{-}, a_{+}\right\},
$$

where, for $a \neq b \in A$,

$$
\sigma_{a, b}=\inf _{u \in \mathscr{A}_{a, b}} J(u), \quad \mathscr{A}_{a, b}=\left\{u \in W_{\text {loc }}^{1,2}: u \text { connects } a \text { to } b\right\} .
$$


We also assume the following nondegeneracy conditions:

$\mathbf{H}_{2}$ The hessian matrix $W_{u u}\left(a_{j}\right), j=1, \ldots, N$ has distinct positive eigenvalues.

$\mathbf{H}_{3}$ The zero eigenvalue of the operator $L_{j}: W^{2,2}\left(\mathbb{R} ; \mathbb{R}^{m}\right) \rightarrow L^{2}\left(\mathbb{R} ; \mathbb{R}^{m}\right)$ defined by

$$
L_{j} v=-v^{\prime \prime}+W_{u u}\left(\bar{u}_{j}\right) v,
$$

is simple.

Remark. For each $s_{0} \in \mathbb{R}, \bar{u}_{j}\left(\cdot-s_{0}\right)$ is a solution of (1.1). Therefore by differentiating with respect to $s_{0}$ we get $\bar{u}_{j}^{\prime \prime \prime}=W_{u u}\left(\bar{u}_{j}\right) \bar{u}_{j}^{\prime}$. That is 0 is an eigenvalue of $L_{j}$ and $\bar{u}_{j}^{\prime}$ a corresponding eigenvector.

The smoothness of $W$ and $\mathbf{H}_{2}$ imply, see Lemma 4.1, the existence of the limits

$$
\lim _{s \rightarrow-\infty} \frac{\bar{u}_{j}(s)-a_{j}}{\left|\bar{u}_{j}(s)-a_{j}\right|}=z_{j}^{-}, \quad \lim _{s \rightarrow \infty} \frac{\bar{u}_{j-1}(s)-a_{j}}{\left|\bar{u}_{j-1}(s)-a_{j}\right|}=z_{j}^{+},
$$

where $z_{j}^{ \pm}$are eigenvectors of the matrix $W_{u u}\left(a_{j}\right)$. We define $\bar{u}_{j}$ also for $j=N+1$ and $s<0$ and for $j=0$ and $s>0$ by setting

$$
\begin{aligned}
& \bar{u}_{N+1}(s)-a_{N+1}=\bar{u}_{1}(s)-a_{1}, \quad s<0, \\
& \bar{u}_{0}(s)-a_{1}=\bar{u}_{N}(s)-a_{N+1}, \quad s>0,
\end{aligned}
$$

and observe that these definitions imply

$$
z_{1}^{+}=z_{N+1}^{+}, \quad z_{N+1}^{-}=z_{1}^{-} .
$$

Note that from $\mathbf{H}_{2}$ the eigenvectors $z_{j}^{-}$and $z_{j}^{+}$are either parallel or orthogonal.

We prove the following

Theorem 1.1. Assume $\mathbf{H}_{1}-\mathbf{H}_{3}$ and

$\mathbf{H}_{4} z_{j}^{-}= \pm z_{j}^{+}, \quad j=1, \ldots, N$,

Then, the condition

$$
z_{j}^{-} \cdot z_{j}^{+}=z_{j+1}^{-} \cdot z_{j+1}^{+}, \quad j=1, \ldots, N,
$$

is necessary and sufficient for the existence of a family $u^{T}: \mathbb{R} \rightarrow \mathbb{R}^{m}, T \geq T_{0}$, for some $T_{0}>0$ of T-periodic solutions of (1.1) that satisfy:

(i) There exist numbers $0 \leq \xi_{1}<\xi_{2}<\ldots<\xi_{N}<1$ such that

$$
\begin{aligned}
& \lim _{T \rightarrow+\infty} \xi_{j}-\xi_{j-1}=\frac{1 / \mu_{j}}{\sum_{h} 1 / \mu_{h}}, \quad\left(\xi_{0}=\xi_{N}-1\right) \\
& \quad \text { and } \\
& \lim _{T \rightarrow+\infty} u^{T}\left(t+\xi_{j} T\right)=\bar{u}_{j}(t),
\end{aligned}
$$

uniformly in compacts.

(ii) Given $\delta>0$ there are $t_{\delta}>0$ and $T_{\delta}>0, t_{\delta}$ independent of $T$, such that

$$
\begin{aligned}
& u^{T}(t) \in B_{\delta}\left(a_{j}\right), \text { for } t \in\left(\xi_{j-1} T+t_{\delta}, \xi_{j} T-t_{\delta}\right), \\
& j=1, \ldots, N, \quad T \geq T_{\delta} .
\end{aligned}
$$


(iii) There exist $k_{0}, K_{0}$ independent of $T$ such that

$$
\begin{aligned}
& \left\|u^{T}-u^{T, \xi}\right\|_{W^{1,2}\left((0, T) ; \mathbb{R}^{m}\right)} \leq K_{0} e^{-k_{0} T} e^{-\frac{T}{2 \sum_{h} \frac{1}{\mu_{h}}}}, \\
& \left|J_{(0, T)}\left(u^{T}\right)-J_{(0, T)}\left(u^{T, \xi}\right)\right| \leq K_{0} e^{-k_{0} T} e^{-\frac{T}{2 \sum_{h} \frac{1}{\mu_{h}}}} .
\end{aligned}
$$

where $u^{T, \xi}$ is defined in (1.13) and $J_{(0, T)}(v)=\int_{0}^{T}\left(\frac{\left|v^{\prime}\right|^{2}}{2}+W(v)\right) d t$.

In the scalar case $m=1$ the condition $z_{j}^{-}= \pm z_{j}^{+}$is automatically satisfied. In the vector case $m>1, \mathbf{H}_{4}$ should be regarded as a generic assumption. Indeed it can be shown that if $\bar{u}$ is a solution of (1.1) that converges to $a \in A$, for $s \rightarrow-\infty$ or for $s \rightarrow \infty$ then, generically, the ratio $\frac{\bar{u}(s)-a}{|\bar{u}(s)-a|}$ converges to an eigenvector of $W_{u u}(a)$ that corresponds to the smallest eigenvalue of $W_{u u}(a)$. A situation where the condition $z_{j}^{-}= \pm z_{j}^{+}$may automatically hold in the vector case is when $W$ is invariant under a group of symmetries. For instance this is the case when $W: \mathbb{R}^{m} \rightarrow \mathbb{R}$ is invariant under the Dihedral group $D_{K}$ of the symmetries of a regular polygon with $K$ sides and $A=\{W=0\}$ has exactly $K$ distinct elements. The situations corresponding to $z_{j}^{-} \cdot z_{j}^{+}= \pm 1$ are illustrated in Fig, 2 ,

In the scalar case $m=1$, in agreement with phase plane analysis, periodic solutions whose existence can the established via Theorem 1.1 are necessarily of brake type oscillating between neighborhoods of consecutive elements of $A$. This follows from the fact that, since $A \subset \mathbb{R}$ is ordered, the necessary condition (1.5) can not be satisfied if the sequence $a_{1}, \ldots, a_{N}$ includes three or more distinct elements of $A$. Assume for example that $W(u)=\frac{1}{4} u^{2}\left(1-u^{2}\right)^{2}$. Then $A=\{-1,0,1\}$ and $W^{\prime \prime}( \pm 1)=2, W^{\prime \prime}(0)=\frac{1}{2}$. Phase plane analysis shows that there exist heteroclinic connections $\bar{u}$ and $\bar{v}$ connecting -1 to 0 and 0 to 1 respectively. For $N=4$ consider the sequence $a_{1}=-1, a_{2}=0, a_{3}=1$ and $a_{4}=0$ with the associated sequence of connections $\bar{u}_{1}=\bar{u}, \bar{u}_{2}=\bar{v}, \bar{u}_{3}=\bar{v}(-\cdot)$ and $\bar{u}_{4}=\bar{u}(-\cdot)$. In this case we have in particular

$$
\begin{aligned}
& z_{2}^{-}=1, \quad z_{2}^{+}=-1, \\
& z_{3}^{-}=z_{3}^{+}=-1
\end{aligned}
$$

It follows

$$
z_{2}^{-} \cdot z_{2}^{+} \neq z_{3}^{-} \cdot z_{3}^{+}
$$

This shows that the necessary and sufficient condition formulated in Theorem 1.1 is not satisfied and we conclude that the periodic orbit $u^{T}$ does not exist and indeed inspection of the phase plane confirms that there is no periodic orbit of (1.1) that visit in sequence $\delta$-neighborhoods of $a_{1}, a_{2}, a_{3}, a_{4}$. Note also that this example shows that the product $\varsigma_{j}:=z_{j}^{-} \cdot z_{j}^{+}$can assume both values 1 and -1 .

Assume now $m \geq 2$ and that $W: \mathbb{R}^{m} \rightarrow \mathbb{R}$ satisfies $0=W(a)<W(u)$ for $a \in\left\{a_{1}, a_{2}\right\}$, $u \in \mathbb{R}^{m} \backslash\left\{a_{1}, a_{2}\right\}$ with $a_{1} \neq a_{2}$ nondegenerate zeros of $W$ and assume that there is a nondegenerate connection $\bar{u}$ connecting $a_{1}$ to $a_{2}$. Consider the sequence $a_{1}, a_{2}(N=2)$ and the associate sequence of connections $\bar{u}_{1}=\bar{u}, \bar{u}_{2}=\bar{u}(-\cdot)$. In this case it results

$$
z_{1}^{-}=z_{1}^{+}, \quad z_{2}^{-}=z_{2}^{+},
$$

and Theorem 1.1 implies the existence of a periodic orbit $u^{T}$ that, for $T$ sufficiently large alternates between $\delta$-neighborhoods of $a_{1}$ and $a_{2}$. Beside this simple situation that extends to the case $m \geq 2$ well known results [19], 24] valid for the scalar case, in the vector case 


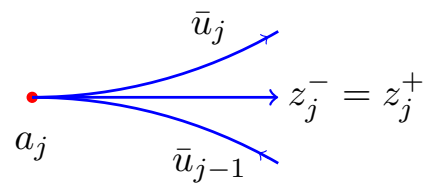

a)

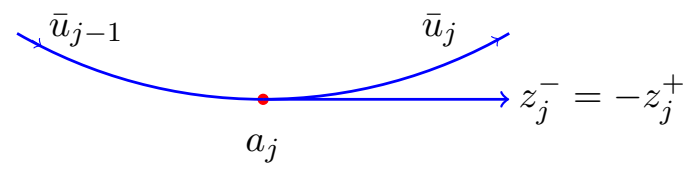

b)

Figure 2 : a) $z_{j}^{-} \cdot z_{j}^{+}=1$; b) $z_{j}^{-} \cdot z_{j}^{+}=-1$.

$m \geq 2$, depending on the sign of the products in (1.5) and on the structure of the set of connections between the elements of $A$, various type of periodic solutions of (1.1) can be deduced from Theorem 1.1. Even in the case where $A=\left\{a_{-}, a_{+}\right\}$has only two elements, if it happens [4] that there are two distinct orbits connecting $a_{-}$to $a_{+}$, say $\bar{u} \neq \bar{v}$ that satisfy the condition

$$
\lim _{s \rightarrow \pm \infty} \frac{\bar{u}(s)-a_{ \pm}}{\left|\bar{u}(s)-a_{ \pm}\right|}=\lim _{s \rightarrow \pm \infty} \frac{\bar{v}(s)-a_{ \pm}}{\left|\bar{v}(s)-a_{ \pm}\right|}=z^{ \pm}
$$

Theorem 1.1 implies the existence of infinitely many periodic solutions of (1.1) with different structure. Indeed (1.6) implies that, independently of the path chosen to travel from $a_{-}$to $a_{+}$and viceversa, the value of the products in (1.5) is always 1 . Therefore if we represent the connections $\bar{u}, \bar{u}(-\cdot), \bar{v}, \bar{v}(-\cdot)$ with the four symbols $+p,-p,+q,-q$, then Theorem 1.1 implies the existence of a periodic solution for each sequence $\left\{\omega_{h}\right\}_{h=1}^{N}$ which has $N$ even and satisfies

$$
\omega_{h} \in\{+p,-p,+q,-q\}, \quad \operatorname{sign}\left(\omega_{h}\right) \operatorname{sign}\left(\omega_{h+1}\right)=-1, \quad h=1, \ldots
$$

In this situation we can even conjecture that, as limits of sequences of periodic solutions corresponding to sequences $\left\{\omega_{h}\right\}_{h=1}^{N}$ with $N \rightarrow \infty$, there exist chaotic aperiodic solutions of (1.1) associated to infinite sequences $\left\{\omega_{h}\right\}_{h=1}^{\infty}$ that satisfy (1.7). Similar considerations apply when the graph with vertices the elements of $A$ and edges the connections among them has a connected component that contains three or more elements of $A$. This happens, for instance, if $\sharp A$ is odd.

We denote $\mu_{j} \pm^{2}$ the eigenvalue of $W_{u u}\left(a_{j}\right)$ associated to eigenvectors $z_{j}^{ \pm}$in (1.4). From $\mathbf{H}_{2}$ and (1.4) it follows, see Lemma 4.1, that there exist maps $\bar{w}_{j}^{ \pm}$and constants $\bar{K}_{j}^{ \pm}>0$, $K_{j}^{ \pm}>0$ and $\hat{\mu}_{j}^{-}>\mu_{j}^{-}, \hat{\mu}_{j}^{+}>\mu_{j+1}^{+}$such that

$$
\begin{aligned}
& \bar{u}_{j}(s)-a_{j}=\bar{K}_{j}^{-} z_{j}^{-} e^{\mu_{j}^{-} s}+\bar{w}_{j}^{-}(s), \quad s \leq 0, \\
& \bar{u}_{j}(s)-a_{j+1}=\bar{K}_{j}^{+} z_{j+1}^{+} e^{-\mu_{j+1}^{+} s}+\bar{w}_{j}^{+}(s), \quad s \geq 0, \\
& \left|\bar{w}_{j}^{-}(s)\right|,\left|D^{k} \bar{w}_{j}^{-}(s)\right| \leq K_{j}^{-} e^{\hat{\mu}_{j}^{-} s}, \quad s \leq 0, k=1,2,3, \\
& \left|\bar{w}_{j}^{+}(s)\right|,\left|D^{k} \bar{w}_{j}^{+}(s)\right| \leq K_{j}^{+} e^{-\hat{\mu}_{j}^{+} s}, \quad s \geq 0, k=1,2,3 .
\end{aligned}
$$


If also $\mathbf{H}_{4}$ is satisfied (1.8) $)_{1}$ becomes

$$
\begin{aligned}
& \bar{u}_{j}(s)-a_{j}=\bar{K}_{j}^{-} z_{j}^{-} e^{\mu_{j} s}+\bar{w}_{j}^{-}(s), \quad s \leq 0, \\
& \bar{u}_{j}(s)-a_{j+1}=\bar{K}_{j}^{+} z_{j+1}^{+} e^{-\mu_{j+1} s}+\bar{w}_{j}^{+}(s), \quad s \geq 0,
\end{aligned}
$$

where $\mu_{j}^{2}$ is the eigenvalue of $W_{u u}\left(a_{j}\right)$ associated to the eigenvectors $z_{j}^{-}$and $z_{j}^{+}$.

The exponential decay of $\bar{u}_{j}$ in (1.8) suggests that by patching together translates of the maps $\bar{u}_{j}-a_{j}$ and $\bar{u}_{j}-a_{j+1}, j=1, \ldots, N$ we can construct an $N$-dimensional manifold of periodic maps that are approximate solutions of (1.1) in the sense that satisfy (1.1) up to an exponentially small error that depends on the size of the translations. The idea is then to construct exact periodic solutions of (1.1) by small perturbations of maps on the approximate manifold. Since the period of the sought periodic solutions is unknown we prefer to rescale equation (1.1) by the change of variable $t=\frac{x}{\epsilon}, \epsilon>0$ small and look for periodic solutions of period 1 for the rescaled equation

$$
\epsilon^{2} u_{x x}=W_{u}(u) .
$$

Set $\mu_{j}=\frac{1}{2}\left(\mu_{j}^{-}+\mu_{j}^{+}\right)$. For $\rho>0$ small, we define

$$
\Xi_{\rho}:=\left\{\xi \in \mathbb{R}^{N}: 0 \leq \xi_{1}, \xi_{j}-\xi_{j-1}>\frac{\rho}{\mu_{j}}, j=1, \ldots, N, \xi_{0}=\xi_{N}-1<\xi_{1}\right\}
$$

and focus on the manifold $\mathcal{M}=\left\{u^{\xi}: \xi \in \Xi_{\rho}\right\}$ where $u^{\xi}$ is defined by

$$
u^{\xi}(x)=\sum_{n<0} \sum_{h=1}^{N}\left(\bar{u}_{h}\left(\frac{x-n-\xi_{h}}{\epsilon}\right)-a_{h+1}\right)+a_{1}+\sum_{n \geq 0} \sum_{h=1}^{N}\left(\bar{u}_{h}\left(\frac{x-n-\xi_{h}}{\epsilon}\right)-a_{h}\right) .
$$

$\mathcal{M}$ is a manifold of 1-periodic maps which, in each interval $\left(\hat{\xi}_{j-1}+n, \hat{\xi}_{j}+n\right), n \in \mathbb{Z}$ $\left(\hat{\xi}_{j}=\frac{\xi_{j+1}+\xi_{j}}{2}, j=1, \ldots, N ; \xi_{N+1}=\xi_{1}+1\right)$, aside from an error of $\mathrm{O}\left(e^{-\frac{\alpha \rho}{\epsilon}}\right), \alpha>0$ a constant independent of $\epsilon$, coincide with $\bar{u}_{j}\left(\frac{x-n-\xi_{j}}{\epsilon}\right)$. Since $\bar{u}_{j}\left(\frac{x-n-\xi_{j}}{\epsilon}\right)$ is a solution of (1.9) it follows, see also Lemma 2.7

$$
\mathscr{F}\left(u^{\xi}\right):=\epsilon^{2} u_{x x}^{\xi}-W_{u}\left(u^{\xi}\right)=\mathrm{O}\left(e^{-\frac{\alpha \rho}{\epsilon}}\right) .
$$

In the following $\alpha>0$ and $C>0$ denote generic constants independent of $\epsilon>0$ and $\xi \in \Xi_{\rho}$.

Remark. If we set back $x=\frac{t}{T}$ with $T=\frac{1}{\epsilon}$ in the definition (1.11) of $u^{\xi}$, the rescaled version of $u^{\xi}$

$$
u^{T, \xi}(t)=\sum_{n<0} \sum_{h=1}^{N}\left(\bar{u}_{h}\left(t-n T-\xi_{h} T\right)-a_{h+1}\right)+a_{1}+\sum_{n \geq 0} \sum_{h=1}^{N}\left(\bar{u}_{h}\left(t-n T-\xi_{h} T\right)-a_{h}\right),
$$

is a $T$-periodic map and $\left\{u^{T, \xi}: \xi \in \Xi_{\rho}\right\}$ is a manifold of $T$-periodic maps.

If we set $u=u^{\xi}+v$ in (1.9) the problem of the existence of periodic solutions with the sought properties becomes the problem of finding $\xi \in \Xi_{\rho}$ and a 1-periodic map $v: \mathbb{R} \rightarrow \mathbb{R}^{m}$ that solve the equation $\epsilon^{2}\left(u_{x x}^{\xi}+v_{x x}\right)=W_{u}\left(u^{\xi}+v\right)$. The estimate (1.12) suggests to rewrite this equation as a weakly nonlinear problem

$$
\epsilon^{2} v_{x x}-W_{u u}\left(u^{\xi}\right) v=W_{u}\left(u^{\xi}\right)-\epsilon^{2} u_{x x}^{\xi}+N^{\xi}(v)=\mathrm{O}\left(e^{-\frac{\alpha \rho}{\epsilon}}\right)+N^{\xi}(v),
$$


where $N^{\xi}(v)=W_{u}\left(u^{\xi}+v\right)-W_{u}\left(u^{\xi}\right)-W_{u u}\left(u^{\xi}\right) v$ is quadratic in $v$. The difficulty with this equation is that the linear operator $L^{\xi} v=-\epsilon^{2} v_{x x}+W_{u u}\left(u^{\xi}\right) v$ is almost singular. Indeed the 0 eigenvalue of $L_{j}, j=1, \ldots, N$ manifests itself in the existence of $N$ exponentially small eigenvalues of $L^{\xi}$ with corresponding eigenspace $X^{\xi}$ approximately spanned by $u_{\xi_{1}}^{\xi}, \ldots, u_{\xi_{N}}^{\xi}$. More precisely we have $X^{\xi}=\operatorname{Span}\left(\varphi_{1}, \ldots, \varphi_{N}\right)$ where $\varphi_{j}=u_{\xi_{j}}^{\xi} /\left\|u_{\xi_{j}}^{\xi}\right\|+\mathrm{O}\left(e^{-\frac{\alpha \rho}{\epsilon}}\right), j=1, \ldots, N$ is an orthonormal basis for $X^{\xi}$ and $\|\cdot\|$ is the norm in $L^{2}\left((0,1) ; \mathbb{R}^{m}\right)$. To overcome the problem of the singularity of $L^{\xi}$ on $X^{\xi}$, a natural approach is, in the spirit of the classical method of Lapunov-Smidth and bifurcation theory, to solve (1.14) in two steps. In the first step we solve the projection of (1.14) on the orthogonal complement $X^{\xi, \perp}$ of $X^{\xi}$ :

$$
-L^{\xi} v=\sum_{j} \varphi_{j}^{\xi} c_{j}(\xi, v)+N^{\xi}(v)+W_{u}\left(u^{\xi}\right)-\epsilon^{2} u_{x x}^{\xi}
$$

where $v \in X^{\xi, \perp}$ and $c(\xi, v) \in \mathbb{R}^{N}$ is a Lagrange multiplier determined by the condition that the right hand side of (1.15) be in $X^{\xi, \perp}$. We find (Proposition 2.2) that, for each $\xi \in \Xi_{\rho}$ there are unique $v^{\xi} \in X^{\xi, \perp}, v^{\xi}=\mathrm{O}\left(e^{-\frac{\alpha \rho}{\epsilon}}\right)$ and $c(\xi)=c\left(\xi, v^{\xi}\right) \in \mathbb{R}^{N}$ that solve (1.14). The second step in the proof of Theorem 1.1 is the analysis of the bifurcation equation

$$
c(\xi)=0 .
$$

Under the assumptions of Theorem 1.1 and in particular if $\mathbf{H}_{4}$ holds, we prove that (see Theorem 2.5 and (2.28) )

$$
\begin{aligned}
& c_{j}(\xi)=\frac{2 \epsilon^{\frac{1}{2}}}{\bar{q}_{j}}\left(\varsigma_{j+1} k_{j+1} \mathscr{E}_{j+1}-\varsigma_{j} k_{j} \mathscr{E}_{j}+\mathrm{O}\left(e^{-\frac{\alpha \rho}{\epsilon}} \max _{h} \mathscr{E}_{h}\right), j=1, \ldots, N,\right. \\
& \mathscr{E}_{j}=e^{-\frac{\mu_{j}}{\epsilon}\left(\xi_{j}-\xi_{j-1}\right)},
\end{aligned}
$$

where $k_{j}=\mu_{j}^{2} \bar{K}_{j}^{-} \bar{K}_{j-1}^{+}, \varsigma_{j}=z_{j}^{-} \cdot z_{j}^{+}$and $\bar{q}_{j}^{2}=J\left(\bar{u}_{j}\right)=\int_{\mathbb{R}}\left|\bar{u}_{j}^{\prime}\right|^{2} d s$ is the energy of the connection $\bar{u}_{j}$.

On the basis of this estimate the necessity of (1.5) is quite obvious. Indeed from (1.16), if $\mathscr{E}_{j}=\max _{h} \mathscr{E}_{h}, c_{j}(\xi)=0$ is possible only if

$$
\varsigma_{j}=\varsigma_{j+1}, \text { and } \mathscr{E}_{j+1} \approx \frac{k_{j}}{k_{j+1}} \mathscr{E}_{j} .
$$

On the other hand if $\bar{\xi}$ is such that $\mathscr{E}_{j+1}=\frac{k_{j}}{k_{j+1}} \mathscr{E}_{j}$, the exponential structure of $\mathscr{E}_{h}$, $h=1, \ldots, N$, and an application of Brouwer fixed point theorem show, see Theorem 2.10, that there is a small perturbation $\xi$ of $\bar{\xi}$ that solves the bifurcation equation completing the proof of Theorem 1.1 .

Next we focus on the dynamics of the parabolic equations

$$
\begin{aligned}
& u_{t}=\mathscr{F}(u), \quad x \in(0,1), u \in W^{1,2}, \\
& \mathscr{F}(u)=\epsilon^{2} u_{x x}-W_{u}(u),
\end{aligned}
$$

(with periodic boundary conditions) in a neighborhood of the manifold $\mathcal{M}=\left\{u^{\xi}: \xi \in \Xi_{\rho}\right\}$. We observe that with $v=v^{\xi}$, (1.15) is actually equivalent to

$$
\mathscr{F}\left(\hat{u}^{\xi}\right)=\sum_{j} \varphi_{j}^{\xi} c_{j}(\xi)
$$


where we have set $\hat{u}^{\xi}=u^{\xi}+v^{\xi}, \xi \in \Xi_{\rho}$. Therefore we can formally write

$$
\left.u_{t}\right|_{u=\hat{u}^{\xi}}=\sum_{j=1}^{N} c_{j}(\xi) \varphi_{j}^{\xi} .
$$

This indicates that $c(\xi)$ should play an important role in characterizing the dynamics of (1.17) near $\mathcal{M}$ and, since maps near $\mathcal{M}$ have a layered structure, $c(\xi)$ should be related to layers dynamics. Our first result is based on the fact that (1.17) is the $L^{2}$-gradient system associated to the action functional

$$
J_{\epsilon}(u)=\int_{0}^{1}\left(\epsilon^{2}\left|u_{x}\right|^{2}+W(u)\right) d x, u \in W^{1,2}\left((0,1) ; \mathbb{R}^{N}\right),
$$

and depends on the geometric structure of the graph of $J_{\epsilon}$ near $\mathcal{M}$. We decompose $u$ near $\mathcal{M}$ in the form (see Lemma 3.1)

$$
\begin{aligned}
& u=u^{\xi}+w, \\
& \left\langle w, u_{\xi_{j}}^{\xi}\right\rangle=0, \quad j=1, \ldots, N .
\end{aligned}
$$

By applying Theorem 2.1 in [11], we prove that, if the initial condition $u_{0}=u^{\xi_{0}}+w_{0}$ is sufficiently close to $\mathcal{M}$ and if the difference $J_{\epsilon}\left(u_{0}\right)-\sup _{\xi \in \Xi_{\rho}} J_{\epsilon}\left(u^{\xi}\right)$ is sufficiently small, then the solution $u\left(t, u_{0}\right)$ of (1.17) creeps along $\mathcal{M}$ for a very long time. We use the weighted $W^{1,2}$ norm

$$
\|v\|_{W_{\epsilon}^{1,2}}^{2}=\|v\|^{2}+\epsilon^{2}\left\|v_{x}\right\|^{2} .
$$

Theorem 1.2. Assume $\mathbf{H}_{\mathbf{1}}-\mathbf{H}_{\mathbf{3}}$ and assume that $u_{0}=u^{\xi_{0}}+w_{0}, \xi_{0} \in \Xi_{\rho}$, satisfies

$$
\begin{aligned}
& \left\langle w_{0}, u_{\xi_{j}}^{\xi_{0}}\right\rangle=0, \quad j=1, \ldots, N \\
& \left\|w_{0}\right\|_{W_{\epsilon}^{1,2}}=\mathrm{O}\left(\epsilon^{\frac{3}{2}}\right) \\
& \inf _{\zeta \in \partial \Xi_{\rho}}\left\|u^{\xi_{0}}-u^{\zeta}\right\|=d
\end{aligned}
$$

with $d>0$ independent of $\epsilon$.

(ii)

$$
J_{\epsilon}\left(u_{0}\right)-\sup _{\xi \in \Xi_{\rho}} J_{\epsilon}\left(u^{\xi}\right)=\mathrm{O}\left(e^{-\frac{\alpha \rho}{\epsilon}}\right) .
$$

Then there is $T>0$ such that the solution $t \rightarrow u\left(t, u_{0}\right)$ of (1.17) through $u_{0}$ can be decomposed in the form

$$
\begin{aligned}
& u\left(t, u_{0}\right)=u^{\xi(t)}+w(t), \quad t \in[0, T), \\
& \left\langle w(t), u_{\xi_{j}}^{\xi(t)}\right\rangle=0, \quad j=1, \ldots, N,
\end{aligned}
$$

and

$$
\|w(t)\|_{W_{\epsilon}^{1,2}}=\mathrm{O}\left(e^{-\frac{\alpha \rho}{\epsilon}}\right), \quad t \in[0, T) .
$$

Moreover either $T=+\infty$ and $\xi(t) \in \Xi_{\rho}$ for all $t>0$ or $T=\mathrm{O}\left(e^{\frac{\alpha \rho}{\epsilon}} d^{2}\right)$ and $\xi(T) \in \partial \Xi_{\rho}$. 
Based on the exponential estimate for $w(t)$ in Theorem 1.2 and on some ideas from [18] we prove that indeed $c(\xi)$ determines layers dynamics. As before we let $u\left(t, u_{0}\right)=$ $u^{\xi(t)}+w(t),\left\langle w(t), u_{\xi_{j}}^{\xi(t)}\right\rangle=0, \quad j=1, \ldots, N, t \in[0, T), T$ as in Theorem 1.2, be the decomposition of the solution $u\left(t, u_{0}\right)$ of (1.17) with initial condition $u_{0}=u^{\xi_{0}}+w_{0}$.

Theorem 1.3. Assume $\mathbf{H}_{1}-\mathbf{H}_{3}$. Then there exist $\epsilon_{0}>0$ and constants $Q>0, \beta>1$ independent of $\xi \in \Xi_{\rho}$ and $\epsilon \in\left(0, \epsilon_{0}\right]$ and such that, if $u_{0}$ is sufficiently close to $\mathcal{M}$ in the sense that

$$
\left\|w_{0}\right\|_{W_{\epsilon}^{1,2}} \leq Q\left\|\mathscr{F}\left(u^{\xi_{0}}\right)\right\|,
$$

Then, for each $t \in[0, T), T>0$ as in Theorem 1.2, we have $u\left(t, u_{0}\right)=u^{\xi(t)}+w(t)$ and

$$
\begin{aligned}
& \dot{\xi}_{j}=\frac{\epsilon^{\frac{1}{2}}}{\bar{q}_{j}} c_{j}(\xi)+\mathrm{O}\left(e^{-\frac{\alpha \rho}{\epsilon}} \max _{h, \pm} \mathscr{E}_{h}^{ \pm}\right), \quad j=1, \ldots, N, \\
& \|w(t)\|_{W_{\epsilon}^{1,2}} \leq \beta Q\left\|\mathscr{F}\left(u^{\xi(t)}\right)\right\| .
\end{aligned}
$$

From Theorem 1.3 and the explicit expression of $c(\xi)$ derived in Theorem 2.5 we have that the evolution of the layer positions $\xi_{1}, \ldots, \xi_{N}$ is determined by the equations

$$
\dot{\xi}_{j}=\frac{2 \epsilon}{\bar{q}_{j}^{2}}\left(\varsigma_{j+1} k_{j+1}^{+} \mathscr{E}_{j+1}-\varsigma_{j} k_{j}^{-} \mathscr{E}_{j}\right)+\mathrm{O}\left(e^{-\frac{\alpha \rho}{\epsilon}} \max _{h, \pm} \mathscr{E}_{h}^{ \pm}\right), \quad j=1, \ldots, N
$$

where $\varsigma_{j}=z_{j}^{-} \cdot z_{j}^{+}, \mu_{j}=\frac{1}{2}\left(\mu_{j}^{-}+\mu_{j}^{+}\right), k_{j}^{ \pm}=\mu_{j}^{ \pm} \mu_{j} \bar{K}_{j}^{-} \bar{K}_{j-1}^{+}$and

$$
\mathscr{E}_{j}=e^{-\frac{\mu_{j}}{\epsilon}\left(\xi_{j}-\xi_{j-1}\right)}, \quad \mathscr{E}_{j}^{ \pm}=e^{-\frac{\mu_{j}^{ \pm}}{\epsilon}\left(\xi_{j}-\xi_{j-1}\right)} .
$$

If $\mathbf{H}_{4}$ holds we have $z_{j}^{-}= \pm z_{j}^{+}, \mu_{j}=\mu_{j}^{-}=\mu_{j}^{+}, k_{j}^{ \pm}=k_{j}=\mu_{j}^{2} \bar{K}_{j}^{-} \bar{K}_{j-1}^{+}, \mathscr{E}_{j}=\mathscr{E}_{j}^{ \pm}$and (1.21) becomes

$$
\dot{\xi}_{j}=\frac{2 \epsilon}{\bar{q}_{j}^{2}}\left(\varsigma_{j+1} k_{j+1} \mathscr{E}_{j+1}-\varsigma_{j} k_{j} \mathscr{E}_{j}\right)+\mathrm{O}\left(e^{-\frac{\alpha \rho}{\epsilon}} \max _{h} \mathscr{E}_{h}\right), \quad j=1, \ldots, N
$$

with $\varsigma_{j}= \pm 1$. Equations (1.21) and (1.22) show that, as was conjectured in [14], beside their relevance in relation to the existence of periodic orbits of (1.9) (or equivalently stationary solutions of (1.17)) the scalar products $\varsigma_{j}=z_{j}^{-} \cdot z_{j}^{+}, j=1, \ldots, N$ have also a central importance in layers dynamics. Indeed (1.21) shows that, depending on the value of $\varsigma_{j}$ the term $\varsigma_{j} k_{j}^{-} \mathscr{E}_{j}$ can give a positive, negative or zero contribution to the speed $\dot{\xi}_{j}$ of the $j$-th layer. The value of the products $\varsigma_{j}$ also determines if consecutive layers attract or repel each other. To see this consider (1.22) where $\varsigma_{j}$ can only assume the extreme values \pm 1 and suppose that $\mu_{j}\left(\xi_{j}-\xi_{j-1}\right)>\mu_{h}\left(\xi_{h}-\xi_{h-1}\right)$ for $h \neq j$; then (1.22), for small $\epsilon>0$, imply

$$
\dot{\xi}_{j}-\dot{\xi}_{j-1}=-2 \varsigma_{j} k_{j} \mathscr{E}_{j}+\ldots
$$

where ... denotes higher order terms in $\epsilon$. From equation (1.23) we have attraction if $\varsigma_{j}=1$ and repulsion if $\varsigma_{j}=-1$. 

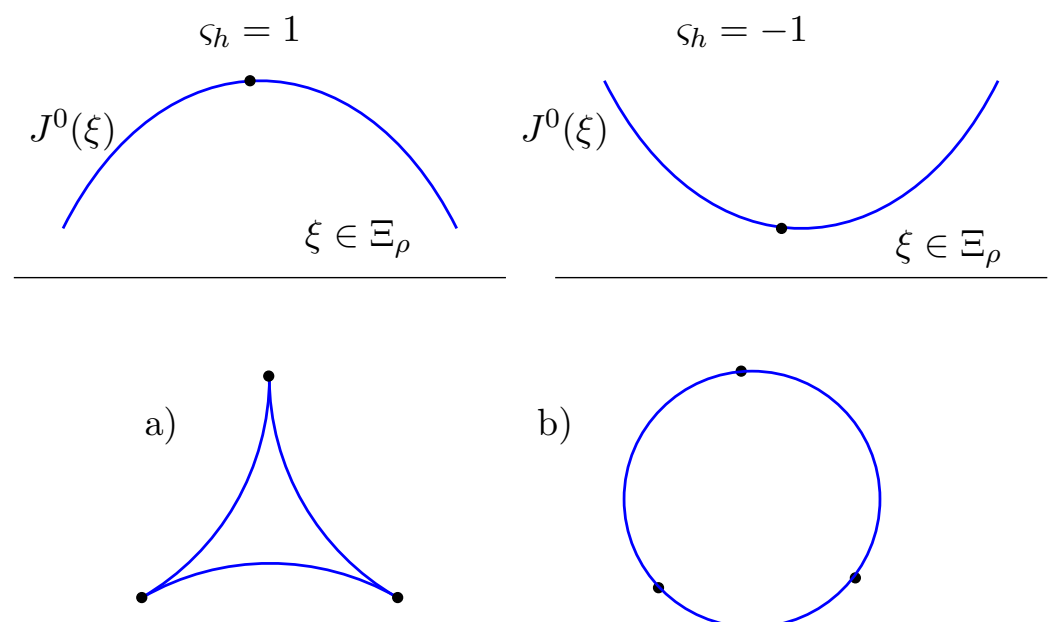

b)

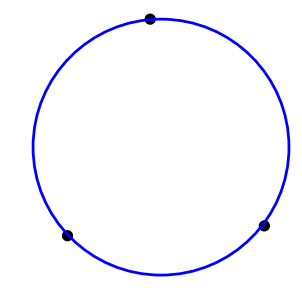

Figure 3: A potential with three zeros, $\varsigma_{h}= \pm 1$.

Remark. as we have observed, (1.17) is the $L^{2}$ gradient system associated to the functional (1.18). Therefore it is to be expected that (1.21) or (1.22) is approximately the gradient system of a suitable energy $J^{0}: \Xi_{\rho} \rightarrow \mathbb{R}$. We consider only the case of (1.22) and note that, indeed, if we set $C_{j}^{0}(\xi)=\frac{2 \epsilon}{\bar{q}_{j}^{2}}\left(\varsigma_{j+1} k_{j+1} \mathscr{E}_{j+1}-\varsigma_{j} k_{j} \mathscr{E}_{j}\right)$, we have

$$
\begin{aligned}
& \sum_{h} C_{h}^{0} \bar{q}_{h}^{2} \xi_{h}^{\prime}=-\sum \frac{\partial}{\partial \xi_{h}} J^{0}(\xi) \xi_{h}^{\prime}, \quad \xi^{\prime} \in \mathbb{R}^{N}, \\
& J^{0}(\xi)=\epsilon \sum_{j} \bar{q}_{j}^{2}-2 \epsilon^{2} \sum_{h} \frac{\varsigma_{h} k_{h}}{\mu_{h}} \mathscr{E}_{h},
\end{aligned}
$$

and therefore $\left(C_{1}^{0}, \ldots, C_{N}^{0}\right)$ is the negative of the gradient of $J^{0}$ with respect to the inner product $\left(\xi, \xi^{\prime}\right)=\sum_{h} \bar{q}_{h}^{2} \xi_{h} \xi_{h}^{\prime}$ which can be regarded as the restriction to the tangent space of $\mathcal{M}$ of the standard inner product in $L^{2}\left((0,1) ; \mathbb{R}^{m}\right)$. Note also that $J^{0}(\xi) \approx J_{\epsilon}\left(u^{\xi}\right)$, thus $J^{0}$ is approximately the restriction of $J_{\epsilon}(u)$ to $\mathcal{M}$.

In Fig. 3 we consider a potential $W \geq 0$ with three connected zeros. In case a) we have $\varsigma_{j}=1$ and in case b) $\varsigma_{j}=-1$. Under assumptions $\mathbf{H}_{1}-\mathbf{H}_{4}$ in case a) Theorem 1.1 gives the existence of a number of stationary solutions of (1.17) that diverges to $\infty$ as $\epsilon \rightarrow 0$ and $\varsigma_{j}=1$ implies that layers attract each other and is to be expected that all these stationary solutions are unstable for the dynamics of (1.17). In case b) from Theorem 1.1 we deduce the existence of just one stationary solution which, since $\varsigma_{j}=-1$ implies that layers repel each other, is conjectured to be stable. In Fig. 3 we also sketch the expected behavior of $J^{0}$ in a neighborhood of the stationary solution.

There is an extensive literature on layers dynamics, slow motion and related problems. Layers dynamics in the scalar case and for potentials with two minima, the typical example being $W(u)=\frac{1}{4}\left(1-u^{2}\right)^{2}$, was first described by Neu and then analyzed in [18, [24], 16], [19], 22], [37, [33] and [13] for the Allen-Cahn equation and [3], [17, [10], [12] for the Cahn-Hilliard equation. Slow motion of layers also appears in the context of dissipative hyperbolic equations [27], [21]. For the dynamics of the scalar Allen-Cahn equation with general nonlinearity we refer to [28], [29] and the references there in. For the vector case $m>1$ we refer to [25, 14] and [15]. The phenomenon of slow motion in higher dimension was studied in [8], [5], [7, 38, 20] and [31. 


\section{The proof of Theorem 1.1}

The analysis of equation (1.15) is based on the estimate (1.12) (see Lemma 2.7) and on the properties of the linear operator $L^{\xi}: W^{2,2}\left((0,1) ; \mathbb{R}^{m}\right) \rightarrow L^{2}\left((0,1) ; \mathbb{R}^{m}\right)$, acting on the subset of 1-periodic maps,

$$
L^{\xi} v=-\epsilon^{2} v_{x x}+W_{u u}\left(u^{\xi}\right) v
$$

that we collect in the following proposition

Proposition 2.1. Assume $\mathbf{H}_{1}-\mathbf{H}_{3}$. There exist $\epsilon_{0}>0, \lambda^{*}>0$ and $\mu^{*}>0$ such that, for $\epsilon \in\left(0, \epsilon_{0}\right], \xi \in \Xi_{\rho}$, the operator $L^{\xi}$ has $N$ eigenvalues $\lambda_{1}^{\xi}, \ldots, \lambda_{N}^{\xi}$ that satisfy

$$
\left|\lambda_{j}^{\xi}\right| \leq C e^{-\frac{\alpha \rho}{\epsilon}}, j=1, \ldots, N .
$$

If $\lambda \neq \lambda_{1}^{\xi}, \ldots, \lambda_{N}^{\xi}$ is an eigenvalue of $L^{\xi}$ then

$$
\lambda \geq \lambda^{*} .
$$

Let $X^{\xi}$ the eigenspace associated to the spectral set $\left\{\lambda_{1}^{\xi}, \ldots, \lambda_{N}^{\xi}\right\}$, then $X^{\xi}$ has a hortonormal basis $\left\{\varphi_{1}^{\xi}, \ldots, \varphi_{N}^{\xi}\right\}$ of the form

$$
\varphi_{j}^{\xi}:=\frac{u_{\xi_{j}}^{\xi}}{\left\|u_{\xi_{j}}^{\xi}\right\|}+\eta_{j}^{\xi} \in X^{\xi},\left\|\eta_{j}^{\xi}\right\|=O\left(e^{-\frac{\alpha \rho}{\epsilon}}\right) .
$$

Let $X^{\xi, \perp}$ the orthogonal complement of $X^{\xi}:=\operatorname{Span}\left(\varphi_{1}^{\xi}, \ldots, \varphi_{N}^{\xi}\right)$. Then

$$
\begin{aligned}
& \left|\left\langle L^{\xi} v, v\right\rangle\right| \geq \lambda^{*}\|v\|^{2}, \quad v \in X^{\xi, \perp} \cap W^{1,2}\left((0,1) ; \mathbb{R}^{m}\right), \\
& \left|\left\langle L^{\xi} v, v\right\rangle\right| \geq \mu^{*}\|v\|_{W_{\epsilon}^{1,2}}^{2}, \quad v \in X^{\xi, \perp} \cap W^{1,2}\left((0,1) ; \mathbb{R}^{m}\right) .
\end{aligned}
$$

For the proof of Proposition 2.1] we refer to [11] Appendix A where the proof is done for the case of Neumann boundary conditions. The argument applies to the present case of periodic boundary conditions with slight modifications.

From Proposition $2.1 L^{\xi}$ is almost singular on $X^{\xi}$ and therefore equation (1.14), as it stands, can not be solved for $v$. Therefore we proceed as sketched before and begin by solving for $v \in X^{\xi, \perp}$ the projection (1.15) of equation (1.14) on $X^{\xi, \perp}$.

Given $v \in X^{\xi, \perp}$, by forming the inner product of (1.15) with $\varphi_{j}^{\xi}, j=1, \ldots, N$, and observing that $\left\langle L^{\xi} v, \varphi_{j}^{\xi}\right\rangle=0$, we obtain that $c(\xi, v)$ is uniquely determined by

$$
c_{j}(\xi, v)=\left\langle\mathscr{F}\left(u^{\xi}\right)-N^{\xi}(v), \varphi_{j}^{\xi}\right\rangle, \quad j=1, \ldots, N
$$

where we have set

$$
\begin{aligned}
& N^{\xi}(v):=W_{u}\left(u^{\xi}+v\right)-W_{u}\left(u^{\xi}\right)-W_{u u}\left(u^{\xi}\right) v, \\
& \mathscr{F}\left(u^{\xi}\right):=\epsilon^{2} u_{x x}^{\xi}-W_{u}\left(u^{\xi}\right),
\end{aligned}
$$

Let $V^{\xi}$ be the set of 1-periodic maps defined by

$$
V^{\xi}:=\left\{v \in X^{\xi, \perp} \cap W^{1,2}\left((0,1) ; \mathbb{R}^{m}\right):\|v\|_{W_{\epsilon}^{1,2}} \leq \frac{2}{\mu^{*}}\left\|\mathscr{F}\left(u^{\xi}\right)\right\|\right\},
$$

where $\mu^{*}$ is the constant in (2.5)).

We show that (1.15) with $c(\xi, v)$ given by (2.6) has a unique solution $v \in V^{\xi}$. 
Proposition 2.2. Assume $\mathbf{H}_{1}-\mathbf{H}_{3}$. There is $\epsilon_{0}>0$ such that, $\epsilon \in\left(0, \epsilon_{0}\right]$ implies the following: for each $\xi \in \Xi_{\rho}$ there exist uniquely determined $v^{\xi} \in V^{\xi}$ and $c(\xi)=c\left(\xi, v^{\xi}\right) \in$ $\mathbb{R}^{N}$ that solve (1.15). The maps $\Xi_{\rho} \ni \xi \rightarrow c(\xi) \in \mathbb{R}^{N}$ and $\Xi_{\rho} \ni \xi \rightarrow v^{\xi} \in W^{1,2}$ are smooth and

$$
\begin{aligned}
& \left\|v^{\xi}\right\|_{W_{\epsilon}^{1,2}} \leq \frac{2}{\mu^{*}}\left\|\mathscr{F}\left(u^{\xi}\right)\right\|, \\
& c(\xi)=\left(\left\langle\mathscr{F}\left(u^{\xi}\right), \varphi_{1}^{\xi}\right\rangle, \ldots,\left\langle\mathscr{F}\left(u^{\xi}\right), \varphi_{N}^{\xi}\right\rangle\right)^{\top}+O\left(\epsilon^{-\frac{1}{2}}\left\|\mathscr{F}\left(u^{\xi}\right)\right\|^{2}\right), \\
& \epsilon^{2}\left(u^{\xi}+v^{\xi}\right)_{x x}-W_{u}\left(u^{\xi}+v^{\xi}\right)=\sum_{j=1}^{N} c_{j}(\xi) \varphi_{j}^{\xi} .
\end{aligned}
$$

Proof. 1. As we have observed, given $v \in V^{\xi}$, (2.6) determines the unique $c(\xi, v)$ ensuring that the right hand side of

$$
-L^{\xi} \hat{v}=\sum_{j} \varphi_{j}^{\xi} c_{j}(\xi, v)+N^{\xi}(v)-\mathscr{F}\left(u^{\xi}\right)
$$

belongs to $X^{\xi, \perp}$. Moreover the smoothness of $u^{\xi}$ and $\varphi_{j}^{\xi}$ implies that the right hand side of (2.10) is a $W^{1,2}$ map. Therefore Proposition 2.1) implies that (2.10) has a unique solution $\hat{v} \in X^{\xi, \perp} \cap W^{1,2}\left((0,1) ; \mathbb{R}^{m}\right)$.

2. For $v \in V^{\xi}$ we have

$$
\|v\|_{L^{\infty}\left(\mathbb{R} ; \mathbb{R}^{m}\right)} \leq C_{1} \epsilon^{-\frac{1}{2}}\left\|\mathscr{F}\left(u^{\xi}\right)\right\|
$$

and

$$
\left\|N^{\xi}(v)\right\| \leq C_{2} \epsilon^{-\frac{1}{2}}\left\|\mathscr{F}\left(u^{\xi}\right)\right\|^{2} .
$$

Fix $\bar{x} \in[0,1]$ such that $|v(\bar{x})|=\min _{x \in[0,1]}|v(x)|$. Then we have

$$
|v(x)|^{2} \leq|v(\bar{x})|^{2}+2 \int_{\bar{x}}^{\bar{x}+1}\left|v(s)\left\|v^{\prime}(s) \mid d s \leq\right\| v\left\|^{2}+2\right\| v\|\| v^{\prime}\left\|\leq \frac{2}{\epsilon}\right\| v \|_{W_{\epsilon}^{1,2}}^{2},\right.
$$

and (2.11) follows from (2.8).

To prove (2.12) we note that, for each $x \in[0,1]$, it results

$$
N^{\xi}(v)=\int_{0}^{1}\left(W_{u u}\left(u^{\xi}+s v\right)-W_{u u}\left(u^{\xi}\right)\right) v d s=\int_{0}^{1} s \int_{0}^{1} W_{u u u}\left(u^{\xi}+r s v\right) v \cdot v d r d s .
$$

From this, since (1.12) implies

$$
\max _{|z| \leq C_{1} \epsilon^{-\frac{1}{2}}\left\|\mathscr{F}\left(u^{\xi}\right)\right\|}\left|W_{\text {uuu }}\left(u^{\xi}+z\right)\right| \leq C,
$$

on the basis of (2.11) we have,

$$
\left|N^{\xi}(v)\right| \leq C|v|^{2} \leq C \epsilon^{-\frac{1}{2}}\left\|\mathscr{F}\left(u^{\xi}\right)\right\||v|, \quad x \in[0,1],
$$

and (2.12) follows.

3. $\hat{v} \in V^{\xi}$. By taking the inner product of (2.10) with $\hat{v}$ we obtain via (2.5)

$$
\begin{aligned}
& \mu^{*}\|\hat{v}\|_{W_{\epsilon}^{1,2}}^{2} \leq\left\|N^{\xi}(v)\right\|\|\hat{v}\|+\left\|\mathscr{F}\left(u^{\xi}\right)\right\|\|\hat{v}\| \\
& \leq\left(C_{2} \epsilon^{-\frac{1}{2}}\left\|\mathscr{F}\left(u^{\xi}\right)\right\|+1\right)\left\|\mathscr{F}\left(u^{\xi}\right)\right\|\|\hat{v}\| .
\end{aligned}
$$


This and $\left\|\mathscr{F}\left(u^{\xi}\right)\right\|=O\left(e^{-\frac{\alpha \rho}{\epsilon}}\right)$ imply that, provided $\epsilon>0$ is sufficiently small, we have

$$
\|\hat{v}\|_{W_{\epsilon}^{1,2}} \leq \frac{2}{\mu^{*}}\left\|\mathscr{F}\left(u^{\xi}\right)\right\|
$$

that proves the claim.

4. The map $V^{\xi} \ni v \rightarrow \hat{v} \in V^{\xi}$ constructed in 3. is a contraction. By subtracting equation (2.10) written for $v, w \in V^{\xi}$ and taking the inner product with $\hat{v}-\hat{w}$ we get

$$
-\left\langle L^{\xi}(\hat{v}-\hat{w}), \hat{v}-\hat{w}\right\rangle=\left\langle N^{\xi}(v)-N^{\xi}(w), \hat{v}-\hat{w}\right\rangle .
$$

where we have used $\left\langle\varphi_{j}^{\xi}, \hat{v}-\hat{w}\right\rangle=0$. From (2.5) and (2.14) it follows

$$
\mu^{*}\|\hat{v}-\hat{w}\|_{W_{\epsilon}^{1,2}} \leq\left\|N^{\xi}(v)-N^{\xi}(w)\right\| .
$$

We have

$$
\begin{aligned}
& N^{\xi}(v)-N^{\xi}(w)=\int_{0}^{1}\left(W_{u u}\left(u^{\xi}+w+\tau(v-w)\right)-W_{u u}\left(u^{\xi}\right)\right)(v-w) d \tau \\
& =\int_{0}^{1} \int_{0}^{1} W_{u u u}\left(u^{\xi}+t(w+\tau(v-w))\right)(w+\tau(v-w))(v-w) d t d \tau,
\end{aligned}
$$

and therefore, since $v, w \in V^{\xi}$, by (2.11)

$$
\begin{gathered}
\left|N^{\xi}(v)-N^{\xi}(w)\right| \leq C(|v|+2|w|)|v-w| \leq 3 \frac{C}{\epsilon^{\frac{1}{2}}}\left\|\mathscr{F}\left(u^{\xi}\right)\right\||v-w|, \\
\Rightarrow \quad\left\|N^{\xi}(v)-N^{\xi}(w)\right\| \leq 3 \frac{C}{\epsilon^{\frac{1}{2}}}\left\|\mathscr{F}\left(u^{\xi}\right)\right\|\|v-w\|, \quad v, w \in V^{\xi} .
\end{gathered}
$$

From this and (2.15) we obtain

$$
\|\hat{v}-\hat{w}\|_{W_{\epsilon}^{1,2}} \leq 3 \frac{C}{\mu^{*} \epsilon^{\frac{1}{2}}}\left\|\mathscr{F}\left(u^{\xi}\right)\right\|\|v-w\|
$$

that implies the claim for $\epsilon>0$ sufficiently small.

5. Let $v^{\xi} \in V^{\xi}$ the fixed point of the contraction map $V^{\xi} \ni v \rightarrow \hat{v} \in V^{\xi}$. Clearly $v^{\xi}$ satisfies $(2.9){ }_{1}$. The estimate for $c(\xi)$ follows from

$$
c(\xi)=c\left(\xi, v^{\xi}\right)=-\left(\left\langle N^{\xi}\left(v^{\xi}\right)-\mathscr{F}\left(u^{\xi}\right), \varphi_{1}^{\xi}\right\rangle, \ldots,\left\langle N^{\xi}\left(v^{\xi}\right)-\mathscr{F}\left(u^{\xi}\right), \varphi_{N}^{\xi}\right\rangle\right)^{\top}
$$

and $(2.9)_{1}$ and (2.12); (2.9) 3 is just a rewriting of (1.15) with $v=v^{\xi}$. The proof is complete.

Remark. The definition of $u^{\xi}$ implies that $u^{\xi+s \nu}=u^{\xi}(\cdot-s),\left(\nu=(1, \ldots, 1)^{\top}\right)$ this and (2.1) imply that we also have $\varphi_{j}^{\xi+s \nu}=\varphi_{j}^{\xi}(\cdot-s)$ and in turn $v^{\xi+s \nu}=v^{\xi}(\cdot-s)$. These observations and (2.9) imply

$$
c(\xi+s \nu)=c(\xi),
$$

That is $c(\xi)$ depends on $\xi$ only through the differences $\xi_{h}-\xi_{h-1}, i=1, \ldots, N$ and the identity

$$
\sum_{h=1}^{N} \xi_{h}-\xi_{h-1}=\xi_{N}-\xi_{0}=1, \quad\left(\xi_{0}=\xi_{N}-1\right),
$$

implies that $c(\xi)$ is actually a function of $N-1$ variables. 
From Proposition 2.2 there is a periodic solution of (1.9) in a neighborhood of $u^{\xi}$ of size $\left\|\mathscr{F}\left(u^{\xi}\right)\right\|$ if and only if $\xi$ is a solution of the bifurcation equation

$$
c(\xi)=0 .
$$

From the above remark this is a system of $N$ equations in $N-1$ unknowns. The following Proposition shows that, in spite of this, it is possible to solve (2.17).

Proposition 2.3. For $\epsilon \in\left(0, \epsilon_{0}\right]$, for some $\epsilon_{0}>0$, and for each $\xi \in \Xi_{\rho}$ there exist numbers $p_{j}^{\xi} \in \mathbb{R}, j=1, \ldots, N$ that satisfy

$$
\begin{aligned}
& \sum_{j=1}^{N}\left(\bar{q}_{j}+p_{j}^{\xi}\right) c_{j}(\xi)=0, \\
& p_{j}^{\xi}=\mathrm{O}\left(e^{-\frac{\alpha \rho}{\epsilon}}\right),
\end{aligned}
$$

where $\bar{q}_{j}^{2}=\int_{\mathbb{R}}\left|\bar{u}_{j}^{\prime}\right|^{2} d s$.

Proof. Forming the inner product of $(2.9)_{3}$ with $u_{x}^{\xi}+v_{x}^{\xi}$ yields

$$
0=\int_{0}^{1} \frac{d}{d x}\left(\frac{\epsilon^{2}}{2}\left|u_{x}^{\xi}+v_{x}^{\xi}\right|^{2}-W\left(u^{\xi}+v^{\xi}\right)\right) d x=\sum_{j} c_{j}(\xi)\left\langle\varphi_{j}^{\xi}, u_{x}^{\xi}+v_{x}^{\xi}\right\rangle,
$$

where we have observed that the left hand side vanishes being the integral extended to one period of the derivative with respect to $x$ of a periodic function. Now we note that (1.11) implies $u_{x}^{\xi}=-\sum_{j} u_{\xi_{j}}^{\xi}$ and rewrite (2.19) in the form

$$
\begin{aligned}
0 & =\sum_{j} c_{j}(\xi)\left\langle\varphi_{j}^{\xi}, u_{x}^{\xi}+v_{x}^{\xi}\right\rangle=-\sum_{j} c_{j}(\xi)\left(\left\langle\varphi_{j}^{\xi}, \sum_{i} u_{\xi_{i}}^{\xi}\right\rangle-\left\langle\varphi_{j}^{\xi}, v_{x}^{\xi}\right\rangle\right) \\
& =-\sum_{j} c_{j}(\xi)\left(\left\langle\varphi_{j}^{\xi}, \sum_{i}\left\|u_{\xi_{i}}^{\xi}\right\|\left(\frac{u_{\xi_{i}}^{\xi}}{\left\|u_{\xi_{i}}^{\xi}\right\|}+\eta_{i}^{\xi}\right)\right\rangle-\left\langle\varphi_{j}^{\xi}, v_{x}^{\xi}+\sum_{i}\left\|u_{\xi_{i}}^{\xi}\right\| \eta_{i}^{\xi}\right\rangle\right) \\
& =-\sum_{j} c_{j}(\xi)\left(\left\langle\varphi_{j}^{\xi}, \sum_{i}\left\|u_{\xi_{i}}^{\xi}\right\| \varphi_{i}^{\xi}\right\rangle+\mathrm{O}\left(e^{-\frac{\alpha \rho}{\epsilon}}\right)\right) \\
& =-\sum_{j} c_{j}(\xi)\left(\left\|u_{\xi_{j}}^{\xi}\right\|+\mathrm{O}\left(e^{-\frac{\alpha \rho}{\epsilon}}\right)\right),
\end{aligned}
$$

Where we have used (2.4) and $\left\|v_{x}^{\xi}\right\|=\mathrm{O}\left(e^{-\frac{\alpha \rho}{\epsilon}}\right)$ that follows from (1.12) and Proposition 2.2. To conclude the proof we note that, see (2.33)

$$
\left\|u_{\xi_{j}}^{\xi}\right\|=\frac{1}{\epsilon^{\frac{1}{2}}} \bar{q}_{j}+\mathrm{O}\left(e^{-\frac{\alpha \rho}{\epsilon}}\right) .
$$

The proof of Theorem 1.1 is based on the detailed analysis of $c(\xi)$ in Theorem 2.5 and also utilizes assumption $\mathbf{H}_{4}$. Therefore is worthwhile to observe that, if all the components of $c(\xi)$ were equal or $c(\xi)$ were a scalar quantity, Proposition 2.3 would automatically imply $c(\xi)=0$ without any further analysis and without requiring $\mathbf{H}_{4}$. A nontrivial situation 
where $c(\xi)=0$ can be deduced directly from Proposition 2.3 arises when $W: \mathbb{R}^{2} \rightarrow \mathbb{R}$ is invariant under the rotation group $C_{N}$ of the regular polygon with $N \geq 3$ sides

$$
W(\omega u)=W(u), \quad u \in \mathbb{R}^{2}, \omega=\left(\begin{array}{ll}
\cos \frac{2 \pi}{N} & -\sin \frac{2 \pi}{N} \\
\sin \frac{2 \pi}{N} & \cos \frac{2 \pi}{N}
\end{array}\right) .
$$

We assume that $W \geq 0$ has exactly $N$ zeros $\omega^{j-1} a, j=1, \ldots, N$ for some nondegenerate $a \in \mathbb{R}^{2} \backslash\{0\}$. These assumptions imply the existence of a minimizer $\bar{u}$ of $J(u)$ in the class of maps that connect $a$ to $\omega a$. From Proposition 2.2 and Proposition 2.3 we have the following result on the existence of periodic solutions which are equivariant under $C_{N}$.

Theorem 2.4. Assume that $W: \mathbb{R}^{2} \rightarrow \mathbb{R}, W \geq 0$, satisfies (2.20) and that $\{W=0\}=$ $\left\{a, \omega a, \ldots, \omega^{N-1} a\right\}$ for some nondegenerate $a \in \mathbb{R}^{2} \backslash\{0\}$. Assume that the zero eigenvalue of the operator $L v=-v^{\prime \prime}+W_{u u}(\bar{u}) v$ is simple. Then there exist $T_{0}>0$ and a family $u^{T}$, $T \geq T_{0}$ of $T$-periodic solutions of (1.1) that satisfy

$$
u^{T}\left(t+\frac{T}{N}\right)=\omega u^{T}(t), \quad t \in \mathbb{R} .
$$

Moreover there are positive constants $k_{0}, K_{0}$ such that

$$
\begin{aligned}
& \left\|u^{T}-u^{T, \xi}\right\|_{W^{1,2}\left([0,1] ; \mathbb{R}^{2}\right)} \leq K_{0} e^{-k_{0} T} e^{-\frac{\mu_{m}}{2 N} T}, \\
& \mid J_{(0, T)}\left(u^{T}\right)-J_{(0, T)}\left(u^{T, \xi} \mid \leq K_{0} e^{-k_{0} T} e^{-\frac{\mu_{m}}{N} T},\right. \\
& J_{(0, T)}\left(u^{T, \xi}\right)=N \bar{q}^{2}+\mathrm{O}\left(e^{-\frac{\mu_{m}}{2 N} T}\right),
\end{aligned}
$$

where $\mu_{m}=\min \left\{\mu^{-}, \mu^{+}\right\}, \bar{q}^{2}=\int_{\mathbb{R}}\left|\bar{u}^{\prime}\right|^{2} d s$ and

$$
\begin{aligned}
& u^{T, \xi}(t)=\sum_{n<0} \sum_{h=1}^{N} \omega^{h-1}\left(\bar{u}\left(t-\left(n+\xi+\frac{h-1}{N}\right) T\right)-a\right)+a \\
& +\sum_{n \geq 0} \sum_{h=1}^{N} \omega^{h-1}\left(\bar{u}\left(t-\left(n+\xi+\frac{h-1}{N}\right) T\right)-\omega a\right), \quad \xi \in\left[0, \frac{1}{N}\right] .
\end{aligned}
$$

Proof. Set $u^{\xi}(\cdot)=u^{\frac{1}{\epsilon}, \xi}(\dot{\bar{\epsilon}})$. Since $\xi$ is a real parameter we find that the operator $L^{\xi} v=$ $-\epsilon^{2} v_{x x}+W_{u u}\left(u^{\xi}\right) v$, restricted to the class of 1 -periodic equivariant maps

$$
v\left(x+\frac{1}{N}\right)=\omega v(x),
$$

has a unique small eigenvalue $\lambda^{\xi}=\mathrm{O}\left(e^{-\frac{\alpha \rho}{\epsilon}}\right)$ with corresponding eigenvector $\varphi^{\xi}=\frac{u_{\xi}^{\xi}}{\left\|u_{\xi}^{\xi}\right\|}+$ $\mathrm{O}\left(e^{-\frac{\alpha \rho}{\epsilon}}\right)$. Then arguing as in Proposition 2.2 we have that, for each $\xi$ there is $c(\xi) \in \mathbb{R}$ and a 1-periodic equivariant map $v^{\xi}$ such that

$$
\begin{aligned}
& \epsilon^{2}\left(u^{\xi}+v^{\xi}\right)_{x x}-W_{u}\left(u^{\xi}+v^{\xi}\right)=c(\xi) \varphi^{\xi}, \\
& \left\langle v^{\xi}, \varphi^{\xi}\right\rangle=0 .
\end{aligned}
$$

The reasoning of Proposition 2.3 then shows that $c(\xi)=0$ and renders a 1-periodic equivariant solution $\hat{u}^{\xi}=u^{\xi}+v^{\xi}$ of (1.9). The change of variable $x=\frac{t}{T}, \epsilon=\frac{1}{T}$, yields the sought $T$-periodic equivariant solution $u^{T}$ of (1.1). The estimate (2.21) 3 follows from (2.34) and $J_{(0, T)}\left(u^{T, \xi}\right)=\frac{1}{\epsilon} J_{\epsilon}\left(u^{\xi}\right)$. The other two estimates (2.21) follow from (2.9). 


\subsection{Discussion of the bifurcation equation}

In this subsection we derive an explicit expression for $c(\xi)$ which is basic for the proof of Theorem 1.1 and for the description of layers dynamics.

We define $\bar{u}_{j}$ also for $j=N+1, s<0$ and for $j=0$ and $s>0$ by setting

$$
\begin{aligned}
& \bar{u}_{N+1}(s)-a_{N+1}=\bar{u}_{1}(s)-a_{1}, \quad s<0 \\
& \bar{u}_{0}(s)-a_{1}=\bar{u}_{N}(s)-a_{N+1}, \quad s>0 .
\end{aligned}
$$

We also set

$$
\xi_{0}=\xi_{N}-1, \quad \xi_{N+1}=\xi_{1}+1
$$

These definitions imply

$$
\begin{array}{lc}
z_{N+1}^{-}=z_{1}^{-}, & z_{1}^{+}=z_{N+1}^{+}, \\
\mu_{N+1}^{-}=\mu_{1}^{-}, & \mu_{1}^{+}=\mu_{N+1}^{+}, \\
\bar{K}_{N+1}^{-}=\bar{K}_{1}^{-}, & \bar{K}_{0}^{+}=\bar{K}_{N}^{+},
\end{array}
$$

We introduce the notation:

$$
\begin{aligned}
& \mu_{h}=\frac{1}{2}\left(\mu_{h}^{-}+\mu_{h}^{+}\right), \quad h=1, \ldots, N+1, \\
& k_{h}^{ \pm}=\mu_{h}^{ \pm} \mu_{h} \bar{K}_{h}^{-} \bar{K}_{h-1}^{+}, \quad h=1, \ldots, N+1, \\
& \varsigma_{h}=z_{h}^{-} \cdot z_{h}^{+}, \quad h=1, \ldots, N+1,
\end{aligned}
$$

and

$$
\begin{aligned}
& \mathscr{E}_{h}^{ \pm}=e^{-\frac{\mu_{h}^{ \pm}}{\epsilon}\left(\xi_{h}-\xi_{h-1}\right)}, \quad h=1, \ldots, N+1, \\
& \mathscr{E}_{h}=e^{-\frac{\mu_{h}}{\epsilon}\left(\xi_{h}-\xi_{h-1}\right)}, \quad h=1, \ldots, N+1 .
\end{aligned}
$$

Observe that we have $\xi_{1}-\xi_{0}=\xi_{N+1}-\xi_{N}$ and therefore $\mathscr{E}_{N+1}^{ \pm}=\mathscr{E}_{1}^{ \pm}$. This and the identity $1=\sum_{h}\left(\xi_{h}-\xi_{h-1}\right)$ imply that $\mathscr{E}_{1}^{ \pm}, \ldots, \mathscr{E}_{N}^{ \pm}$are not independent and one of them is determined by the values of the remaining $N-1$.

Note that (2.22) imply

$$
\varsigma_{N+1}=\varsigma_{1}, \quad k_{N+1}^{ \pm}=k_{1}^{ \pm} .
$$

If $\mathbf{H}_{4}$ holds $\left(z_{h}^{-}= \pm z_{h}^{+}\right)$we have

$$
\begin{aligned}
& \varsigma_{h}= \pm 1, \\
& \mathscr{E}_{h}^{ \pm}=\mathscr{E}_{h}, \\
& k_{h}^{ \pm}=k_{h}:=\mu_{h}^{2} \bar{K}_{h}^{-} \bar{K}_{h-1}^{+} .
\end{aligned}
$$

We prove

Theorem 2.5. There is $\epsilon_{0}>0$ such that for $\epsilon \in\left(0, \epsilon_{0}\right]$ and $\xi \in \Xi_{\rho}$ it results

$$
c(\xi)=c^{0}(\xi)+r(\xi),
$$

where

$$
\begin{aligned}
c_{j}^{0}(\xi) & =\frac{2 \epsilon^{\frac{1}{2}}}{\bar{q}_{j}}\left(\varsigma_{j+1} k_{j+1}^{+} \mathscr{E}_{j+1}-\varsigma_{j} k_{j}^{-\mathscr{E}_{j}}\right), \quad j=1, \ldots, N, \\
r_{j}(\xi) & =\mathrm{O}\left(e^{-\frac{\alpha \rho}{\epsilon}} \max _{h, \pm} \mathscr{E}_{h}^{ \pm}\right) .
\end{aligned}
$$


Note that if $\mathbf{H}_{4}$ holds we have $\mu_{h}^{-}=\mu_{h}^{+}, \mathscr{E}_{h}=\mathscr{E}_{h}^{ \pm}, k_{h}^{ \pm}=k_{h}=\mu_{h}^{2} \bar{K}_{h}^{-} \bar{K}_{h-1}^{+}$and therefore

$$
\begin{aligned}
& c_{j}^{0}(\xi)=\frac{2 \epsilon^{\frac{1}{2}}}{\bar{q}_{j}}\left(\varsigma_{j+1} k_{j+1} \mathscr{E}_{j+1}-\varsigma_{j} k_{j} \mathscr{E}_{j}\right), \\
& r_{j}(\xi)=\mathrm{O}\left(e^{-\frac{\alpha \rho}{\epsilon}} \max _{h} \mathscr{E}_{h}\right)
\end{aligned}
$$

with $\varsigma_{j}= \pm 1$.

Remark. From Theorem 2.5 $c_{j}^{0}(\xi)$ depends only on the differences $\xi_{j}-\xi_{j-1}$ and $\xi_{j+1}-\xi_{j}$ and we expect that, at least approximately, the same is true for $c_{j}(\xi)$. Actually this is not always the case. Indeed, depending on the values of the $\mu_{j}^{ \pm}$and on the spacing of $\xi_{h}$ the contribution of the term $\mathrm{O}\left(e^{-\frac{\alpha \rho}{\epsilon}} \max _{h, \pm} \mathscr{E}_{h}^{ \pm}\right)$may exceed $c_{j}^{0}(\xi)$ and therefore $c_{j}(\xi)$ may depend on $\xi_{h}$ for $h \notin\{j-1, j, j+1\}$. In spite of this, the estimate provided by Theorem 2.5 is sufficient for the proof of Theorem 1.1 and to derive information on layers dynamics. We also note that we don't expect that a better analysis of the difference $c_{j}(\xi)-c_{j}^{0}(\xi)$ is possible under our general assumptions. Improving the estimate of $c_{j}(\xi)-c_{j}^{0}(\xi)$ given by Theorem 2.5 requires specific assumptions on the values of $\mu_{h}^{ \pm}$and on the position of the $\xi_{h}$.

Proof. (Proof of Theorem 2.5) The proof of Theorem 2.5 is quite elaborate and requires several steps and lemmas.

We begin with a detailed analysis of the expression (1.11) of $u^{\xi}, \xi \in \Xi_{\rho}$. Given $1 \leq j \leq N$, using the identity

$$
a_{1}+\sum_{h \leq j-1}\left(\bar{u}_{h}\left(\frac{x-\xi_{h}}{\epsilon}\right)-a_{h}\right)=\sum_{h \leq j-1}\left(\bar{u}_{h}\left(\frac{x-\xi_{h}}{\epsilon}\right)-a_{h+1}\right)+a_{j},
$$

we rewrite (1.11) in the form

$$
u^{\xi}(x)=\bar{u}_{j-1}\left(\frac{x-\xi_{j-1}}{\epsilon}\right)-a_{j}+\bar{u}_{j}\left(\frac{x-\xi_{j}}{\epsilon}\right)+\bar{u}_{j+1}\left(\frac{x-\xi_{j+1}}{\epsilon}\right)-a_{j+1}+\tau_{j},
$$

where

$$
\begin{aligned}
& \tau_{j}=\sum_{h<j-1}\left(\bar{u}_{h}\left(\frac{x-\xi_{h}}{\epsilon}\right)-a_{h+1}\right)+\sum_{h>j+1}\left(\bar{u}_{h}\left(\frac{x-\xi_{h}}{\epsilon}\right)-a_{h}\right) \\
& +\sum_{h=1}^{N} \sum_{n<0}\left(\bar{u}_{h}\left(\frac{x-n-\xi_{h}}{\epsilon}\right)-a_{h+1}\right)+\sum_{h=1}^{N} \sum_{n>0}\left(\bar{u}_{h}\left(\frac{x-n-\xi_{h}}{\epsilon}\right)-a_{h}\right) .
\end{aligned}
$$

We also consider

$$
\begin{aligned}
& \epsilon u_{\xi_{j}}^{\xi}(x)=-\bar{u}_{j}^{\prime}\left(\frac{x-\xi_{j}}{\epsilon}\right)-\sum_{n \neq 0} \bar{u}_{j}^{\prime}\left(\frac{x-n-\xi_{j}}{\epsilon}\right)=-\bar{u}_{j}^{\prime}\left(\frac{x-\xi_{j}}{\epsilon}\right)+\varkappa_{j}, \\
& \varkappa_{j}=-\sum_{n \neq 0} \bar{u}_{j}^{\prime}\left(\frac{x-n-\xi_{j}}{\epsilon}\right) .
\end{aligned}
$$

Lemma 2.6. For small $\epsilon>0$ we have the estimates

$$
\begin{aligned}
& \left|\tau_{j}\right| \leq C e^{-\frac{\alpha \rho}{\epsilon}} \max _{h, \pm} \mathscr{E}_{h}^{ \pm}, x \in\left[\hat{\xi}_{j-1}, \hat{\xi}_{j}\right], \quad \hat{\xi}_{h}=\frac{1}{2}\left(\xi_{h+1}+\xi_{h}\right), \\
& \left|\varkappa_{j}\right| \leq C\left(\mathscr{E}_{j}^{-}+\mathscr{E}_{j+1}^{+}\right), \quad x \in[0,1] .
\end{aligned}
$$


Moreover $\left|\epsilon \tau_{j x}\right|,\left|\epsilon \tau_{j \xi_{j}}\right|,\left|\epsilon^{2} \tau_{j x x}\right|$ and $\left|\epsilon^{3} \tau_{j x x \xi_{j}}\right|$ are bounded in $\left[\hat{\xi}_{j-1}, \hat{\xi}_{j}\right]$ by $C e^{-\frac{\alpha \rho}{\epsilon}} \max _{h, \pm} \mathscr{E}_{h}^{ \pm}$. A similar statement applies to $\varkappa_{j}$ in $[0,1]$. We also have

$$
\begin{aligned}
& \left\|u_{\xi_{j}}^{\xi}\right\|=\frac{1}{\epsilon^{\frac{1}{2}}} \bar{q}_{j}+\mathrm{O}\left(\mathscr{E}_{j}^{-}+\mathscr{E}_{j+1}^{+}\right), \quad\left(\bar{q}_{j}^{2}=\int_{\mathbb{R}}\left|\bar{u}_{j}^{\prime}\right|^{2} d s\right), \\
& \left\|u_{\xi_{i} \xi_{j}}^{\xi}\right\| \leq \frac{C}{\epsilon^{\frac{3}{2}}} \delta_{i j}, \\
& \left\|u_{x}^{\xi}\right\| \leq \frac{C}{\epsilon^{\frac{1}{2}}}, \quad\left\|u_{x x}^{\xi}\right\| \leq \frac{C}{\epsilon^{\frac{3}{2}}},
\end{aligned}
$$

$$
J_{\epsilon}\left(u^{\xi}\right)=\epsilon \sum_{j} \bar{q}_{j}^{2}+\mathrm{O}\left(\epsilon \max _{h, \pm} \mathscr{E}_{h}^{ \pm \frac{1}{2}}\right)
$$

and

$$
\frac{\left|u_{\xi_{i}}^{\xi}(x) \cdot u_{\xi_{j}}^{\xi}(x)\right|}{\left\|u_{\xi_{i}}^{\xi}\right\|\left\|u_{\xi_{j}}^{\xi}\right\|} \leq \frac{C}{\epsilon} \max _{h, \pm} \mathscr{E}_{h}^{ \pm}, \quad i \neq j .
$$

Proof. See Section 2.2 .

Next we derive accurate estimates for $\mathscr{F}\left(u^{\xi}\right)=\epsilon^{2} u_{x x}^{\xi}-W_{u}\left(u^{\xi}\right)$ :

Lemma 2.7. We have

$$
\begin{aligned}
& \left|\mathscr{F}\left(u^{\xi}\right)\right| \leq C e^{-\frac{\alpha \rho}{\epsilon}} \max _{h, \pm} \mathscr{E}_{h}^{ \pm \frac{1}{2}}, \quad x \in[0,1], \\
& \left|\mathscr{F}_{\xi_{j}}\left(u^{\xi}\right)\right| \leq \frac{C}{\epsilon} \max _{h, \pm} \mathscr{E}_{h}^{ \pm}, \quad x \in[0,1] .
\end{aligned}
$$

Proof. 1. Set $U(x)=\bar{u}_{j-1}\left(\frac{x-\xi_{j-1}}{\epsilon}\right)-a_{j}+\bar{u}_{j+1}\left(\frac{x-\xi_{j+1}}{\epsilon}\right)-a_{j+1}$. From (1.8) we have

$$
\begin{aligned}
& x \geq \xi_{j-1} \Rightarrow \\
& \left|\bar{u}_{j-1}\left(\frac{x-\xi_{j-1}}{\epsilon}\right)-a_{j}\right| \leq C e^{-\frac{\mu_{j}^{+}}{\epsilon}\left(x-\xi_{j-1}\right)}, \\
& x \leq \xi_{j+1} \Rightarrow \\
& \left|\bar{u}_{j+1}\left(\frac{x-\xi_{j+1}}{\epsilon}\right)-a_{j+1}\right| \leq C e^{-\frac{\mu_{j+1}^{-}}{\epsilon}\left(\xi_{j+1}-x\right)} .
\end{aligned}
$$

It follows

$$
\begin{aligned}
& |U| \leq C\left(\mathscr{E}_{j}^{+\frac{1}{2}}+\mathscr{E}_{j+1}^{-\frac{1}{2}}\right), \quad x \in\left[\hat{\xi}_{j-1}, \hat{\xi}_{j}\right], \\
& |U|^{2} \leq C\left(\mathscr{E}_{j}^{+}+\mathscr{E}_{j+1}^{-}\right), \quad x \in\left[\hat{\xi}_{j-1}, \hat{\xi}_{j}\right]
\end{aligned}
$$

From (2.29) we have

$$
\begin{aligned}
& -\mathscr{F}\left(u^{\xi}\right)=W_{u}\left(\bar{u}_{j}\left(\frac{x-\xi_{j}}{\epsilon}\right)+U+\tau_{j}\right)-\bar{u}_{j}^{\prime \prime}\left(\frac{x-\xi_{j}}{\epsilon}\right)-\epsilon^{2} U_{x x}-\epsilon^{2} \tau_{j x x} \\
& =W_{u}\left(\bar{u}_{j}\left(\frac{x-\xi_{j}}{\epsilon}\right)+U\right)-W_{u}\left(\bar{u}_{j}\left(\frac{x-\xi_{j}}{\epsilon}\right)\right)-\epsilon^{2} U_{x x}+\tau_{j}^{1} \quad x \in\left[\hat{\xi}_{j-1}, \hat{\xi}_{j}\right] .
\end{aligned}
$$


where we have used $\bar{u}_{j}^{\prime \prime}=W_{u}\left(\bar{u}_{j}\right)$ and denoted $\tau_{j}^{1}$ a quantity that satisfies (2.32). We have

$$
\begin{aligned}
& -\mathscr{F}\left(u^{\xi}\right)=\int_{0}^{1} W_{u u}\left(\bar{u}_{j}\left(\frac{x-\xi_{j}}{\epsilon}\right)+s U\right) U d s-\epsilon^{2} U_{x x}+\tau_{j}^{1} \\
& =W_{u u}\left(\bar{u}_{j}\left(\frac{x-\xi_{j}}{\epsilon}\right)\right) U-\epsilon^{2} U_{x x}+\mathrm{O}\left(|U|^{2}\right)+\tau_{j}^{1}, \quad x \in\left[\hat{\xi}_{j-1}, \hat{\xi}_{j}\right],
\end{aligned}
$$

and using (2.38)

$$
-\mathscr{F}\left(u^{\xi}\right)=W_{u u}\left(\bar{u}_{j}\left(\frac{x-\xi_{j}}{\epsilon}\right)\right) U-\epsilon^{2} U_{x x}+\mathrm{O}\left(\mathscr{E}_{j}^{+}+\mathscr{E}_{j+1}^{-}\right)+\tau_{j}^{1}, \quad x \in\left[\hat{\xi}_{j-1}, \hat{\xi}_{j}\right] .
$$

We now continue the analysis of $\mathscr{F}\left(u^{\xi}\right)$ separately in the intervals $\left[\hat{\xi}_{j-1}, \xi_{j}\right]$ and $\left[\xi_{j}, \hat{\xi}_{j}\right]$. We observe that, since $\mu_{h}^{ \pm 2}$ is the eigenvalue of $W_{u u}\left(a_{h}\right)$ associated to the eigenvector $z_{h}^{ \pm}$, (1.8) implies

$$
\begin{aligned}
& \bar{u}_{h}^{\prime \prime}(s)=W_{u u}\left(a_{h}\right)\left(\bar{u}_{h}(s)-a_{h}\right)+\mathrm{O}\left(e^{\hat{\mu}_{h}^{-} s}\right), \quad s \leq 0, \\
& \bar{u}_{h-1}^{\prime \prime}(s)=W_{u u}\left(a_{h}\right)\left(\bar{u}_{h-1}(s)-a_{h}\right)+\mathrm{O}\left(e^{-\hat{\mu}_{h-1}^{+} s}\right), \quad s \geq 0 .
\end{aligned}
$$

Writing simply $\bar{u}_{h}$ instead of $\bar{u}_{h}\left(\frac{x-\xi_{h}}{\epsilon}\right)$ we have

$$
\begin{aligned}
& W_{u u}\left(\bar{u}_{j}\right) U-\epsilon^{2} U_{x x}=W_{u u}\left(a_{j}\right) U-\epsilon^{2} U_{x x}+\text { Int } \\
& =W_{u u}\left(a_{j}\right)\left(\bar{u}_{j-1}-a_{j}\right)-\bar{u}_{j-1}^{\prime \prime}+W_{u u}\left(a_{j}\right)\left(\bar{u}_{j+1}-a_{j+1}\right)-\bar{u}_{j+1}^{\prime \prime}+\text { Int },
\end{aligned}
$$

where

$$
\begin{aligned}
& |\operatorname{Int}|=\left|\int_{0}^{1} W_{u u}\left(a_{j}+s\left(\bar{u}_{j}-a_{j}\right)\right)\left(\bar{u}_{j}-a_{j}\right) d s U\right| \\
& \leq C\left|\bar{u}_{j}-a_{j}\right||U| \leq C e^{\frac{\mu_{j}^{-}}{\epsilon}\left(x-\xi_{j}\right)}\left(e^{-\frac{\mu_{j}^{+}}{\epsilon}\left(x-\xi_{j-1}\right)}+e^{\frac{\mu_{j+1}^{-}}{\epsilon}\left(x-\xi_{j+1}\right)}\right) \\
& \leq C\left(\mathscr{E}_{j}+\mathscr{E}_{j}^{+}+\mathscr{E}_{j+1}^{-}\right), \quad x \in\left[\hat{\xi}_{j-1}, \xi_{j}\right] .
\end{aligned}
$$

where we have assumed $x \in\left[\hat{\xi}_{j-1}, \xi_{j}\right]$ and also used (2.37). From (2.42) and (1.8), we also have

$$
\begin{aligned}
& \left|W_{u u}\left(a_{j}\right)\left(\bar{u}_{j-1}-a_{j}\right)-\bar{u}_{j-1}^{\prime \prime}\right| \leq C e^{-\frac{\hat{\mu}_{j-1}^{+}}{\epsilon}\left(x-\xi_{j-1}\right)} \\
& \leq C e^{-\frac{\hat{\mu}_{j-1}^{+}}{2 \epsilon}\left(\xi_{j}-\xi_{j-1}\right)} \leq C e^{-\frac{\hat{\mu}_{j-1}^{+}-\mu_{j}^{+}}{2 \epsilon}\left(\xi_{j}-\xi_{j-1}\right)} \mathscr{E}_{j}^{+\frac{1}{2}}, \quad x \in\left[\hat{\xi}_{j-1}, \xi_{j}\right],
\end{aligned}
$$

and

$$
\begin{aligned}
& \left|W_{u u}\left(a_{j}\right)\left(\bar{u}_{j+1}-a_{j+1}\right)\right|+\left|\bar{u}_{j+1}^{\prime \prime}\right| \\
& \leq C e^{\frac{\mu_{j+1}^{-}}{\epsilon}\left(x-\xi_{j+1}\right)} \leq C \mathscr{E}_{j+1}^{-}, \quad x \in\left[\hat{\xi}_{j-1}, \xi_{j}\right] .
\end{aligned}
$$

These estimates and (2.43) yield

$$
\left|W_{u u}\left(\bar{u}_{j}\right) U-\epsilon^{2} U_{x x}\right| \leq C\left(e^{-\frac{\hat{\mu}_{j-1}^{+}-\mu_{j}^{+}}{2 \epsilon}}\left(\xi_{j}-\xi_{j-1}\right) \mathscr{E}_{j}^{+\frac{1}{2}}+\mathscr{E}_{j}+\mathscr{E}_{j}^{+}+\mathscr{E}_{j+1}^{-}\right), \quad x \in\left[\hat{\xi}_{j-1}, \xi_{j}\right],
$$


A similar computation gives

$$
\left|W_{u u}\left(\bar{u}_{j}\right) U-\epsilon^{2} U_{x x}\right| \leq C\left(e^{-\frac{\hat{\mu}_{j+1}^{-}-\mu_{j+1}^{-}}{2 \epsilon}\left(\xi_{j+1}-\xi_{j}\right)} \mathscr{E}_{j+1}^{-\frac{1}{2}}+\mathscr{E}_{j+1}+\mathscr{E}_{j}^{+}+\mathscr{E}_{j+1}^{-}\right), \quad x \in\left[\xi_{j}, \hat{\xi}_{j+1}\right] .
$$

From (2.41), (2.45) and (2.46) we finally conclude

$$
\left|\mathscr{F}\left(u^{\xi}\right)\right| \leq C e^{-\frac{\alpha \rho}{\epsilon}} \max _{h, \pm} \mathscr{E}_{h}^{ \pm \frac{1}{2}}+\left|\tau_{j}^{1}\right| \leq C e^{-\frac{\alpha \rho}{\epsilon}} \max _{h, \pm} \mathscr{E}_{h}^{ \pm \frac{1}{2}}, \quad x \in\left[\hat{\xi}_{j-1}, \hat{\xi}_{j}\right] .
$$

where we have used (2.32). Since this is valid for all $j(2.36) 1$ follows.

To estimate $\mathscr{F}_{\xi_{j}}\left(u^{\xi}\right)$ we differentiate the expression of $\mathscr{F}\left(u^{\xi}\right)$ given in (2.39) and observe that $U_{\xi_{j}}=U_{x x \xi_{j}}=0$ to obtain

$$
-\mathscr{F}_{\xi_{j}}\left(u^{\xi}\right)=-\left(W_{u u}\left(\bar{u}_{j}+U+\tau_{j}\right)-W_{u u}\left(\bar{u}_{j}\right)\right) \frac{1}{\epsilon} \bar{u}_{j}^{\prime}+W_{u u}\left(\bar{u}_{j}+U+\tau_{j}\right) \tau_{j \xi_{j}}+\epsilon^{2} \tau_{j x x \xi_{j}},
$$

where we have used $\bar{u}_{j}^{\prime \prime \prime}=W_{u u}\left(\bar{u}_{j}\right) \bar{u}_{j}^{\prime}$. It follows

$$
\left|\mathscr{F}_{\xi_{j}}\left(u^{\xi}\right)\right| \leq \frac{C}{\epsilon}\left(\left(|U|+\left|\tau_{j}\right|\right)\left|\bar{u}_{j}^{\prime}\right|+\epsilon\left|\tau_{j \xi_{j}}\right|+\epsilon^{3}\left|\tau_{j x x \xi_{j}}\right|\right)
$$

As in the estimate (2.44) for Int, from (1.8) and (2.37) it follows

$$
\begin{aligned}
& |U|\left|\bar{u}_{j}^{\prime}\right| \leq C e^{\frac{\mu_{j}^{-}}{\epsilon}\left(x-\xi_{j}\right)}\left(e^{-\frac{\mu_{j}^{+}}{\epsilon}\left(x-\xi_{j-1}\right)}+e^{\frac{\mu_{j+1}^{-}}{\epsilon}\left(x-\xi_{j+1}\right)}\right) \\
& \leq C \max _{h, \pm} \mathscr{E}_{h}^{ \pm}, \quad x \in\left[\hat{\xi}_{j-1}, \xi_{j}\right], \\
& |U|\left|\bar{u}_{j}^{\prime}\right| \leq C e^{-\frac{\mu_{j}^{+}}{\epsilon}\left(x-\xi_{j}\right)}\left(e^{-\frac{\mu_{j}^{+}}{\epsilon}\left(x-\xi_{j-1}\right)}+e^{\frac{\mu_{j+1}^{-}}{\epsilon}\left(x-\xi_{j+1}\right)}\right) \\
& \leq C \max _{h, \pm} \mathscr{E}_{h}^{ \pm}, \quad x \in\left[\xi_{j}, \hat{\xi}_{j}\right] .
\end{aligned}
$$

From this and the estimates for $\tau_{j}, \epsilon \tau_{j \xi_{j}}$ and $\epsilon^{3} \tau_{j x x \xi_{j}}$ the bound for $\mathscr{F}_{\xi_{j}}\left(u^{\xi}\right)$ follows. The proof is complete

A first step in the analysis of $c(\xi)$ is an asymptotic formula for $\bar{c}(\xi)$ defined by

$$
\bar{c}_{j}(\xi)=\left\langle\mathscr{F}\left(u^{\xi}\right), \frac{u_{\xi_{j}}^{\xi}}{\left\|u_{\xi_{j}}^{\xi}\right\|}\right\rangle, \quad j=1, \ldots, N .
$$

Lemma 2.8. There is $\epsilon_{0}>$ such that, for $\epsilon \in\left(0, \epsilon_{0}\right]$, it results

$$
\bar{c}_{j}(\xi)=\frac{2 \epsilon^{\frac{1}{2}}}{\bar{q}_{j}}\left(\varsigma_{j+1} k_{j+1}^{+} \mathscr{E}_{j+1}-\varsigma_{j} k_{j}^{-} \mathscr{E}_{j}+\mathrm{O}\left(e^{-\frac{\alpha \rho}{\epsilon}} \max _{h, \pm} \mathscr{E}_{h}^{ \pm}\right)\right), \quad j=1, \ldots, N .
$$

where $\varsigma_{j}$ and $k_{j}^{ \pm}$are defined in (2.23) and $\bar{q}_{j}^{2}=\int_{\mathbb{R}}\left|\bar{u}_{j}^{\prime}\right|^{2} d s$.

Proof. In this proof, if there is no risk of confusion, we simply write $\bar{u}_{h}$ instead of $\bar{u}_{h}\left(\frac{x-\xi_{h}}{\epsilon}\right)$. From (2.40) and the expression of $U$ we obtain

$$
\begin{aligned}
& \bar{c}_{j}(\xi)=\frac{1}{\left\|\epsilon u_{\xi_{j}}^{\xi}\right\|}\left(\int_{0}^{1} \mathscr{F}\left(u^{\xi}\right) \cdot \varkappa_{j} d x-\int_{0}^{\hat{\xi}_{j-1}} \mathscr{F}\left(u^{\xi}\right) \cdot \bar{u}_{j}^{\prime} d x-\int_{\hat{\xi}_{j}}^{1} \mathscr{F}\left(u^{\xi}\right) \cdot \bar{u}_{j}^{\prime} d x\right) \\
& +\frac{1}{\left\|\epsilon u_{\xi_{j}}^{\xi}\right\|} \int_{\hat{\xi}_{j-1}}^{\hat{\xi}_{j}}\left(W_{u u}\left(\bar{u}_{j}\right)\left(\bar{u}_{j-1}-a_{j}+\bar{u}_{j+1}-a_{j+1}\right)\right. \\
& \left.-\bar{u}_{j-1}^{\prime \prime}-\bar{u}_{j+1}^{\prime \prime}+\mathrm{O}\left(\left|\tau_{j}^{1}\right|+|U|^{2}\right)\right) \cdot \bar{u}_{j}^{\prime} d x
\end{aligned}
$$


From Lemma 2.6 and Lemma 2.7 we have

$$
\begin{aligned}
& \left|\mathscr{F}\left(u^{\xi}\right) \cdot \varkappa_{j}\right| \leq C e^{-\frac{\alpha \rho}{\epsilon}} \max _{h, \pm} \mathscr{E}_{h}^{ \pm}, \quad x \in[0,1], \\
& \left|\mathscr{F}\left(u^{\xi}\right) \cdot \bar{u}_{j}^{\prime}\right| \leq C\left|\mathscr{F}\left(u^{\xi}\right)\right| e^{-\frac{\mu_{j}^{-}}{2 \epsilon}\left(\xi_{j}-\xi_{j-1}\right)} \leq C e^{-\frac{\alpha \rho}{\epsilon}} \max _{h, \pm} \mathscr{E}_{h}^{ \pm}, \quad x \leq \hat{\xi}_{j-1}, \\
& \left|\mathscr{F}\left(u^{\xi}\right) \cdot \bar{u}_{j}^{\prime}\right| \leq C\left|\mathscr{F}\left(u^{\xi}\right)\right| e^{-\frac{\mu_{j+1}^{+}}{2 \epsilon}\left(\xi_{j+1}-\xi_{j}\right)} \leq C e^{-\frac{\alpha \rho}{\epsilon}} \max _{h, \pm} \mathscr{E}_{h}^{ \pm}, \quad x \geq \hat{\xi}_{j}, \\
& \left|\tau_{j}^{1}\right|\left|\bar{u}_{j}^{\prime}\right| \leq C e^{-\frac{\alpha \rho}{\epsilon}} \max _{h, \pm} \mathscr{E}_{h}^{ \pm}, \quad x \in\left[\hat{\xi}_{j-1}, \hat{\xi}_{j}\right] .
\end{aligned}
$$

and by mean of (2.37) we find

$$
\begin{aligned}
& |U|^{2}\left|\bar{u}_{j}^{\prime}\right| \leq 2\left(\left|\bar{u}_{j-1}-a_{j}\right|^{2}+\left|\bar{u}_{j+1}-a_{j+1}\right|^{2}\right)\left|\bar{u}_{j}^{\prime}\right| \\
& \leq C\left(e^{-\frac{2 \mu_{j}^{+}}{\epsilon}\left(x-\xi_{j-1}\right)}+e^{\frac{2 \mu_{j+1}^{-}}{\epsilon}\left(x-\xi_{j+1}\right)}\right) e^{\frac{\mu_{j}^{-}}{\epsilon}\left(x-\xi_{j}\right)} \\
& \leq C e^{-\frac{\alpha \rho}{\epsilon}} \max _{h, \pm} \mathscr{E}_{h}^{ \pm}, \quad x \in\left[\hat{\xi}_{j-1}, \xi_{j}\right],
\end{aligned}
$$

and in a similar way

$$
|U|^{2}\left|\bar{u}_{j}^{\prime}\right| \leq C e^{-\frac{\alpha \rho}{\epsilon}} \max _{h, \pm} \mathscr{E}_{h}^{ \pm}, \quad x \in\left[\xi_{j}, \hat{\xi}_{j}\right] .
$$

These estimates imply that we can rewrite (2.48) in the form

$$
\begin{aligned}
& \bar{c}_{j}(\xi)=\frac{1}{\left\|\epsilon u_{\xi_{j}}^{\xi}\right\|}\left(\int_{\hat{\xi}_{j-1}}^{\hat{\xi}_{j}}\left(W_{u u}\left(\bar{u}_{j}\right)\left(\bar{u}_{j-1}-a_{j}+\bar{u}_{j+1}-a_{j+1}\right)-\bar{u}_{j-1}^{\prime \prime}-\bar{u}_{j+1}^{\prime \prime}\right) \cdot \bar{u}_{j}^{\prime} d x\right. \\
& +\mathrm{O}\left(e^{-\frac{\alpha \rho}{\epsilon}} \max _{h, \pm} \mathscr{E}_{h}^{ \pm}\right)=\frac{1}{\left\|\epsilon u_{\xi_{j}}^{\xi}\right\|}\left(I+\mathrm{O}\left(e^{-\frac{\alpha \rho}{\epsilon}} \max _{h, \pm} \mathscr{E}_{h}^{ \pm}\right)\right),
\end{aligned}
$$

with obvious definition of $I$. Since $W_{u u}(u)$ is a symmetric matrix and $\bar{u}_{j}^{\prime \prime \prime}=W_{u u}\left(\bar{u}_{j}\right) \bar{u}_{j}^{\prime}$, we can rewrite $I$ as

$$
\begin{aligned}
I & =\int_{\hat{\xi}_{j-1}}^{\hat{\xi}_{j}}\left(W_{u u}\left(\bar{u}_{j}\right) \bar{u}_{j}^{\prime} \cdot\left(\bar{u}_{j-1}-a_{j}+\bar{u}_{j+1}-a_{j+1}\right)-\left(\bar{u}_{j-1}^{\prime \prime}+\bar{u}_{j+1}^{\prime \prime}\right) \cdot \bar{u}_{j}^{\prime}\right) d x \\
& =\int_{\hat{\xi}_{j-1}}^{\hat{\xi}_{j}}\left(\bar{u}_{j}^{\prime \prime \prime} \cdot\left(\bar{u}_{j-1}-a_{j}+\bar{u}_{j+1}-a_{j+1}\right)-\left(\bar{u}_{j-1}^{\prime \prime}+\bar{u}_{j+1}^{\prime \prime}\right) \cdot \bar{u}_{j}^{\prime}\right) d x .
\end{aligned}
$$

Set

$$
g=\bar{u}_{j}^{\prime \prime} \cdot\left(\bar{u}_{j-1}-a_{j}+\bar{u}_{j+1}-a_{j+1}\right)-\bar{u}_{j}^{\prime} \cdot\left(\bar{u}_{j-1}^{\prime}+\bar{u}_{j+1}^{\prime}\right) .
$$

We regard $g$ as a function of $s=\frac{x}{\epsilon}$ and we have $\epsilon \frac{d}{d x} g=g^{\prime}$. Note that

$$
g^{\prime}=\bar{u}_{j}^{\prime \prime \prime} \cdot\left(\bar{u}_{j-1}-a_{j}+\bar{u}_{j+1}-a_{j+1}\right)-\left(\bar{u}_{j-1}^{\prime \prime}+\bar{u}_{j+1}^{\prime \prime}\right) \cdot \bar{u}_{j}^{\prime},
$$

that is $g^{\prime}$ coincides with the integrand of $I$. It follows

$$
I=\int_{\hat{\xi}_{j-1}}^{\hat{\xi}_{j}} \epsilon \frac{d}{d x} g d x=\left.\epsilon g\right|_{\hat{\xi}_{j-1}} ^{\hat{\xi}_{j}} .
$$

From (1.8) we obtain 


$$
\begin{aligned}
& \left.\bar{u}_{j}^{\prime \prime}\right|_{x=\hat{\xi}_{j}}=z_{j+1}^{+} \mu_{j+1}^{+}{ }^{2} \bar{K}_{j}^{+} e^{-\frac{\mu_{j+1}^{+}}{2 \epsilon}\left(\xi_{j+1}-\xi_{j}\right)}+\mathrm{O}\left(e^{-\frac{\hat{\mu}_{j}^{+}-\mu_{j+1}^{+}}{2 \epsilon}\left(\xi_{j+1}-\xi_{j}\right)} e^{-\frac{\mu_{j+1}^{+}}{2 \epsilon}\left(\xi_{j+1}-\xi_{j}\right)}\right) \\
& =z_{j+1}^{+} \mu_{j+1}^{+}{ }^{2} \bar{K}_{j}^{+} \mathscr{E}_{j+1}^{+}{ }^{\frac{1}{2}}+\mathrm{O}\left(e^{-\frac{\alpha \rho}{\epsilon}} \mathscr{E}_{j+1}^{+} \frac{1}{2}\right) \\
& \left.\left(\bar{u}_{j+1}-a_{j+1}\right)\right|_{x=\hat{\xi}_{j}}=z_{j+1}^{-} \bar{K}_{j+1}^{-} e^{-\frac{\mu_{j+1}^{-}}{2 \epsilon}\left(\xi_{j+1}-\xi_{j}\right)}+\mathrm{O}\left(e^{-\frac{\hat{\mu}_{j+1}^{-}-\mu_{j+1}^{-}}{2 \epsilon}\left(\xi_{j+1}-\xi_{j}\right)} e^{-\frac{\mu_{j+1}^{-}}{2 \epsilon}\left(\xi_{j+1}-\xi_{j}\right)}\right) \\
& =z_{j+1}^{-} \bar{K}_{j+1}^{-} \mathscr{E}_{j+1}^{-\frac{1}{2}}+\mathrm{O}\left(e^{-\frac{\alpha \rho}{\epsilon}} \mathscr{E}_{j+1}^{-\frac{1}{2}}\right) \\
& -\left.\bar{u}_{j}^{\prime}\right|_{x=\hat{\xi}_{j}}=z_{j+1}^{+} \mu_{j+1}^{+} \bar{K}_{j}^{+} \mathscr{E}_{j+1}^{+}{ }^{\frac{1}{2}}+\mathrm{O}\left(e^{-\frac{\alpha \rho}{\epsilon}} \mathscr{E}_{j+1}^{+}{ }^{\frac{1}{2}}\right) \\
& \left.\bar{u}_{j+1}^{\prime}\right|_{x=\hat{\xi}_{j}}=z_{j+1}^{-} \mu_{j+1}^{-} \bar{K}_{j+1}^{-} \mathscr{E}_{j+1}^{\frac{1}{2}}+\mathrm{O}\left(e^{-\frac{\alpha \rho}{\epsilon}} \mathscr{E}_{j+1}^{\frac{1}{2}}\right) .
\end{aligned}
$$

Since from (2.24) $\mathscr{E}_{h}^{-\frac{1}{2}} \mathscr{E}_{h}^{+\frac{1}{2}}=\mathscr{E}_{h}$, it follows

$$
\begin{aligned}
& \left.\bar{u}_{j}^{\prime \prime} \cdot\left(\bar{u}_{j+1}-a_{j+1}\right)\right|_{x=\hat{\xi}_{j}}=\varsigma_{j+1} \mu_{j+1}^{+}{ }^{2} \bar{K}_{j+1}^{-} \bar{K}_{j}^{+} \mathscr{E}_{j+1}+\mathrm{O}\left(e^{-\frac{\alpha \rho}{\epsilon}} \mathscr{E}_{j+1}\right) \\
& -\left.\bar{u}_{j}^{\prime} \cdot \bar{u}_{j+1}^{\prime}\right|_{x=\hat{\xi}_{j}}=\varsigma_{j+1} \mu_{j+1}^{+} \mu_{j+1}^{-} \bar{K}_{j+1}^{-} \bar{K}_{j}^{+} \mathscr{E}_{j+1}+\mathrm{O}\left(e^{-\frac{\alpha \rho}{\epsilon}} \mathscr{E}_{j+1}\right),
\end{aligned}
$$

and recalling the definition (2.23) of $k_{j}^{ \pm}$we finally obtain

$$
\left.\left(\bar{u}_{j}^{\prime \prime} \cdot\left(\bar{u}_{j+1}-a_{j+1}\right)-\bar{u}_{j}^{\prime} \cdot \bar{u}_{j+1}^{\prime}\right)\right|_{x=\hat{\xi}_{j}}=\varsigma_{j+1} 2 k_{j+1}^{+} \mathscr{E}_{j+1}+\mathrm{O}\left(e^{-\frac{\alpha \rho}{\epsilon}} \mathscr{E}_{j+1}\right)
$$

A similar computation yields

$$
\left.\left(\bar{u}_{j}^{\prime \prime} \cdot\left(\bar{u}_{j-1}-a_{j}\right)-\bar{u}_{j}^{\prime} \cdot \bar{u}_{j-1}^{\prime}\right)\right|_{x=\hat{\xi}_{j-1}}=\varsigma_{j} 2 k_{j}^{-} \mathscr{E}_{j}+\mathrm{O}\left(e^{-\frac{\alpha \rho}{\epsilon} \mathscr{E}_{j}}\right)
$$

and we also have

$$
\begin{aligned}
& \left.\left(\bar{u}_{j}^{\prime \prime} \cdot\left(\bar{u}_{j+1}-a_{j+1}\right)-\bar{u}_{j}^{\prime} \cdot \bar{u}_{j+1}^{\prime}\right)\right|_{x=\hat{\xi}_{j-1}}=\mathrm{O}\left(e^{-\frac{\mu_{j}^{-}+\mu_{j+1}^{-}}{2 \epsilon}\left(\xi_{j}-\xi_{j-1}\right)} e^{-\frac{\mu_{j+1}^{-}}{\epsilon}\left(\xi_{j+1}-\xi_{j}\right)}\right) \\
& =\mathrm{O}\left(e^{-\frac{\alpha \rho}{\epsilon}} \mathscr{E}_{j+1}^{-}\right) \\
& \left.\left(\bar{u}_{j}^{\prime \prime} \cdot\left(\bar{u}_{j-1}-a_{j}\right)-\bar{u}_{j}^{\prime} \cdot \bar{u}_{j-1}^{\prime}\right)\right|_{x=\hat{\xi}_{j}}=\mathrm{O}\left(e^{-\frac{\mu_{j}^{+}+\mu_{j+1}^{+}}{2 \epsilon}\left(\xi_{j+1}-\xi_{j}\right)} e^{-\frac{\mu_{j}^{+}}{\epsilon}\left(\xi_{j}-\xi_{j-1}\right)}\right) \\
& =\mathrm{O}\left(e^{-\frac{\alpha \rho}{\epsilon}} \mathscr{E}_{j}^{+}\right)
\end{aligned}
$$

From these estimates (2.50) and (2.48) we get

$$
\bar{c}_{j}(\xi)=\frac{2}{\left\|u_{\xi_{j}}^{\xi}\right\|}\left(\varsigma_{j+1} k_{j+1} \mathscr{E}_{j+1}-\varsigma_{j} k_{j} \mathscr{E}_{j}+\mathrm{O}\left(e^{-\frac{\alpha \rho}{\epsilon}} \max _{h, \pm} \mathscr{E}_{h}^{ \pm}\right)\right), \quad j=1, \ldots, N .
$$

This and (2.33) complete the proof.

To obtain a good approximation to $c(\xi)$ valid for $\epsilon>0$ small, the estimate for $\eta_{j}^{\xi}$ in (2.4) is not sufficient. We need the following refinement, 
Lemma 2.9. Let $X^{\xi}$ the eigen-space introduced in Proposition 2.1. There exist vectors $\eta_{1}^{\xi}, \ldots, \eta_{N}^{\xi} \in W^{1,2}\left((0,1) ; \mathbb{R}^{m}\right)$ such that

(i) $\left\|\eta_{j}^{\xi}\right\| \leq \frac{C}{\epsilon} \max _{h, \pm} \mathscr{E}_{h}^{ \pm}$.

(ii) $\varphi_{j}^{\xi}=\frac{u_{\xi_{j}}^{\xi}}{\left\|u_{\xi_{j}}^{\xi}\right\|}+\eta_{j}^{\xi}, \quad j=1, \ldots, N$ is an orthonormal basis for $X^{\xi}$.

Proof. See Section 2.2

From (2.36) and Lemma 2.9 it follows

$$
\begin{aligned}
& \left\|\mathscr{F}\left(u^{\xi}\right)\right\|^{2} \leq C e^{-\frac{\alpha \rho}{\epsilon}} \max _{h, \pm} \mathscr{E}_{h}^{ \pm}, \\
& \left.\left|\left\langle\mathscr{F}\left(u^{\xi}\right), \eta_{j}^{\xi}\right\rangle\right| \leq C e^{-\frac{\alpha \rho}{\epsilon}} \max _{h, \pm} \mathscr{E}_{h}^{ \pm}\right) .
\end{aligned}
$$

This (2.9) and Lemma 2.8 yield $c_{j}(\xi)-\bar{c}_{j}(\xi)=\mathrm{O}\left(e^{-\frac{\alpha \rho}{\epsilon}} \max _{h, \pm} \mathscr{E}_{h}^{ \pm}\right)$and we can conclude that

$$
\begin{aligned}
c_{j}(\xi) & =c_{j}^{0}(\xi)+r_{j}(\xi) \\
r_{j}(\xi) & =\mathrm{O}\left(e^{-\frac{\alpha \rho}{\epsilon}} \max _{h, \pm} \mathscr{E}_{h}^{ \pm}\right), \quad j=1, \ldots, N .
\end{aligned}
$$

where $c_{j}^{0}(\xi)$ is defined in (2.28). This conclude the proof of Theorem 2.5 .

Note that, since, as we have observed, $c(\xi)$ depends on $\xi$ through the differences $\xi_{j}-\xi_{j-1}, j=1, \ldots, N$ and therefore is a function of only $N-1$ variables, equation (2.52) implies that also the error $r_{j}(\xi)$ in the estimate (2.52) depends only on $\xi_{j}-\xi_{j-1}$, $j=1, \ldots, N$.

We can now state

Theorem 2.10. Assume $\mathbf{H}_{\mathbf{1}}-\mathbf{H}_{\mathbf{4}}$, then there exists $\epsilon_{0}>0$ such that, provided $\epsilon \in\left(0, \epsilon_{0}\right]$, the condition

$$
\varsigma_{j}=\varsigma_{j+1}, \quad j=1, \ldots, N
$$

is necessary and sufficient in order that there is $\xi \in \Xi_{\rho}$ with the property that $\hat{u}^{\xi}=u^{\xi}+v^{\xi}$, $v^{\xi}$ as in Proposition 2.2, is a periodic solution of period 1 of (1.9). The vector $\xi \in \Xi_{\rho}$ that determines the periodic orbit satisfies

$$
\xi_{j}-\xi_{j-1}=\frac{1 / \mu_{j}}{\sum_{h=1}^{N} 1 / \mu_{h}}+\epsilon \delta_{j}(\xi), \quad j=1, \ldots, N, \quad \sum_{j=1}^{N} \delta_{j}=0 .
$$

where $\delta_{j}=\bar{\delta}_{j}+\mathrm{O}\left(e^{-\frac{\alpha \rho}{\epsilon}}\right)$ and $\bar{\delta}_{j}$ are constants that depend only on $\mu_{1}, \ldots, \mu_{N}$ and $k_{1}, \ldots, k_{N}$. Proof. From Theorem 2.5 we have $c(\xi)=0$ if and only if

$$
\varsigma_{j+1} k_{j+1} \mathscr{E}_{j+1}=\varsigma_{j} k_{j} \mathscr{E}_{j}+\frac{\bar{q}_{j} r_{j}(\xi)}{2 \epsilon^{\frac{1}{2}}}, \quad j=1, \ldots, N .
$$

Since we are assuming $\mathbf{H}_{4},(2.28)$ implies that at least one of the $\mathscr{E}_{j}$ coincides with $\max _{h} \mathscr{E}_{h}$ and that, for small $\epsilon>0, r_{j}(\xi)$ is much smaller than $\max _{h} \mathscr{E}_{h}$. From this and $\varsigma_{j}= \pm 1$ it 
follows that (2.53) is a necessary condition for the existence of a solution $\xi \in \Xi$ of (2.55) and it results

$$
C^{-} \max _{h} \mathscr{E}_{h} \leq \mathscr{E}_{j} \leq C^{+} \max _{h} \mathscr{E}_{h},
$$

for some constants $C^{ \pm}>0$ independent of $\epsilon$. This proves the necessity of condition (2.53). The prove the sufficiency we note that, with (2.53), equation (2.55) becomes

$$
k_{j+1} \mathscr{E}_{j+1}=k_{j} \mathscr{E}_{j}+\frac{\bar{q}_{j} \varsigma_{j} r_{j}(\xi)}{2 \epsilon^{\frac{1}{2}}}, j=1, \ldots, N .
$$

From Proposition 2.3 to show that there exists $\xi \in \Xi$ that solves the equation $c(\xi)=0$ it suffices to show that we can solve the first $N-1$ of the equations (2.57). These equations constitute a linear system in the unknowns $k_{j} \mathscr{E}_{j}, j=2, \ldots, N$ that can be solved yielding

$$
k_{j} \mathscr{E}_{j}=k_{1} \mathscr{E}_{1}+\frac{\varsigma_{1}}{2 \epsilon^{\frac{1}{2}}} \sum_{h=1}^{j-1} \bar{q}_{h} r_{h}(\xi), \quad j=2, \ldots, N
$$

If we take the logarithm of these equations and multiply by $-\epsilon$ we get

$$
\begin{aligned}
& \mu_{j}\left(\xi_{j}-\xi_{j-1}\right)=\mu_{1}\left(\xi_{j}-\xi_{0}\right)+\epsilon \ln \frac{k_{j}}{k_{1}}+\epsilon \tilde{r}_{j}(\xi), \quad j=2, \ldots, N . \\
& \tilde{r}_{j}(\xi)=-\ln \left(1+\frac{\varsigma_{1}}{2 \epsilon^{\frac{1}{2}} k_{1} \mathscr{E} 1} \sum_{h=1}^{j-1} \bar{q}_{h} r_{h}(\xi)\right) .
\end{aligned}
$$

We introduce the new variable $\delta \in \mathbb{R}^{N}$ by setting

$$
\begin{aligned}
& \xi_{j}-\xi_{j-1}=\frac{\frac{1}{\mu_{j}}}{\sum_{h} \frac{1}{\mu_{h}}}+\epsilon \delta_{j}, \quad j=1, \ldots, N, \\
& \sum_{j=1}^{N} \delta_{j}=0
\end{aligned}
$$

where $(2.601)_{2}$ follows from $\sum_{j=1}^{N}\left(\xi_{j}-\xi_{j-1}\right)=1$. Since, as $r(\xi), \tilde{r}(\xi)$ depends on $\xi$ through the differences $\xi_{j}-\xi_{j-1}, j=1, \ldots, N$ the function $\tilde{r}(\xi(\delta))$ is well defined via the change of variables (2.60). We also observe that, since $\rho>0$ in the definition (1.10) of $\Xi$ is a small fixed number, given $\delta_{0}>0$, for small $\epsilon>0$, we have

$$
\frac{\frac{1}{\mu_{j}}}{\sum_{h} \frac{1}{\mu_{h}}}+\epsilon \delta_{j}>\rho, \quad|\delta| \leq \delta_{0}, \quad j=1, \ldots, N,
$$

and therefore (2.28), (2.59) 2 and (2.56) imply

$$
\tilde{r}(\xi(\delta))=\mathrm{O}\left(e^{-\frac{\alpha \rho}{\epsilon}}\right), \quad|\delta| \leq \delta_{0} .
$$

With the new variable, after dividing by $\epsilon \mu_{j}$, (2.59) becomes

$$
\begin{aligned}
& \delta_{j}=\frac{\mu_{1}}{\mu_{j}} \delta_{1}+\frac{1}{\mu_{j}} \ln \frac{k_{j}}{k_{1}}+\frac{1}{\mu_{j}} \tilde{r}_{j}(\xi(\delta)), j=2, \ldots, N, \\
& \sum_{j=1}^{N} \delta_{j}=0 .
\end{aligned}
$$


We make a further change of variable by setting $\delta=\bar{\delta}+\delta^{*}$ where $\bar{\delta}$ is the solution of (2.63) corresponding to $\epsilon=0$ :

$$
\begin{aligned}
& \bar{\delta}_{1}=-\frac{1}{\mu_{1}} \frac{\sum_{h=2}^{N} \frac{1}{\mu_{h}} \ln \frac{k_{h}}{k_{1}}}{\sum_{h=1}^{N} \frac{1}{\mu_{h}}}, \\
& \bar{\delta}_{j}=\frac{\mu_{1}}{\mu_{j}} \bar{\delta}_{1}+\frac{1}{\mu_{j}} \ln \frac{k_{j}}{k_{1}}, j=2, \ldots, N .
\end{aligned}
$$

If we insert $\delta=\bar{\delta}+\delta^{*}$ in (2.63) we have

$$
\begin{aligned}
\delta_{j}^{*} & =\frac{1}{\mu_{j}} \tilde{r}_{j}\left(\xi\left(\bar{\delta}+\delta^{*}\right)\right), \quad j=2, \ldots, N, \\
\delta_{1}^{*} & =-\sum_{j=2}^{N} \delta_{j}^{*} .
\end{aligned}
$$

Set $B_{1}=\left\{\delta^{*} \in \mathbb{R}^{N}:\left|\delta^{*}\right| \leq 1, \sum_{h} \delta_{h}^{*}=0\right\}$ and let $F: B_{1} \rightarrow \mathbb{R}^{N}$ be defined by setting $F_{j}\left(\delta^{*}\right)=\tilde{r}_{j}\left(\xi\left(\bar{\delta}+\delta^{*}\right)\right), j=2, \ldots, N, F_{1}\left(\delta^{*}\right)=-\sum_{j=2}^{N} \tilde{r}_{j}\left(\xi\left(\bar{\delta}+\delta^{*}\right)\right) . \quad F$ is a continuous function that, by (2.62) satisfies $F\left(\delta^{*}\right)=\mathrm{O}\left(e^{-\frac{\alpha \rho}{\epsilon}}\right)$. It follows that $F$ is a continuous map that maps $B_{1}$ into itself and Brouwer fixed point theorem implies that $F$ has a fixed in $B_{1}$ that is a solution of (2.64) that we still denote $\delta^{*}$. Moreover from (2.62) it follows that $\delta^{*}=\mathrm{O}\left(e^{-\frac{\alpha \rho}{\epsilon}}\right)$. This concludes the proof.

Theorem 1.1 is essentially equivalent to Theorem 2.10. Theorem 2.10 shows that under the assumptions of Theorem 1.1 there exists a $\xi \in \Xi_{\rho}$ such that, for small $\epsilon>0, \hat{u}=u^{\xi}+v^{\xi}$, $v^{\xi}$ as in Proposition 2.2, is a 1-periodic solution of (1.9). If we set $\epsilon=\frac{1}{T}$ and $x=\frac{t}{T}$, then $u^{T}(t)=\hat{u}\left(\frac{t}{T}\right)$ is a $T$ - periodic solution of (1.1). From (2.29), (2.32), $\left\|v^{\xi}\right\|_{W_{\epsilon}^{1,2}} \leq C\left\|\mathscr{F}\left(u^{\xi}\right)\right\|$ and (2.36) it follows

$$
\begin{aligned}
& \left|\hat{u}(x)-\bar{u}_{j}\left(\frac{x-\xi_{j}}{\epsilon}\right)\right| \leq C\left(e^{-\frac{\mu_{j}}{\epsilon}\left(x-\xi_{j-1}\right)}+e^{\frac{\mu_{j+1}}{\epsilon}\left(x-\xi_{j+1}\right)}+e^{-\frac{\alpha \rho}{\epsilon}} \max _{h} \mathscr{E}_{h}^{\frac{1}{2}}\right) \\
& \left.\leq C e^{-\frac{1}{2 \epsilon \sum_{h} \frac{1}{\mu_{h}}}}\right), \quad x \in\left[\hat{\xi}_{j-1}, \hat{\xi}_{j}\right], j=1, \ldots, N,
\end{aligned}
$$

where we have also used the characterization (2.54) of $\xi$. In terms of $u^{T}(2.65)$ becomes

$$
u^{T}(t)=\bar{u}_{j}\left(t-\xi_{j} T\right)+\mathrm{O}\left(e^{-\frac{T}{2 \sum_{h} \frac{1}{\mu_{h}}}}\right), \quad t \in\left[\hat{\xi}_{j-1} T, \hat{\xi}_{j} T\right], j=1, \ldots, N,
$$

Statement (i) of Theorem 1.1 follows from (2.54) with $\epsilon=\frac{1}{T}$ and from (2.66) with the change of variable $t \rightarrow t+\xi_{j} T$. To prove (ii) we note that (1.8) implies the existence of a constant $C>0$ such that for small $\delta>0$ we have

$$
\begin{aligned}
& \left|\bar{u}_{j-1}-a_{j}\right| \leq \frac{\delta}{2}, \quad s \geq-C \ln \delta, \quad, \quad j=1, \ldots, N . \\
& \left|\bar{u}_{j}-a_{j}\right| \leq \frac{\delta}{2}, \quad s \leq C \ln \delta .
\end{aligned}
$$

This and (2.66) imply (ii) with $t_{\delta}=-C \ln \delta$. The last statement of Theorem 1.1 follows from $\left\|v^{\xi}\right\|_{W_{\epsilon}^{1,2}}=\mathrm{O}\left(e^{-\frac{\alpha \rho}{\epsilon}} e^{-\frac{1}{2 \epsilon \sum_{h} \frac{1}{\mu_{h}}}}\right)$. The proof of Theorem 1.1 is complete. 


\subsection{Proofs of Lemma 2.6 and Lemma 2.9.}

Proof. (Lemma 2.6) We assume $\left[\xi_{j-1}, \xi_{j+1}\right] \subset[0,1]$. The estimates that we derive are valid in any case.

We rearrange $\tau_{j}$ in the form

$$
\begin{aligned}
\tau_{j} & =\sum_{h<j-1} \sum_{n \leq 0}\left(\bar{u}_{h}\left(\frac{x-n-\xi_{h}}{\epsilon}\right)-a_{h+1}\right)+\sum_{h \geq j-1} \sum_{n<0}\left(\bar{u}_{h}\left(\frac{x-n-\xi_{h}}{\epsilon}\right)-a_{h+1}\right) \\
& +\sum_{h>j+1} \sum_{n \geq 0}\left(\bar{u}_{h}\left(\frac{x-n-\xi_{h}}{\epsilon}\right)-a_{h}\right)+\sum_{h \leq j+1} \sum_{n>0}\left(\bar{u}_{h}\left(\frac{x-n-\xi_{h}}{\epsilon}\right)-a_{h}\right) \\
& =\sum_{h<j-1} \tilde{\tau}_{h}^{+}+\sum_{h \geq j-1} \hat{\tau}_{h}^{+}+\sum_{h>j+1} \tilde{\tau}_{h}^{-}+\sum_{h \leq j+1} \hat{\tau}_{h}^{-},
\end{aligned}
$$

with obvious definition of $\tilde{\tau}_{h}^{ \pm}$and $\hat{\tau}_{h}^{ \pm}$. We only analyze the last two summations. The other two can be estimate in the same way.

1. Estimating $\sum_{h>j+1} \tilde{\tau}_{h}^{-}$.

Note that

$$
\begin{aligned}
& h>j+1 \text { and } x \leq \hat{\xi}_{j} \Rightarrow x-n-\xi_{h} \leq \frac{\xi_{j+1}+\xi_{j}}{2}-n-\xi_{h} \\
& =-\left(\frac{\xi_{j+1}-\xi_{j}}{2}+\xi_{h}-\xi_{j+1}+n\right) \leq-\left(\frac{\xi_{j+1}-\xi_{j}}{2}+\xi_{h}-\xi_{h-1}+n\right) .
\end{aligned}
$$

This and (1.8), observing also that the contribution of $\bar{w}_{h}^{-}$can be absorbed in the constant $C$, imply

$$
\begin{aligned}
& \left|\bar{u}_{h}\left(\frac{x-n-\xi_{h}}{\epsilon}\right)-a_{h}\right| \\
& \leq C e^{-\frac{\mu_{h}^{-}}{\epsilon} \frac{\xi_{j+1}-\xi_{j}}{2}} e^{-\frac{\mu_{h}^{-}}{\epsilon}\left(\xi_{h}-\xi_{h-1}\right)} e^{-\frac{\mu_{h}^{-}}{\epsilon} n}, \quad n=0,1, \ldots, x \leq \hat{\xi}_{j} .
\end{aligned}
$$

It follows

$$
\left|\tilde{\tau}_{h}^{-}\right| \leq C e^{-\frac{\mu_{h}^{-}}{\epsilon} \frac{\xi_{j+1}-\xi_{j}}{2}} \mathscr{E}_{h}^{-}, x \leq \hat{\xi}_{j}, h>j+1,
$$

where we have also used (2.24).

2. Estimating $\sum_{h \leq j+1} \hat{\tau}_{h}^{-}$. We have

$$
\begin{aligned}
& h \leq j+1 \text { and } x \leq \hat{\xi}_{j} \Rightarrow x-n-\xi_{h} \leq \frac{\xi_{j+1}+\xi_{j}}{2}-\xi_{h}-n \\
& =-\left(\frac{\xi_{j+1}-\xi_{j}}{2}+\xi_{h}-\xi_{j+1}+n\right) \leq-\left(\frac{\xi_{j+1}-\xi_{j}}{2}+\xi_{h}-\xi_{h-1}+(n-1)\right),
\end{aligned}
$$

where we have used that $h \leq j+1$ implies $\xi_{h}-\xi_{j+1}+1 \geq \xi_{h}-\xi_{h-1}$. This and (1.8) yield

$$
\left|\bar{u}_{h}\left(\frac{x-n-\xi_{h}}{\epsilon}\right)-a_{h}\right| \leq e^{-\frac{\mu_{h}^{-}}{\epsilon} \frac{\xi_{j+1}-\xi_{j}}{2}} e^{-\frac{\mu_{h}^{-}}{\epsilon}\left(\xi_{h}-\xi_{h-1}\right)} e^{-\frac{\mu_{h}^{-}}{\epsilon}(n-1)}, n>0 .
$$

It follows

$$
\left|\hat{\tau}_{h}^{-}\right| \leq C e^{-\frac{\mu_{h}^{-}}{\epsilon} \frac{\xi_{j+1}-\xi_{j}}{2}} \mathscr{E}_{h}^{-}, x \leq \hat{\xi}_{j}, h \leq j+1
$$


In the same way, since

$$
\begin{aligned}
& h<j-1, \quad n \leq 0, \text { and } x \geq \hat{\xi}_{j-1}, \Rightarrow \\
& x-n-\xi_{h} \geq \frac{1}{2}\left(\xi_{j}-\xi_{j-1}\right)+\xi_{j-1}-\xi_{h}+|n| \geq \frac{1}{2}\left(\xi_{j}-\xi_{j-1}\right)+\xi_{h+1}-\xi_{h}+|n|, \\
& h \geq j-1, \quad n<0, \text { and } x \geq \hat{\xi}_{j-1}, \Rightarrow \\
& x-n-\xi_{h} \geq \frac{1}{2}\left(\xi_{j}-\xi_{j-1}\right)+\xi_{j-1}-\xi_{h}+|n| \geq \frac{1}{2}\left(\xi_{j}-\xi_{j-1}\right)+1+\xi_{j-1}-\xi_{h}+(|n|-1) \\
& \geq \frac{1}{2}\left(\xi_{j}-\xi_{j-1}\right)+\xi_{h+1}-\xi_{h}+(|n|-1),
\end{aligned}
$$

we find

$$
\begin{aligned}
& \left|\tilde{\tau}_{h}^{+}\right| \leq C e^{-\frac{\mu_{h+1}^{+}}{\epsilon} \frac{\xi_{j}-\xi_{j-1}}{2}} \mathscr{E}_{h+1}^{+}, x \leq \hat{\xi}_{j}, h<j-1
\end{aligned}
$$

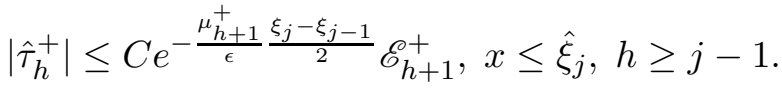

and we conclude that

$$
\begin{aligned}
& \left|\tau_{j}\right| \leq C \sum_{h}\left(e^{-\frac{\mu_{h}^{-}}{\epsilon} \frac{\xi_{j+1}-\xi_{j}}{2}} \mathscr{E}_{h}^{-}+e^{-\frac{\mu_{h+1}^{+}}{\epsilon} \frac{\xi_{j}-\xi_{j-1}}{2}} \mathscr{E}_{h+1}^{+}\right) \\
& \leq C e^{-\frac{\alpha \rho}{\epsilon}} \max _{h, \pm} \mathscr{E}_{h}^{ \pm} .
\end{aligned}
$$

This concludes the estimate of $\tau_{j}$.

3. Note that (1.8) and

$$
\begin{aligned}
& n>0 \text { and } x \in[0,1] \Rightarrow \\
& x-\xi_{j}-n \leq-\xi_{j}-(n-1) \leq-\left(\xi_{j}-\xi_{j-1}\right)-(n-1), \\
& n<0 \text { and } x \in[0,1] \Rightarrow \\
& x-\xi_{j}-n \geq-\xi_{j}+1+(|n|-1) \geq\left(\xi_{j+1}-\xi_{j}\right)+(|n|-1)
\end{aligned}
$$

imply

$$
\begin{aligned}
& \sum_{n>0}\left|\bar{u}_{j}^{\prime}\left(\frac{x-\xi_{j}-n}{\epsilon}\right)\right| \leq C e^{-\frac{\mu_{j}^{-}}{\epsilon}\left(\xi_{j}-\xi_{j-1}\right)}, \quad x \in[0,1], \\
& \sum_{n<0}\left|\bar{u}_{j}^{\prime}\left(\frac{x-\xi_{j}-n}{\epsilon}\right)\right| \leq C e^{-\frac{\mu_{j+1}^{+}}{\epsilon}\left(\xi_{j+1}-\xi_{j}\right)}, \quad x \in[0,1] .
\end{aligned}
$$

It follows

$$
\left|\varkappa_{j}\right| \leq C\left(\mathscr{E}_{j}^{-}+\mathscr{E}_{j+1}^{+}\right), \quad x \in[0,1] .
$$

From the expression (2.67) of $\tau_{j}$ we see that $\epsilon \tau_{j x}, \epsilon \tau_{j \xi_{j}}, \epsilon^{2} \tau_{j x x}$ and $\epsilon^{3} \tau_{j x x \xi_{j}}$ have a structure similar to $\tau_{j}$ and therefore, (1.8) implies that can be estimated by the same arguments developed for estimating $\tau_{j}$. The same remark applies to $\varkappa_{j}$.

4. From (2.31) we have

$$
\left\|\epsilon u_{\xi_{j}}^{\xi}\right\|=\left\|\bar{u}_{j}^{\prime}\left(\frac{\cdot-\xi_{j}}{\epsilon}\right)\right\|+\mathrm{O}\left(\left\|\varkappa_{j}\right\|\right)
$$


and recalling that $\bar{q}_{j}^{2}=\int_{\mathbb{R}}\left|\bar{u}_{j}^{\prime}\right|^{2} d s$

$$
\begin{aligned}
& \left|\left\|\bar{u}_{j}^{\prime}\left(\frac{-\xi_{j}}{\epsilon}\right)\right\|^{2}-\epsilon \bar{q}_{j}^{2}\right| \leq \epsilon \int_{-\infty}^{-\frac{\xi_{j}}{\epsilon}}\left|\bar{u}_{j}^{\prime}(s)\right|^{2} d s+\epsilon \int_{\frac{1-\xi_{j}}{\epsilon}}^{+\infty}\left|\bar{u}_{j}^{\prime}(s)\right|^{2} d s \\
& \leq C \epsilon\left(\mathscr{E}_{j}^{-2}+\mathscr{E}_{j+1}^{+}{ }^{2}\right),
\end{aligned}
$$

where we have also used (1.8) and the assumption $\left[\xi_{j-1}, \xi_{j+1}\right] \subset[0,1]$. This and the estimate for $\varkappa_{j}$ imply $(2.33)_{1}$. Similarly we have

$$
\begin{aligned}
& \left\|\epsilon^{2} u_{\xi_{j} \xi_{j}}^{\xi}\right\| \leq\left\|\bar{u}_{j}^{\prime \prime}\left(\frac{\cdot-\xi_{j}}{\epsilon}\right)\right\|+\left\|\epsilon^{2} \varkappa_{j \xi_{j} \xi_{j}}\right\|, \\
& \left\|\bar{u}_{j}^{\prime \prime}\left(\frac{\cdot-\xi_{j}}{\epsilon}\right)\right\|^{2} \leq \epsilon \int_{\mathbb{R}}\left|\bar{u}_{j}^{\prime \prime}(s)\right|^{2} d s
\end{aligned}
$$

that together with $u_{\xi_{i} \xi_{j}}^{\xi}=0$ for $i \neq j$ yield $(\underline{2.33})_{2}$. The proof of $(2.33)_{3}$ follows from the previous estimates for $u_{\xi_{j}}^{\xi}, u_{\xi_{j} \xi_{j}}^{\xi}$ and $u_{x}^{\xi}=-\sum_{j} u_{\xi_{j}}^{\xi}$ and $u_{x x}^{\xi}=\sum_{j} u_{\xi_{j} \xi_{j}}^{\xi}$.

5. Set

$$
p_{i j}(x)=\frac{\left|u_{\xi_{i}}^{\xi}(x) \cdot u_{\xi_{j}}^{\xi}(x)\right|}{\left\|u_{\xi_{i}}^{\xi}\right\|\left\|u_{\xi_{j}}^{\xi}\right\|}, i \neq j .
$$

To estimate $p_{i j}$ we can assume $\xi_{i}<\xi_{j}$. Observe that $u_{\xi_{i}}^{\xi}$ and $u_{\xi_{j}}^{\xi}$ are periodic maps which, for small $\epsilon>0$, concentrate at $\xi_{i}+\mathbb{Z}$ and $\xi_{j}+\mathbb{Z}$ respectively. Therefore we consider the two intervals $\left[\xi_{i}, \xi_{j}\right]$ and $\left[\xi_{j}, \xi_{i}+1\right]$. Set

$$
\bar{p}_{i j}(x)=\frac{\left|\bar{u}_{i}^{\prime}\left(\frac{x-\xi_{i}}{\epsilon}\right) \cdot \bar{u}_{j}^{\prime}\left(\frac{x-\xi_{j}}{\epsilon}\right)\right|}{\left\|\epsilon u_{\xi_{i}}^{\xi}\right\|\left\|\epsilon u_{\xi_{j}}^{\xi}\right\|},
$$

then we have

$$
p_{i j}(x) \leq \bar{p}_{i j}(x)+C \frac{\left|\varkappa_{i}\right|+\left|\varkappa_{j}\right|}{\left\|\epsilon u_{\xi_{i}}^{\xi}\right\|\left\|\epsilon u_{\xi_{j}}^{\xi}\right\|} .
$$

From (1.8) we have

$$
\begin{aligned}
& \bar{p}_{i j}(x) \leq \frac{C}{\epsilon} e^{-\frac{\mu_{i+1}^{+}}{\epsilon}\left(x-\xi_{i}\right)} e^{-\frac{\mu_{j}^{-}}{\epsilon}\left(\xi_{j}-x\right)} \leq \frac{C}{\epsilon}\left(e^{-\frac{\mu_{j}^{-}}{\epsilon}\left(\xi_{j}-\xi_{i}\right)}+e^{-\frac{\mu_{i+1}^{+}}{\epsilon}\left(\xi_{j}-\xi_{i}\right)}\right) \\
& \leq \frac{C}{\epsilon}\left(\mathscr{E}_{j}^{-}+\mathscr{E}_{i+1}^{+}\right), \quad x \in\left[\xi_{i}, \xi_{j}\right]
\end{aligned}
$$

where we have used the fact that a linear function assumes the maximum at the boundary of the interval of definition and also used $\xi_{j} \geq \xi_{i+1}$. In the same way one finds that in the interval $\left[\xi_{j}, \xi_{i}+1\right]$ it results

$$
\bar{p}_{i j} \leq \frac{C}{\epsilon}\left(\mathscr{E}_{i}^{-}+\mathscr{E}_{j+1}^{+}\right)
$$

This, (2.74), (2.73), (2.33) 1 and the bound for $\varkappa_{j}$ conclude the proof of (2.35).

6. From $u_{x}^{\xi}=-\sum_{j} u_{\xi_{j}}^{\xi}$ and (2.35) we have

$$
u_{x}^{\xi} \cdot u_{x}^{\xi}=\sum_{j} u_{\xi_{j}}^{\xi} \cdot u_{\xi_{j}}^{\xi}+\mathrm{O}\left(\frac{1}{\epsilon} \max _{j, \pm} \mathscr{E}_{j}^{ \pm}\right) .
$$


From this (2.31) and (2.71) it follows

$$
\begin{aligned}
& \epsilon^{2}\left\|u_{x}^{\xi}\right\|^{2}=\epsilon^{2} \sum_{j}\left\|u_{\xi_{j}}^{\xi}\right\|^{2}+\mathrm{O}\left(\epsilon \max _{j, \pm} \mathscr{E}_{j}^{ \pm}\right) \\
& =\sum_{j}\left\|\bar{u}_{j}^{\prime}\left(\frac{-\xi_{j}}{\epsilon}\right)\right\|^{2}+\mathrm{O}\left(\epsilon \max _{j, \pm} \mathscr{E}_{j}^{ \pm}\right)=\epsilon \sum_{j} \bar{q}_{j}^{2}+\mathrm{O}\left(\epsilon \max _{j, \pm} \mathscr{E}_{j}^{ \pm}\right) .
\end{aligned}
$$

We also observe that from (2.29) and Lemma 2.6 it follows

$$
\begin{aligned}
& x \in\left[\hat{\xi}_{j-1}, \hat{\xi}_{j}\right] \Rightarrow \\
& W\left(u^{\xi}\right)=W\left(\bar{u}_{j}\left(\frac{x-\xi_{j}}{\epsilon}\right)\right)+\mathrm{O}\left(e^{-\frac{\mu_{j}^{+}}{\epsilon}\left(x-\xi_{j-1}\right)}+e^{\frac{\mu_{j+1}^{-}}{\epsilon}\left(x-\xi_{j+1}\right)}+e^{-\frac{\alpha \rho}{\epsilon}} \max _{h, \pm} \mathscr{E}_{h}^{ \pm}\right),
\end{aligned}
$$

which yields

$$
\begin{aligned}
& \int_{\hat{\xi}_{j-1}}^{\hat{\xi}_{j}} W\left(u^{\xi}\right) d x=\epsilon \int_{-\frac{\left(\xi_{j}-\xi_{j-1}\right)}{2 \epsilon}}^{\frac{\left(\xi_{j+1}-\xi_{j}\right)}{2 \epsilon}} W\left(\bar{u}_{j}(s)\right) d s+\mathrm{O}\left(\epsilon \max _{h, \pm} \mathscr{E}_{h}^{ \pm \frac{1}{2}}\right) \\
& =\epsilon \int_{\mathbb{R}} W\left(\bar{u}_{j}(s)\right) d s+\mathrm{O}\left(\epsilon \max _{h, \pm} \mathscr{E}_{h}^{ \pm \frac{1}{2}}\right)=\frac{\epsilon}{2} \bar{q}_{j}^{2}+\mathrm{O}\left(\epsilon \max _{h, \pm} \mathscr{E}_{h}^{ \pm \frac{1}{2}}\right),
\end{aligned}
$$

where we have also used the fact that the heteroclinic solution $\bar{u}_{j}$ satisfies the equipartition of energy. This completes the proof of (2.34) and concludes the proof of the lemma.

Proof. (Lemma 2.9) We begin by deriving an $L^{\infty}$ bound for the projection $\gamma_{j}^{\xi}$ of $\epsilon u_{\xi_{j}}^{\xi}$ on $X^{\xi, \top}$.

Lemma 2.11. Let $\gamma_{j}^{\xi} \in X^{\xi, \perp}$ be defined by the condition that $\epsilon u_{\xi_{j}}^{\xi}+\gamma_{j}^{\xi} \in X^{\xi}$. Then, provided $\epsilon>0$ is sufficiently small, it results

$$
\begin{gathered}
\left\|\gamma_{j}^{\xi}\right\|_{W_{\epsilon}^{1,2}} \leq C \max _{h, \pm} \mathscr{E}_{h}^{ \pm}, \\
\left\|\gamma_{j}^{\xi}\right\|_{L^{\infty}} \leq \frac{C}{\epsilon^{\frac{1}{2}}} \max _{h, \pm} \mathscr{E}_{h}^{ \pm} .
\end{gathered}
$$

Proof. In this proof we simply write $\gamma$ instead of $\gamma_{j}^{\xi}$. The condition $\epsilon u_{\xi_{j}}^{\xi}+\gamma \in X^{\xi}$ can be expressed in the form

$$
\epsilon u_{\xi_{j}}^{\xi}+\gamma=\epsilon \sum_{h=1}^{N}\left\langle u_{\xi_{j}}^{\xi}, \varphi_{h}^{\xi}\right\rangle \varphi_{h}^{\xi},
$$

where as before $\varphi_{h}^{\xi}, h=1, \ldots, N$ is an orthonormal basis for $X^{\xi}$. Since $X^{\xi}$ is invariant under $L^{\xi}$ and $\gamma \in X^{\xi, \perp}$ we have

$$
L^{\xi}\left(\epsilon u_{\xi_{j}}^{\xi}+\gamma\right)=\epsilon \sum_{h=1}^{N}\left\langle u_{\xi_{j}}^{\xi}, \varphi_{h}^{\xi}\right\rangle L^{\xi} \varphi_{h}^{\xi} .
$$

Forming the inner product of this equation with $\gamma$ and using

$$
\begin{aligned}
& \left\langle L^{\xi} \varphi_{h}^{\xi}, \gamma\right\rangle=0, \\
& L^{\xi} u_{\xi_{j}}^{\xi}=-\mathscr{F}_{\xi_{j}}\left(u^{\xi}\right),
\end{aligned}
$$


on the basis of (2.5) in Proposition 2.1, we get

$$
\begin{aligned}
& \mu^{*}\|\gamma\|_{W_{\epsilon}^{1,2}}^{2} \leq\left\langle L^{\xi} \gamma, \gamma\right\rangle=\epsilon\left\langle\mathscr{F}_{\xi_{j}}\left(u^{\xi}\right), \gamma\right\rangle \\
& \leq \epsilon\left\|\mathscr{F}_{\xi_{j}}\left(u^{\xi}\right)\right\|\|\gamma\| \leq \epsilon\left\|\mathscr{F}_{\xi_{j}}\left(u^{\xi}\right)\right\|\|\gamma\|_{W_{\epsilon}^{1,2}}
\end{aligned}
$$

and therefore

$$
\begin{gathered}
\|\gamma\|_{W_{\epsilon}^{1,2}} \leq \frac{\epsilon}{\mu^{*}}\left\|\mathscr{F}_{\xi_{j}}\left(u^{\xi}\right)\right\|, \\
\|\gamma\|_{L^{\infty}} \leq \frac{\epsilon^{\frac{1}{2}}}{\mu^{*}}\left\|\mathscr{F}_{\xi_{j}}\left(u^{\xi}\right)\right\| .
\end{gathered}
$$

This and Lemma 2.7 concludes the proof.

We continue with the proof of Lemma 2.9. In the remaining part of the proof we drop the superscript $\xi$. Define

$$
v_{j}=\frac{u_{\xi_{j}}^{\xi}}{\left\|u_{\xi_{j}}^{\xi}\right\|}, \quad v_{i j}=\left|\left\langle v_{i}, v_{j}\right\rangle\right|, i \neq j, \quad w_{j}=\frac{\gamma_{j}^{\xi}}{\left\|\epsilon u_{\xi_{j}}^{\xi}\right\|}, \quad j=1, \ldots, N,
$$

where $\gamma_{j}^{\xi}$ is defined in Lemma 2.11, Note that Lemma 2.11, (2.33) 1 and (2.35) imply

$$
\begin{gathered}
\left\|w_{j}\right\| \leq \frac{C}{\epsilon^{\frac{1}{2}}} \max _{h, \pm} \mathscr{E}_{h}^{ \pm}, \\
v_{i j} \leq \frac{C}{\epsilon} \max _{h, \pm} \mathscr{E}_{h}^{ \pm} .
\end{gathered}
$$

The proof is based on Gram-Schmidt orthonormalization process applied to the vectors $v_{j}+w_{j}$ that on the basis of Lemma 2.11 belong to $X^{\xi}$. We have

$$
\begin{aligned}
\varphi_{1} & =\frac{v_{1}+w_{1}}{\left\|v_{1}+w_{1}\right\|}=v_{1}+\hat{w}_{1}, \\
\hat{w}_{1} & =v_{1} \frac{1-\left\|v_{1}+w_{1}\right\|}{\left\|v_{1}+w_{1}\right\|}+\frac{w_{1}}{\left\|v_{1}+w_{1}\right\|},
\end{aligned}
$$

and therefore

$$
\left\|\hat{w}_{1}\right\| \leq \frac{2\left\|w_{1}\right\|}{\left\|v_{1}+w_{1}\right\|} \leq 4\left\|w_{1}\right\|
$$

where we have used (2.76) and $\left\|v_{1}\right\|=1$ that imply $\left\|v_{1}+w_{1}\right\| \geq \frac{1}{2}$. Let $\psi_{n}, \tilde{w}_{n}$ and $\hat{w}_{n}$ be defined by

$$
\begin{aligned}
& \psi_{n}=v_{n}+w_{n}-\sum_{k=1}^{n-1}\left\langle v_{n}+w_{n}, \varphi_{k}\right\rangle \varphi_{k}=v_{n}+\tilde{w}_{n}, \\
& \varphi_{n}=\frac{v_{n}+\tilde{w}_{n}}{\left\|v_{n}+\tilde{w}_{n}\right\|}=v_{n}+\hat{w}_{n} .
\end{aligned}
$$


Then there is $C_{n}>0$ such that, provided $\epsilon>0$ is sufficiently small

$$
\begin{aligned}
& \left\|\hat{w}_{n}\right\| \leq 4\left\|\tilde{w}_{n}\right\|, \\
& \left\|\tilde{w}_{n}\right\| \leq C_{n}\left(\sum_{k=1}^{n}\left\|w_{k}\right\|+\sum_{k=2}^{n} \sum_{h=1}^{k-1} v_{h k}\right) .
\end{aligned}
$$

It suffices to prove the second inequality. Indeed, if (2.77) 2 holds and $\epsilon>0$ is small, (2.77) 1 follows from (2.76) and the same argument that shows $\left\|\hat{w}_{1}\right\| \leq 4\left\|w_{1}\right\|$. We prove the second inequality by induction. For $n=2$, observing also that $\tilde{w}_{1}=w_{1}$, we have

$$
\psi_{2}=v_{2}+w_{2}-\left\langle v_{2}+w_{2}, v_{1}+\hat{w}_{1}\right\rangle \varphi_{1}
$$

It follows, using also that $\left\langle w_{2}, \varphi_{1}\right\rangle=0$,

$$
\begin{aligned}
& \tilde{w}_{2}=w_{2}-\left\langle v_{2}, v_{1}\right\rangle \varphi_{1}-\left\langle v_{2}, \hat{w}_{1}\right\rangle \varphi_{1} \\
& \left\|\tilde{w}_{2}\right\| \leq\left\|w_{2}\right\|+\left\|\hat{w}_{1}\right\|+v_{12} \leq\left\|w_{2}\right\|+C\left\|w_{1}\right\|+v_{12}
\end{aligned}
$$

which shows that $(2.77)_{2}$ holds for $n=2$ with a suitable choice of $C_{2}$. Now we show that if (2.77) 2 holds for $2, \ldots, n$ then it also holds for $n+1$. From

$$
\begin{aligned}
& \psi_{n+1}=v_{n+1}+w_{n+1}-\sum_{k=1}^{n}\left\langle v_{n+1}+w_{n+1}, v_{k}+\hat{w}_{k}\right\rangle \varphi_{k} \\
& =v_{n+1}+w_{n+1}-\sum_{k=1}^{n}\left(\left\langle w_{n+1}, \varphi_{k}\right\rangle+\left\langle v_{n+1}, v_{k}\right\rangle+\left\langle v_{n+1}, \hat{w}_{k}\right\rangle\right) \varphi_{k}
\end{aligned}
$$

we obtain, recalling also that $\left\langle w_{n+1}, \varphi_{k}\right\rangle=0, k=1, \ldots, n$,

$$
\begin{aligned}
& \left\|\tilde{w}_{n+1}\right\| \leq\left\|w_{n+1}\right\|+\sum_{k=1}^{n} v_{k, n+1}+\sum_{k=1}^{n}\left\|\hat{w}_{k}\right\| \\
& \leq\left\|w_{n+1}\right\|+\sum_{k=1}^{n} v_{k, n+1}+4 \sum_{k=1}^{n}\left\|\tilde{w}_{k}\right\| \\
& \leq\left\|w_{n+1}\right\|+\sum_{k=1}^{n} v_{k, n+1}+4 \sum_{k=1}^{n} C_{k}\left(\sum_{j=1}^{k}\left\|w_{j}\right\|+\sum_{i=2}^{k} \sum_{h=1}^{i-1} v_{h i}\right),
\end{aligned}
$$

where, for the last inequality, we have used $(2.77)_{2}$ for $2, \ldots, n$. The inequality (2.78) establishes $(2.77)_{2}$ for $n+1$ for a suitable choice of $C_{n+1}$. With the identification $\eta_{j}^{\xi}=\hat{w}_{j}$, the definition of $v_{j}$ and $\hat{w}_{j}$ imply that $\varphi_{j}^{\xi}=\frac{u_{\xi_{j}}^{\xi}}{\left\|u_{\xi_{j}}^{\xi}\right\|}+\eta_{j}^{\xi}, j=1, \ldots, N$ is an orthonornal basis for the subspace $X^{\xi}$. The estimate for $\eta_{j}^{\xi}$ follows from (2.77) and (2.76). The proof is complete.

\section{Layers Dynamics}

We now focus on the dynamics of the parabolic equations

$$
u_{t}=\epsilon^{2} u_{x x}-W_{u}(u), \quad x \in(0,1), u \in W^{1,2},
$$


on the set of 1-periodic maps. As we have indicated in the introduction we expect that $c(\xi)$ determines the dynamics of (3.1) in a neighborhood of the manifold $\mathcal{M}=\left\{u^{\xi}: \xi \in \Xi_{\rho}\right\}$ and therefore layers dynamics since maps in a $W^{1,2}$ neighborhood of $\mathcal{M}$ have a layered structure. The scope of this section is to prove that this is indeed the case. We begin by defining a smooth change of coordinate in a neighborhood of $\mathcal{M}$. As before we denote $L^{2}$ and $W^{1,2}$ the corresponding subsets of 1-periodic maps. For $\eta>0, \rho>0$ small set

$$
\mathscr{U}_{\rho}^{\eta}=\left\{u \in W^{1,2}: \inf _{\zeta \in \Xi_{\rho}}\left\|u-u^{\zeta}\right\|_{W_{\epsilon}^{1,2}}<\eta\right\} .
$$

Lemma 3.1. Set $\eta=a \epsilon^{\frac{1}{2}}$. Then, if $a>0$ is sufficiently small, there is a constant $c>0$ such that, for each $u \in \mathscr{U}_{\rho}^{a \epsilon^{\frac{1}{2}}}$ there is a unique $\xi=\xi(u) \in \Xi_{\rho-c \epsilon}$ that satisfies

$$
\begin{aligned}
& \left\langle u-u^{\xi}, u_{\xi_{i}}^{\xi}\right\rangle=0, \quad i=1, \ldots, N \\
& \left\|u-u^{\xi}\right\| \leq \inf _{\zeta \in \Xi_{\rho}}\left\|u-u^{\zeta}\right\|_{W_{\epsilon}^{1,2}}
\end{aligned}
$$

Moreover

$$
\left\|u-u^{\xi}\right\|_{W_{\epsilon}^{1,2}} \leq \bar{C} \inf _{\zeta \in \Xi_{\rho}}\left\|u-u^{\zeta}\right\|_{W_{\epsilon}^{1,2}},
$$

for some constant $\bar{C}>0$, independent of $\epsilon>0$.

Proof. We need the following result that we prove in Section 3.2 ,

Lemma 3.2. There are positive constants $\omega_{0}, \omega_{1}, C_{ \pm}^{0}, C_{ \pm}^{1}$ and positive maps $m_{0}:\left(0, \omega_{0}\right] \rightarrow$ $\mathbb{R}, m_{1}:\left(0, \omega_{1}\right] \rightarrow \mathbb{R}$ such that, for $\xi, \zeta \in \Xi_{\rho}$, it results

$$
\begin{aligned}
& |\zeta-\xi| \geq \omega \epsilon \Rightarrow\left\|u^{\zeta}-u^{\xi}\right\|_{L^{\infty}} \geq m_{1}(\omega), \omega \in\left(0, \omega_{1}\right], \\
& |\zeta-\xi| \leq \omega_{1} \epsilon \Rightarrow \frac{C_{-}^{1}}{\epsilon}|\zeta-\xi| \leq\left\|u^{\zeta}-u^{\xi}\right\|_{L^{\infty}} \leq \frac{C_{+}^{1}}{\epsilon}|\zeta-\xi|,
\end{aligned}
$$

and

$$
\begin{aligned}
& |\zeta-\xi| \geq \omega \epsilon \Rightarrow\left\|u^{\zeta}-u^{\xi}\right\| \geq m_{0}(\omega) \epsilon^{\frac{1}{2}}, \omega \in\left(0, \omega_{0}\right], \\
& |\zeta-\xi| \leq \omega_{0} \epsilon \Rightarrow \frac{C_{-}^{0}}{\epsilon^{\frac{1}{2}}}|\zeta-\xi| \leq\left\|u^{\zeta}-u^{\xi}\right\| \leq \frac{C_{+}^{0}}{\epsilon^{\frac{1}{2}}}|\zeta-\xi| .
\end{aligned}
$$

Moreover

$$
|\zeta-\xi| \leq \omega_{0} \epsilon \Rightarrow\left\|u_{x}^{\zeta}-u_{x}^{\xi}\right\| \leq \frac{C_{+}^{0}}{\epsilon^{\frac{3}{2}}}|\zeta-\xi|
$$

1. From the definition of $\mathscr{U}_{\rho}^{a \epsilon^{\frac{1}{2}}}$ we can associate to each $u \in \mathscr{U}_{\rho}^{a \epsilon^{\frac{1}{2}}}$ a $\bar{\xi} \in \Xi_{\rho}$ such that $\left\|u-u^{\bar{\xi}}\right\|<a \epsilon^{\frac{1}{2}}$. Let $\omega \in\left(0, \omega_{0}\right]$ a number to be chosen later. For $a \in\left(0, \frac{1}{2} m_{0}\left(\frac{\omega}{2}\right)\right], m_{0}$ as in (3.6), the problem

$$
\left\|u-u^{\xi}\right\|=\min _{\zeta \in \bar{B}_{\omega \epsilon}(\bar{\xi})}\left\|u-u^{\zeta}\right\|<a \epsilon^{\frac{1}{2}},
$$

has a solution $\xi \in B_{\frac{\omega}{2}} \epsilon(\bar{\xi})$. 
Existence follows from the compactness of $\bar{B}_{\omega \epsilon}(\bar{\xi})$ and the continuity of the map $\zeta \rightarrow$ $\left\|u-u^{\zeta}\right\|$. Lemma 3.2, $a \in\left(0, \frac{1}{2} m_{0}\left(\frac{\omega}{2}\right)\right]$, and $|\zeta-\bar{\xi}| \geq \frac{\omega}{2} \epsilon$ imply

$$
\left\|u-u^{\zeta}\right\| \geq\left\|u^{\bar{\xi}}-u^{\zeta}\right\|-\left\|u-u^{\bar{\xi}}\right\| \geq\left(m_{0}\left(\frac{\omega}{2}\right)-a\right) \epsilon^{\frac{1}{2}} \geq a \epsilon^{\frac{1}{2}} .
$$

This implies $\left.\xi \in B_{\frac{\omega}{2} \epsilon} \epsilon \bar{\xi}\right)$ and concludes the proof of 1 .

Note that while $\bar{\xi} \in \Xi_{\rho}$ it may occur that $B_{\frac{\omega}{2}} \epsilon(\bar{\xi}) \backslash \Xi_{\rho} \neq \emptyset$. Therefore it may happen that $\xi \notin \Xi_{\rho}$ but, since $|\xi-\bar{\xi}|<\frac{\omega}{2} \epsilon$, there is a constant $c>0$ such that $\xi \in \Xi_{\rho-c \epsilon}$.

2. The minimizer $\xi$ determined in 1 . satisfies (3.3).

The fact that $\xi$ satisfies $(3.3)_{2}$ follows from (3.8) and (3.9) that imply

$$
\left\|u-u^{\xi}\right\|=\min _{\zeta \in \Xi_{\rho}}\left\|u-u^{\zeta}\right\| .
$$

On the other hand, since $\xi$ belong to the interior of $B_{\omega \epsilon}(\bar{\xi}),\left({ }_{3.3}\right)_{1}$ follows from the minimality of $\xi$ and a standard argument.

3. The solution $\xi$ of (3.3) is unique, independent from the auxiliary point $\bar{\xi}$ and is Lipschitz continuous on $u \in L^{2}$.

Given $u, v \in \mathscr{U}_{\rho}^{a \epsilon^{\frac{1}{2}}}$ let $\xi$ and $\eta$ be the corresponding solutions of (3.3) claimed in 2. We have

$$
\begin{aligned}
& 0=\left(\left\langle u-u^{\xi}, u_{\xi_{j}}^{\xi}\right\rangle\right)-\left(\left\langle v-u^{\eta}, u_{\xi_{j}}^{\eta}\right\rangle\right) \\
& =\left(\left\langle u^{\eta}-u^{\xi}, u_{\xi_{j}}^{\xi}\right\rangle\right)+\left(\left\langle u-v, u_{\xi_{j}}^{\xi}\right\rangle\right)+\left(\left\langle v-u^{\eta}, u_{\xi_{j}}^{\xi}-u_{\xi_{j}}^{\eta}\right\rangle\right),
\end{aligned}
$$

where $\left(w_{i}\right) \subset \mathbb{R}^{N}$ denotes the vector with components $w_{1}, \ldots, w_{N}$.

Assume that $|\xi-\eta| \leq \omega \epsilon$. Then from (3.52), (3.53), (3.55) (cfr. the proof of Lemma 3.2 and (2.31) we obtain

$$
\left|\left\langle u^{\zeta}-u^{\xi}, u_{\xi_{j}}^{\xi}\right\rangle-\frac{\bar{q}_{j}^{2}}{\epsilon}\left(\eta_{j}-\xi_{j}\right)\right| \leq \frac{C}{\epsilon^{2}}|\zeta-\xi|^{2}+\mathrm{O}\left(e^{-\frac{\alpha \rho}{\epsilon}}\right)|\zeta-\xi|,
$$

and therefore, if we take $\omega=\frac{\bar{q}_{m}^{2}}{2 C}, \bar{q}_{m}=\min _{h} \bar{q}_{h}$, we have

$$
\begin{aligned}
& \left|\left(\left\langle u^{\eta}-u^{\xi}, u_{\xi_{j}}^{\xi}\right\rangle\right)\right| \geq \frac{\bar{q}_{m}^{2}}{\epsilon}|\eta-\xi|-\frac{C}{\epsilon^{2}}|\eta-\xi|^{2} \\
& \geq \frac{\bar{q}_{m}^{2}-C \omega}{\epsilon}|\eta-\xi| \geq \frac{\bar{q}_{m}^{2}}{2 \epsilon}|\eta-\xi|
\end{aligned}
$$

A similar computation yields

$$
\left\|u_{\xi_{j}}^{\xi}-u_{\xi_{j}}^{\eta}\right\| \leq \frac{C}{\epsilon^{\frac{3}{2}}}|\eta-\xi|,
$$

and we obtain

$$
\left|\left(\left\langle v-u^{\eta}, u_{\xi_{j}}^{\xi}-u_{\xi_{j}}^{\eta}\right\rangle\right)\right| \leq \frac{C a}{\epsilon}|\eta-\xi|
$$

We also have

$$
\left|\left(\left\langle u-v, u_{\xi_{j}}^{\xi}\right\rangle\right)\right| \leq \frac{C}{\epsilon^{\frac{1}{2}}}\|u-v\|
$$


From these estimates and (3.10) it follows

$$
0 \geq \frac{\frac{q_{m}^{2}}{2}-C a}{\epsilon}|\eta-\xi|-\frac{C}{\epsilon^{\frac{1}{2}}}\|u-v\| \geq \frac{q_{m}^{2}}{4 \epsilon}|\eta-\xi|-\frac{C}{\epsilon^{\frac{1}{2}}}\|u-v\|,
$$

where $a \in\left(0, \frac{q_{m}^{2}}{4 C}\right]$.

From (3.9) if a minimizer of (3.3) 2 exists it belongs to $B_{\frac{\omega}{2} \epsilon}(\bar{\xi})$. Therefore if $\xi$ and $\eta$ are solutions of (3.3) corresponding to the same $u \in \mathscr{U}_{\rho}^{a \epsilon^{\frac{1}{2}}}$ we have $|\eta-\xi|<\omega \epsilon$ from (3.9) and we can apply (3.11) with $u=v$ that yields $\eta=\xi$. This establishes uniqueness. The same argument implies $\xi=\eta$ if $\xi$ and $\eta$ are minimizers of (3.8) corresponding to two different auxiliary points $\bar{\xi}$ and $\bar{\eta}$. Clearly (3.11) also yields the Lipschitz continuity of the solution of (3.3).

To complete the proof set $\gamma=\inf _{\zeta \in \Xi_{\rho}}\left\|u-u^{\zeta}\right\|_{W_{\epsilon}^{1,2}}$. Since we have $\gamma<a \epsilon^{\frac{1}{2}}$ we can fix a $\bar{\xi} \in \Xi_{\rho}$ that satisfies

$$
\left\|u-u^{\bar{\xi}}\right\|_{W_{\epsilon}^{1,2}} \leq k \gamma<a \epsilon^{\frac{1}{2}},
$$

for some $k \in(1,2]$. This and $\left\|u-u^{\xi}\right\| \leq \gamma$ that follows from $(3.3)_{2}$ imply $\left\|u^{\bar{\xi}}-u^{\xi}\right\| \leq$ $(1+k) \gamma$. From the first part of the proof we also have $|\xi-\bar{\xi}|<\omega_{0} \epsilon$ and we can apply $(\sqrt{3.6})_{2}$ in Lemma 3.2 that yields $\frac{C_{-}^{0}}{\epsilon^{\frac{1}{2}}}|\bar{\xi}-\xi| \leq(1+k) \gamma$ and in turn

$$
\begin{gathered}
\epsilon\left\|u_{x}^{\bar{\xi}}-u_{x}^{\xi}\right\| \leq \frac{C_{+}^{0}}{\epsilon^{\frac{1}{2}}}|\bar{\xi}-\xi| \leq \frac{C_{+}^{0}}{C_{-}^{0}}(1+k) \gamma, \\
\Rightarrow \quad\left\|u^{\bar{\xi}}-u^{\xi}\right\|_{W_{\epsilon}^{1,2}} \leq\left(1+\frac{C_{+}^{0}}{C_{-}^{0}}\right)(1+k) \gamma,
\end{gathered}
$$

and, using also $k \leq 2$, we finally obtain

$$
\left\|u-u^{\xi}\right\|_{W_{\epsilon}^{1,2}} \leq\left\|u-u^{\bar{\xi}}\right\|_{W_{\epsilon}^{1,2}}+\left\|u^{\bar{\xi}}-u^{\xi}\right\|_{W_{\epsilon}^{1,2}} \leq\left(2+3\left(1+\frac{C_{+}^{0}}{C_{-}^{0}}\right)\right) \gamma,
$$

that prove the last statement of the Lemma. The proof is complete.

Set

$$
\begin{aligned}
& \mathscr{W}_{\rho}^{a \epsilon^{\frac{1}{2}}}=\left\{(\xi, w): \xi \in \Xi_{\rho}, w \in W^{1,2},\|w\|_{W_{\epsilon}^{1,2}}<a \epsilon^{\frac{1}{2}},\left(\left\langle w, u_{\xi_{j}}^{\xi}\right\rangle\right)=0\right\}, \\
& \mathscr{U}\left(\mathscr{W}_{\rho}^{a \epsilon^{\frac{1}{2}}}\right)=\left\{u=u^{\xi}+w:(\xi, w) \in \mathscr{W}_{\rho}^{a \epsilon^{\frac{1}{2}}}\right\} .
\end{aligned}
$$

Lemma 3.1 implies that $\mathscr{U}\left(\mathscr{W}_{\rho}^{a \epsilon^{\frac{1}{2}}}\right) \subset \mathscr{U}_{\rho}^{a \epsilon^{\frac{1}{2}}}$ and $\mathscr{U}_{\rho}^{a \epsilon^{\frac{1}{2}}} \subset \mathscr{U}\left(\mathscr{W}_{\rho-c \epsilon}^{\bar{C} a \epsilon^{\frac{1}{2}}}\right)$ and that the map

$$
\mathscr{W}_{\rho}^{a \epsilon^{\frac{1}{2}}} \ni(\xi, w) \rightarrow u=u^{\xi}+w \in \mathscr{U}\left(\mathscr{W}_{\rho}^{a \epsilon^{\frac{1}{2}}}\right)
$$

is a surjection. Note also that $\mathscr{U}\left(\mathscr{W}_{\rho}^{a \epsilon^{\frac{1}{2}}}\right)$ is open in $W_{\epsilon}^{1,2}$. This follows from the fact that $\left(\xi_{0}, w_{0}\right) \in \mathscr{W}_{\rho}^{a \epsilon^{\frac{1}{2}}}$ implies $\xi_{0} \in \Xi_{\rho+\delta_{0}}$ and $\|w\|_{W_{\epsilon}^{1,2}}<a \epsilon^{\frac{1}{2}}-\delta_{0}$ for some $\delta_{0}>0$ that depends on $\left(\xi_{0}, w_{0}\right)$. In conclusion $\mathscr{U}\left(\mathscr{W}_{\rho}^{a \epsilon^{\frac{1}{2}}}\right)$ is a tubular neighborhood of the basic manifold $\mathcal{M}$ with a well defined system of coordinate and for each $u \in \mathscr{U}\left(\mathscr{W}_{\rho}^{a \epsilon^{\frac{1}{2}}}\right)$ there is a unique pair $\xi \in \Xi_{\rho}$ and $w \in W^{1,2}$ such that

$$
\begin{aligned}
& u=u^{\xi}+w, \\
& \left\langle w, u_{\xi_{j}}^{\xi}\right\rangle=0, \quad j=1, \ldots, N .
\end{aligned}
$$




\subsection{The proof of Theorems 1.2 and 1.3}

The proof of Theorem 1.2 is based on a geometric characterization of the energy functional $W^{1,2} \ni u \rightarrow J_{\epsilon}(u)=\int_{0}^{1}\left(\frac{\epsilon^{2}}{2}\left|u_{x}\right|^{2}+W(u)\right) d x$ for $u$ near $\mathcal{M}$. By mean of Theorem 2.1 in [11] we show that a map $u$ laying in a neighborhood of $\mathcal{M}$ of size $\eta^{*}=\mathrm{O}\left(\epsilon^{\frac{3}{2}}\right)$, if has energy $J_{\epsilon}(u)$ slightly larger than the energy of the maps $u^{\xi} \in \mathcal{M},\left(J_{\epsilon}(u)-J_{\epsilon}\left(u^{\xi}\right)=\mathrm{O}\left(e^{-\frac{\alpha \rho}{\epsilon}}\right)\right)$, actually lays in a much smaller neighborhood of size $\eta_{*}=\mathrm{O}\left(e^{-\frac{\alpha \rho}{\epsilon}}\right)$.

From Lemma 3.1 for $u \in \mathscr{U}\left(\mathscr{W}_{\rho}^{a \epsilon^{\frac{1}{2}}}\right)$ the change of variable $u \leftrightarrow(\xi, w)$ is well defined and the decomposition $u=u^{\xi}+w$ entails a decomposition of $J_{\epsilon}\left(u^{\xi}+w\right)-J_{\epsilon}\left(u^{\xi}\right)$ :

$$
J_{\epsilon}\left(u^{\xi}+w\right)-J_{\epsilon}\left(u^{\xi}\right)=\mathscr{L}^{\xi}(w)+\mathscr{Q}^{\xi}(w)+\mathscr{N}^{\xi}(w),
$$

where

$$
\begin{aligned}
& \mathscr{L}^{\xi}(w)=\int_{0}^{1}\left(\epsilon^{2} u_{x}^{\xi} \cdot w_{x}+W_{u}\left(u^{\xi}\right) w\right) d x, \\
& \mathscr{Q}^{\xi}(w)=\frac{1}{2} \int_{0}^{1}\left(\epsilon^{2}\left|w_{x}\right|^{2}+W_{u u}\left(u^{\xi}\right) w \cdot w\right) d x, \\
& \mathscr{N}^{\xi}(w)=\int_{0}^{1}\left(W\left(u^{\xi}+w\right)-W\left(u^{\xi}\right)-W_{u}\left(u^{\xi}\right)-\frac{1}{2} W_{u u}\left(u^{\xi}\right) w \cdot w\right) d x .
\end{aligned}
$$

Next we derive estimates that describe the geometry of the functional $u \rightarrow J_{\epsilon}(u)$ and allow for the application of Theorem 2.1 in [11].

We have

$$
\left|\mathscr{L}^{\xi}(w)\right| \leq \delta_{L}\|w\|_{W_{\epsilon}^{1,2}}, \quad \delta_{L}=\mathrm{O}\left(e^{-\frac{\alpha \rho}{\epsilon}}\right) .
$$

Indeed integrating by parts and using (2.36) yields

$$
\begin{aligned}
& \left|\mathscr{L}^{\xi}(w)\right|=\left|\int_{0}^{1} \mathscr{F}\left(u^{\xi}\right) \cdot w d x\right| \\
& \leq e^{-\frac{\alpha \rho}{\epsilon}} \max _{h, \pm} \mathscr{E}_{h}^{ \pm \frac{1}{2}}\|w\| \leq e^{-\frac{\alpha \rho}{\epsilon}}\|w\|_{W_{\epsilon}^{1,2}}
\end{aligned}
$$

Since $w \perp \operatorname{span}\left(\frac{u_{\xi_{1}}^{\xi}}{\left\|u_{\xi_{1}}^{\xi}\right\|}, \ldots, \frac{u_{\xi_{N}}^{\xi}}{\left\|u_{\xi_{N}}^{\xi}\right\|}\right)$ Lemma 3.3 below implies

$$
\mathscr{Q}^{\xi}(w)=\frac{1}{2}\left\langle L^{\xi} w, w\right\rangle \geq \frac{1}{2} \mu^{*}\|w\|_{W_{\epsilon}^{1,2}}^{2},
$$

To estimate $\mathscr{N}^{\xi}(w)$ we observe that $u \in \mathscr{U}\left(\mathscr{W}_{\rho}^{a \epsilon^{\frac{1}{2}}}\right)$ implies $\|w\|_{W_{\epsilon}^{1,2}} \leq a \epsilon^{\frac{1}{2}}$ and, arguing as in (2.13), we have

$$
\|w\|_{L^{\infty}} \leq C \epsilon^{-\frac{1}{2}}\|w\|_{W_{\epsilon}^{1,2}} .
$$

It follows $\|w\|_{L^{\infty}} \leq C$ and therefore $\left|W_{u u u}\left(u^{\xi}+w\right)\right| \leq C$ and we conclude

$$
\left|\mathscr{N}^{\xi}(w)\right| \leq C|w|^{3} \leq \frac{C}{\epsilon^{\frac{3}{2}}}\|w\|_{W_{\epsilon}^{1,2}}^{3} .
$$


From (2.34) we also have

$$
J_{\epsilon}\left(u^{\xi}\right)-J_{\epsilon}\left(u^{\bar{\xi}}\right) \leq \delta_{0}, \quad \xi, \bar{\xi} \in \Xi_{\rho}
$$

with

$$
\delta_{0} \leq C e^{-\frac{\alpha \rho}{\epsilon}}
$$

We restrict to the class of maps that satisfy

$$
\delta_{1} \geq J_{\epsilon}(u)-\sup _{\xi \in \Xi_{\rho}} J_{\epsilon}\left(u^{\xi}\right), \quad \delta_{1}=\mathrm{O}\left(e^{-\frac{\alpha \rho}{\epsilon}}\right) .
$$

Since the energy is nonincreasing along the flow generated by the parabolic equation (3.1), if this condition is satisfies at a certain time $\bar{t}$ then is satisfied for $t \geq \bar{t}$.

The estimates (3.15), (3.16), (3.18),(3.19) and the assumption (3.20) allow us to compute the numbers $\eta^{*}$ and $\eta_{*}$ defined in Theorem 2.1 in [11]. In the case at hand it results

$$
\eta^{*}=\frac{\mu^{*}}{C \bar{C}} \epsilon^{\frac{3}{2}}=C \epsilon^{\frac{3}{2}}, \quad \eta_{*}=\frac{\delta_{L}}{\mu^{*}}+\sqrt{\frac{\delta_{L}^{2}}{\mu^{*}}+2 \frac{\delta_{0}+\delta_{1}}{\mu^{*}}}=\mathrm{O}\left(e^{-\frac{\alpha \rho}{\epsilon}}\right),
$$

$(\bar{C}$ is the constant in (3.4) $)$. Therefore, on the basis of Theorem 2.1 in [11], we can state that, for $\epsilon>0$ sufficiently small,

$$
u=u^{\xi}+w \in \mathscr{U}\left(\mathscr{W}_{\rho}^{C \epsilon^{\frac{3}{2}}}\right), \delta_{1}=\mathrm{O}\left(e^{-\frac{\alpha \rho}{\epsilon}}\right) \Rightarrow\|w\|_{W_{\epsilon}^{1,2}}<C e^{-\frac{\alpha \rho}{\epsilon}} .
$$

Since (3.1) is the $L^{2}$-gradient system associated to the functional $J_{\epsilon}(u)$, if the initial datum $u_{0}=u^{\xi_{0}}+w_{0} \in \mathscr{U}\left(\mathscr{W}_{\rho}^{C \epsilon^{\frac{3}{2}}}\right)$ satisfies (3.20) , the same is true for the the solution $u\left(t, u_{0}\right)=u^{\xi(t)}+w(t)$ of (3.1) through $u_{0}$. It follows that $u\left(t, u_{0}\right)$ creeps along $\mathcal{M}$ and $w(t)$ satisfies (3.21) for $t \in[0, T)$ where either $T=+\infty$ or $\xi(T) \in \partial \Xi_{\rho}$ and a standard computation, see Theorem 2.2 in [11], yields

$$
T \geq e^{\frac{\alpha \rho}{\epsilon}}\left(d_{L^{2}}\left(u_{0}, \partial \mathcal{M}\right)-C \epsilon^{\frac{1}{2}}\right)^{2} \geq e^{\frac{\alpha \rho}{\epsilon}} d^{2}, \quad d=\inf _{\zeta \in \partial \Xi_{\rho}}\left\|u^{\xi_{0}}-u^{\zeta}\right\| .
$$

This concludes the proof of Theorem 1.2. In Fig. 4 we explain the meaning of the numbers $\eta^{*}$ and $\eta_{*}$ and sketch the qualitative structure of a cross section of the graph of $u \rightarrow J_{\epsilon}(u)$ for $u$ near $\mathcal{M}$.

We now continue with the proof of Theorem 1.3. On the basis of Theorem 1.2 we can assume that $u_{0}=u^{\xi_{0}}+w_{0}$ is chosen so that $u\left(t, u_{0}\right)$ remains in $\mathscr{U}\left(\mathscr{W}_{\rho}^{C \epsilon^{\frac{3}{2}}}\right)$ and (3.21) holds for an exponentially long time. In particular we have that the change of variables $u \rightarrow(\xi, w)$ is well defined along the motion and (3.1) is equivalent to

$$
\begin{aligned}
& w_{t}+\sum_{h=1}^{N} u_{\xi_{h}}^{\xi} \dot{\xi}_{h}=\mathscr{F}\left(u^{\xi}+w\right) \\
& =\mathscr{F}\left(u^{\xi}\right)+\mathscr{F}\left(u^{\xi}+w\right)-\mathscr{F}\left(u^{\xi}\right) \\
& =\mathscr{F}\left(u^{\xi}\right)-L^{\xi} w-N^{\xi}(w),
\end{aligned}
$$




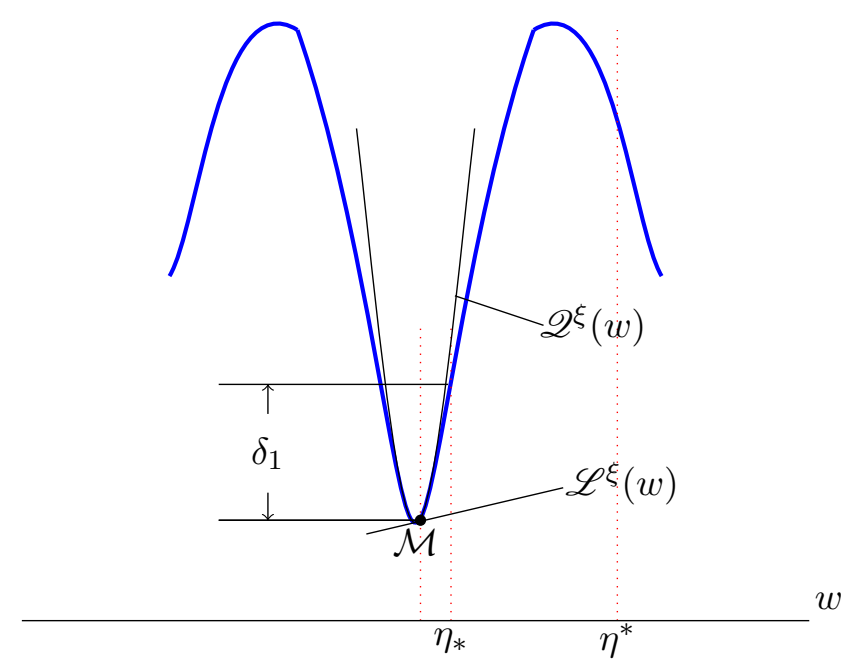

Figure 4: The structure of $J_{\epsilon}(u)$ near $\mathcal{M}$.

where $N^{\xi}(w)=W_{u}\left(u^{\xi}+w\right)-W_{u}\left(u^{\xi}\right)-W_{u u}\left(u^{\xi}\right) w$. Now, using (3.22), we derive an equation for the evolution of $\left\langle w, L^{\xi} w\right\rangle=\int_{0}^{1}\left(\epsilon^{2}\left|w_{x}\right|^{2}+W_{u u}\left(u^{\xi}\right) w \cdot w\right) d x$. We have

$$
\begin{aligned}
& \frac{1}{2} \frac{d}{d t}\left\langle w, L^{\xi} w\right\rangle=\left\langle L^{\xi} w, w_{t}\right\rangle+\frac{1}{2} \int_{0}^{1} W_{\text {uиu }}\left(u^{\xi}\right) \sum_{h=1}^{N} u_{\xi_{h}}^{\xi} \dot{\xi}_{h} w \cdot w d x \\
& =-\left\|L^{\xi} w\right\|^{2}-\left\langle L^{\xi} w, N^{\xi}(w)\right\rangle+\left\langle L^{\xi} w, \mathscr{F}\left(u^{\xi}\right)-\sum_{h=1}^{N} u_{\xi_{h}}^{\xi} \dot{\xi}_{h}\right\rangle \\
& +\frac{1}{2} \int_{0}^{1} W_{\text {uиu }}\left(u^{\xi}\right) \sum_{h=1}^{N} u_{\xi_{h}}^{\xi} \dot{\xi}_{h} w \cdot w d x .
\end{aligned}
$$

Differentiating $(\underline{3.12})_{2}$ we get the identity

$$
\left\langle w_{t}, u_{\xi_{j}}^{\xi}\right\rangle+\sum_{h=1}^{N}\left\langle w, u_{\xi_{j} \xi_{h}}^{\xi}\right\rangle \dot{\xi}_{h}=0, \quad j=1, \ldots, N
$$

Inserting the expression of $w_{t}$ given by (3.22) into (3.24) yields

$$
\sum_{h=1}^{N}\left(\left\langle u_{\xi_{h}}^{\xi}, u_{\xi_{j}}^{\xi}\right\rangle-\left\langle w, u_{\xi_{j} \xi_{h}}^{\xi}\right\rangle\right) \dot{\xi}_{h}=\left\langle\mathscr{F}\left(u^{\xi}\right)-L^{\xi} w-N^{\xi}(w), u_{\xi_{j}}^{\xi}\right\rangle, \quad j=1, \ldots, N,
$$

We rewrite (3.25) in the form

$$
\dot{\xi}_{j}=\frac{1}{\left\|u_{\xi_{j}}^{\xi}\right\|^{2}}\left\langle\mathscr{F}\left(u^{\xi}\right)-L^{\xi} w-N^{\xi}(w), u_{\xi_{j}}^{\xi}\right\rangle+\frac{\left\langle w, u_{\xi_{j} \xi_{j}}^{\xi}\right\rangle}{\left\|u_{\xi_{j}}^{\xi}\right\|^{2}} \dot{\xi}_{j}-\sum_{h \neq j} \frac{\left\langle u_{\xi_{j}}^{\xi}, u_{\xi_{h}}^{\xi}\right\rangle}{\left\|u_{\xi_{j}}^{\xi}\right\|^{2}} \dot{\xi}_{h},
$$

where we have used (2.31) that implies $u_{\xi_{j} \xi_{h}}^{\xi}=0$ for $h \neq j$. From (3.21) and (2.33) it follows that, for small $\epsilon>0$, this equation can be solved for $\dot{\xi}$. Note that by (2.47) 
$\left\langle\mathscr{F}\left(u^{\xi}\right), \frac{u_{\xi_{j}}^{\xi}}{\left\|u_{\xi_{j}}^{\xi}\right\|}\right\rangle=\bar{c}_{j}(\xi)$ and that $L^{\xi} u_{\xi_{j}}^{\xi}=-\mathscr{F}_{\xi_{j}}\left(u^{\xi}\right)$. From these observations and

$$
\left|N^{\xi}(w)\right|=\left|\int_{0}^{1} \int_{0}^{1} s W_{\text {uuu }}\left(u^{\xi}+\tau s w\right) w \cdot w d \tau d s\right| \leq C|w|^{2} \leq \frac{C}{\epsilon^{\frac{1}{2}}}\|w\|_{W_{\epsilon}^{1,2}|w|,}
$$

which implies

$$
\left|\left\langle N^{\xi}(w), \frac{u_{\xi_{j}}^{\xi}}{\left\|u_{\xi_{j}}^{\xi}\right\|}\right\rangle\right| \leq \frac{C}{\epsilon^{\frac{1}{2}}}\|w\|_{W_{\epsilon}^{1,2}}\|w\|,
$$

it follows

$$
\begin{aligned}
& \frac{1}{\left\|u_{\xi_{j}}^{\xi}\right\|^{2}}\left\langle\mathscr{F}\left(u^{\xi}\right)-L^{\xi} w-N^{\xi}(w), u_{\xi_{j}}^{\xi}\right\rangle \\
& =\frac{\bar{c}_{j}(\xi)}{\left\|u_{\xi_{j}}^{\xi}\right\|}+\mathrm{O}\left(\|w\|_{W_{\epsilon}^{1,2}}\left(\epsilon\left\|\mathscr{F}_{\xi_{j}}\left(u^{\xi}\right)\right\|+\|w\|_{W_{\epsilon}^{1,2}}\right)\right),
\end{aligned}
$$

where we have also used (2.33). Therefore by solving (3.26) we obtain

$$
\begin{aligned}
& \dot{\xi}_{j}=\frac{\bar{c}_{j}(\xi)}{\left\|u_{\xi_{j}}^{\xi}\right\|}+\mathrm{O}\left(\|w\|_{W_{\epsilon}^{1,2}}\left(\epsilon\left\|\mathscr{F}_{\xi_{j}}\left(u^{\xi}\right)\right\|+\|w\|_{W_{\epsilon}^{1,2}}\right)\right) \\
& +\mathrm{O}\left(e^{-\frac{\alpha \rho}{\epsilon}} \sum_{h}\left(\left|\bar{c}_{h}(\xi)\right|+\|w\|_{W_{\epsilon}^{1,2}}\left\|\mathscr{F}_{\xi_{h}}\left(u^{\xi}\right)\right\|\right)\right) .
\end{aligned}
$$

From (3.29), $\left|\bar{c}_{h}(\xi)\right| \leq\left\|\mathscr{F}\left(u^{\xi}\right)\right\|$, Lemma 2.7, and (3.21) it follows

$$
|\dot{\xi}| \leq C\left(\epsilon^{\frac{1}{2}}\left\|\mathscr{F}\left(u^{\xi}\right)\right\|+e^{-\frac{\alpha \rho}{\epsilon}}\|w\|\right) \leq C e^{-\frac{\alpha \rho}{\epsilon}} .
$$

We now estimate the terms on the right hand side of (3.23). Using (3.30) we have

$$
\begin{aligned}
& \left.\mid \int_{0}^{1} W_{\text {uиu }}\left(u^{\xi}\right) \sum_{h=1}^{N} u_{\xi_{h}}^{\xi} \dot{\xi}_{h} w \cdot w\right) d x \mid \\
& \leq C_{\epsilon} e^{-\frac{\alpha \rho}{\epsilon}} \int_{0}^{1}|w|^{2} d x=C_{\epsilon} e^{-\frac{\alpha \rho}{\epsilon}}\|w\|^{2} .
\end{aligned}
$$

From $\|w\|_{L^{\infty}} \leq \frac{C}{\epsilon^{\frac{1}{2}}}\|w\|_{W_{\epsilon}^{1,2}}$, (3.27) and (3.21) we have $\left\|N^{\xi}(w)\right\| \leq C\|w\|_{W_{\epsilon}^{1,2}}\|w\| \leq$ $e^{-\frac{\alpha \rho}{\epsilon}}\|w\|$ which implies

$$
\begin{aligned}
& \left|\left\langle L^{\xi} w, N^{\xi}(w)\right\rangle\right| \leq e^{-\frac{\alpha \rho}{2 \epsilon}}\left\|L^{\xi} w\right\|\|w\| \leq e^{-\frac{\alpha \rho}{2 \epsilon}}\left(\left\|L^{\xi} w\right\|^{2}+\|w\|^{2}\right), \\
& \left|\left\langle L^{\xi} w, \mathscr{F}\left(u^{\xi}\right)\right\rangle\right| \leq\left\|L^{\xi} w\right\|\left\|\mathscr{F}\left(u^{\xi}\right)\right\| \leq \frac{1}{4}\left\|L^{\xi} w\right\|^{2}+\left\|\mathscr{F}\left(u^{\xi}\right)\right\|^{2} .
\end{aligned}
$$

Finally we observe that Lemma 2.7 and $L^{\xi} u_{\xi_{h}}=-\mathscr{F}_{\xi_{h}}\left(u^{\xi}\right)$ together with (3.30) imply

$$
\begin{aligned}
& \left|\left\langle L^{\xi} w, \sum_{h=1}^{N} u_{\xi_{h}}^{\xi} \dot{\xi}_{h}\right\rangle\right| \leq\|w\| \sum_{h}\left\|\mathscr{F}_{\xi_{h}}\left(u^{\xi}\right)\right\|\left|\dot{\xi}_{h}\right| \\
& \leq C e^{-\frac{\alpha \rho}{\epsilon}}\|w\|\left(C\left\|\mathscr{F}\left(u^{\xi}\right)\right\|+e^{-\frac{\alpha \rho}{\epsilon}}\|w\|\right) \leq C e^{-\frac{\alpha \rho}{\epsilon}}\left(\left\|\mathscr{F}\left(u^{\xi}\right)\right\|^{2}+\|w\|^{2}\right) .
\end{aligned}
$$


From these estimates and (3.23) it follows

$$
\frac{1}{2} \frac{d}{d t}\left\langle w, L^{\xi} w\right\rangle \leq-\frac{1}{2}\left\|L^{\xi} w\right\|^{2}+2\left\|\mathscr{F}\left(u^{\xi}\right)\right\|^{2}+C e^{-\frac{\alpha \rho}{\epsilon}}\|w\|^{2} .
$$

Lemma 3.3. There exist positive constants $\nu$ and $D$ independent of $\xi \in \Xi_{\rho}$ and of $\epsilon>0$ such that $w \perp \operatorname{span}\left(\frac{u_{\xi_{1}}^{\xi}}{\left\|u_{\xi_{1}}^{\xi}\right\|}, \ldots, \frac{u_{\xi_{N}}^{\xi}}{\left\|u_{\xi_{N}}^{\xi}\right\|}\right)$ implies

$$
\begin{aligned}
& \mu^{*}\|w\|_{W_{\epsilon}^{1,2}}^{2} \leq\left\langle w, L^{\xi} w\right\rangle \leq D\|w\|_{W_{\epsilon}^{1,2}}^{2}, \\
& \left\langle w, L^{\xi} w\right\rangle \leq \nu\left\|L^{\xi} w\right\|^{2} \\
& \|w\| \leq \nu\left\|L^{\xi} w\right\| .
\end{aligned}
$$

Proof. We have $w=w^{\prime \prime}+w^{\perp}$ with $w^{\prime \prime} \in X^{\xi}$ and $w^{\perp} \in X^{\xi, \perp}$. From (3.12) and Lemma 2.9 it follows

$$
\begin{aligned}
w^{\prime \prime} & =\sum_{j}\left\langle w, \varphi_{j}^{\xi}\right\rangle \varphi_{j}^{\xi}=\sum_{j}\left\langle w, \eta_{j}^{\xi}\right\rangle \varphi_{j}^{\xi}, \\
w_{x}^{\prime \prime} & =\sum_{j}\left\langle w, \eta_{j}^{\xi}\right\rangle \varphi_{j, x}^{\xi} .
\end{aligned}
$$

This, Lemma 2.11 and Lemma 2.9 that imply $\left\|\varphi_{j, x}^{\xi}\right\|=\mathrm{O}\left(\frac{C}{\epsilon^{\frac{1}{2}}}\right)$, yields

$$
\begin{aligned}
& \left\|w^{\prime \prime}\right\| \leq e^{-\frac{\alpha \rho}{\epsilon}}\|w\| \leq e^{-\frac{\alpha \rho}{\epsilon}}\|w\|_{W_{\epsilon}^{1,2}}, \\
& \left\|w_{x}^{\prime \prime}\right\| \leq e^{-\frac{\alpha \rho}{\epsilon}}\|w\| \leq e^{-\frac{\alpha \rho}{\epsilon}}\|w\|_{W_{\epsilon}^{1,2}} .
\end{aligned}
$$

and in turn

$$
\begin{aligned}
& \left(1-e^{-\frac{\alpha \rho}{\epsilon}}\right)\|w\| \leq\left\|w^{\perp}\right\| \leq\|w\|, \\
& \left(1-e^{-\frac{\alpha \rho}{\epsilon}}\right)\|w\|_{W_{\epsilon}^{1,2}} \leq\left\|w^{\perp}\right\|_{W_{\epsilon}^{1,2}} \leq\left(1+e^{-\frac{\alpha \rho}{\epsilon}}\right)\|w\|_{W_{\epsilon}^{1,2} .}
\end{aligned}
$$

Let $\varphi_{j}^{\xi}$ and $\lambda_{j}^{\xi}, \lambda_{j}^{\xi} \leq \lambda_{j+1}^{\xi}, j=1, \ldots$ the eigenvectors and the corresponding eigenvalues of $L^{\xi}$. Then Proposition 2.1 yields

$$
\begin{aligned}
& \left(\lambda_{N+1}^{\xi}\right)^{2}\left\|w^{\perp}\right\|^{2} \leq\left\|L^{\xi} w^{\perp}\right\|^{2} \leq\left\|L^{\xi} w\right\|^{2}, \\
& \left\|L^{\xi} w^{\prime \prime}\right\|^{2} \leq e^{-\frac{\alpha \rho}{\epsilon}}\left\|w^{\prime \prime}\right\|^{2}
\end{aligned}
$$

This and (3.38) imply $(3.35) 3$ with $\nu=\sup _{\xi \in \Xi_{\rho}} \frac{1}{2 \lambda_{N+1}^{\xi}}$. The inequality (3.35) 2 follows from $(3.35)_{3}$. To complete the proof we note that Proposition 2.1 implies

$$
\mu\left\|w^{\perp}\right\|_{W_{\epsilon}^{1,2}}^{2}+\left\langle w^{\prime \prime}, L^{\xi} w^{\prime \prime}\right\rangle \leq\left\langle w, L^{\xi} w\right\rangle .
$$

The left inequality in $(3.35) 1$ follows from this (3.38) and $\left|\left\langle w^{\prime \prime}, L^{\xi} w^{\prime \prime}\right\rangle\right| \leq e^{-\frac{\alpha \rho}{\epsilon}}\|w\|_{W_{\epsilon}^{1,2}}^{2}$. The right inequality from $\left|W_{u u}\left(u^{\xi}\right)\right| \leq C$.

From Lemma 3.3 and (3.34) it follows

$$
\begin{aligned}
& \frac{1}{2} \frac{d}{d t}\left\langle w, L^{\xi} w\right\rangle \leq-\frac{1}{2}\left\|L^{\xi} w\right\|^{2}+2\left\|\mathscr{F}\left(u^{\xi}\right)\right\|^{2}+C_{\epsilon} e^{-\frac{\alpha \rho}{\epsilon}}\|w\|^{2} \\
& \leq-\frac{1}{4}\left\|L^{\xi} w\right\|^{2}+2\left\|\mathscr{F}\left(u^{\xi}\right)\right\|^{2} \leq-\frac{1}{4 \nu}\left\langle w, L^{\xi} w\right\rangle+2\left\|\mathscr{F}\left(u^{\xi}\right)\right\|^{2} .
\end{aligned}
$$

Which should be complemented by (3.29). 
Lemma 3.4. There exist constants $Q>0$ and $\beta>1$, independent of $\xi \in \Xi_{\rho}$ and $\epsilon \in\left(0, \epsilon_{0}\right]$, such that the condition

$$
\left\|w_{0}\right\|_{W_{\epsilon}^{1,2}} \leq Q\left\|\mathscr{F}\left(u^{\xi_{0}}\right)\right\|
$$

as long as $\xi(t) \in \Xi_{\rho}$, implies

$$
\|w(t)\|_{W_{\epsilon}^{1,2}} \leq \beta Q\left\|\mathscr{F}\left(u^{\xi(t)}\right)\right\| .
$$

Proof. Set $Q=4 \sqrt{\frac{2 \nu}{\mu^{*}}}, \beta=\sqrt{\frac{2 D}{\mu^{*}}+\frac{1}{2}}$ and $\bar{t}=2 \nu \ln \frac{4 D}{\mu^{*}}$ and observe that (3.35) implies $\beta>1$. From this and the condition $\left\|w_{0}\right\|_{W_{\epsilon}^{1,2}} \leq Q\left\|\mathscr{F}\left(u^{\xi_{0}}\right)\right\|$ it follows

$$
\|w(t)\|_{W_{\epsilon}^{1,2}} \leq \beta Q\left\|\mathscr{F}\left(u^{\xi(t)}\right)\right\|, \quad t \in\left[0, \min \left\{t^{*}, \bar{t}\right\}\right],
$$

for some $t^{*}>0$.This and (3.30) imply

$$
\left|\dot{\xi}_{h}\right| \leq C \epsilon^{\frac{1}{2}}\left\|\mathscr{F}\left(u^{\xi(t)}\right)\right\|, \quad t \in\left[0, \min \left\{t^{*}, \bar{t}\right\}\right],
$$

and therefore, from $(2.36))_{2}$ we obtain

$$
\begin{aligned}
& \left|\frac{d}{d t}\left\|\mathscr{F}\left(u^{\xi(t)}\right)\right\|\right| \leq \sum_{h=1}^{N}\left\|\mathscr{F}_{\xi_{h}}\left(u^{\xi(t)}\right)\right\|\left|\dot{\xi}_{h}(t)\right| \\
& \leq e^{-\frac{\alpha \rho}{\epsilon}}\left\|\mathscr{F}\left(u^{\xi(t)}\right)\right\|, \quad t \in\left[0, \min \left\{t^{*}, \bar{t}\right\}\right] .
\end{aligned}
$$

It follows

$$
\left(1-e^{-\frac{\alpha \rho}{\epsilon}} \bar{t}\right)\left\|\mathscr{F}\left(u^{\xi_{0}}\right)\right\| \leq\left\|\mathscr{F}\left(u^{\xi(t)}\right)\right\| \leq\left(1+2 e^{-\frac{\alpha \rho}{\epsilon}} \bar{t}\right)\left\|\mathscr{F}\left(u^{\xi_{0}}\right)\right\|, \quad t \in\left[0, \min \left\{t^{*}, \bar{t}\right\}\right] .
$$

This and (3.39) yield

$$
\frac{d}{d t}\left\langle w, L^{\xi} w\right\rangle+\frac{1}{2 \nu}\left\langle w, L^{\xi} w\right\rangle \leq 4\left(1+2 e^{-\frac{\alpha \rho}{\epsilon}} \bar{t}\right)^{2}\left\|\mathscr{F}\left(u^{\xi_{0}}\right)\right\|^{2}, \quad t \in\left[0, \min \left\{t^{*}, \bar{t}\right\}\right],
$$

which implies

$$
\left\langle w, L^{\xi} w\right\rangle \leq\left\langle w_{0}, L^{\xi} w_{0}\right\rangle e^{-\frac{t}{2 \nu}}+8 \nu\left(1-e^{-\frac{t}{2 \nu}}\right)\left(1+2 e^{-\frac{\alpha \rho}{\epsilon}} \bar{t}\right)^{2}\left\|\mathscr{F}\left(u^{\xi_{0}}\right)\right\|^{2}, \quad t \in\left[0, \min \left\{t^{*}, \bar{t}\right\}\right] .
$$

This and Lemma 3.3 imply

$$
\begin{aligned}
& \mu^{*}\|w(t)\|_{W_{\epsilon}^{1,2}}^{2} \leq\left\langle w, L^{\xi} w\right\rangle \leq e^{-\frac{t}{2 \nu}} D\left\|w_{0}\right\|_{W_{\epsilon}^{1,2}}^{2}+8 \nu\left(1+2 e^{-\frac{\alpha \rho}{\epsilon}} \bar{t}\right)^{2}\left\|\mathscr{F}\left(u^{\xi_{0}}\right)\right\|^{2} \\
& \Rightarrow \quad\|w(t)\|_{W_{\epsilon}^{1,2}}^{2} \leq \frac{1}{\mu^{*}}\left(e^{-\frac{t}{2 \nu}} D Q^{2}+8 \nu\left(1+2 e^{-\frac{\alpha \rho}{\epsilon}} \bar{t}\right)^{2}\right)\left\|\mathscr{F}\left(u^{\xi_{0}}\right)\right\|^{2} \\
& \leq \frac{e^{-\frac{t}{2 \nu}} D Q^{2}+8 \nu\left(1+2 e^{-\frac{\alpha \rho}{\epsilon}} \bar{t}\right)^{2}}{\mu^{*}\left(1-e^{-\frac{\alpha \rho}{\epsilon}} \bar{t}\right)^{2}}\left\|\mathscr{F}\left(u^{\xi(t)}\right)\right\|^{2}, \quad t \in\left[0, \min \left\{t^{*}, \bar{t}\right\}\right],
\end{aligned}
$$

where we have also used (3.42). Assume that $\epsilon>0$ is so small that $\frac{\left(1+2 e^{-\frac{\alpha \rho}{\epsilon}} \bar{t}\right)^{2}}{\left(1-e^{-\frac{\alpha \rho}{\epsilon}} \bar{t}\right)^{2}} \leq 2$, then (3.43) implies

$$
\|w(t)\|_{W_{\epsilon}^{1,2}}^{2} \leq \frac{2 D Q^{2}+16 \nu}{\mu^{*}}\left\|\mathscr{F}\left(u^{\xi(t)}\right)\right\|^{2}=\beta^{2} Q^{2}\left\|\mathscr{F}\left(u^{\xi(t)}\right)\right\|^{2}, \quad t \in\left[0, \min \left\{t^{*}, \bar{t}\right\}\right]
$$


and

$$
\|w(\bar{t})\|_{W_{\epsilon}^{1,2}}^{2} \leq\left(2 \frac{e^{-\frac{\bar{t}}{2 \nu}}}{\mu^{*}} D Q^{2}+\frac{16 \nu}{\mu^{*}}\right)\left\|\mathscr{F}\left(u^{\xi(\bar{t})}\right)\right\|^{2}=Q^{2}\left\|\mathscr{F}\left(u^{\xi(\bar{t})}\right)\right\|^{2} .
$$

From (3.44) It follows that $\min \left\{t^{*}, \bar{t}\right\}=\bar{t}$ and therefore that (3.43) is valid for $t \in[0, \bar{t}]$. This and (3.45) imply that we can repeat what we have said above for $t \in[0, \bar{t}]$ for the interval $[\bar{t}, 2 \bar{t}]$ and so on as long as $\xi(t) \in \Xi_{\rho}$. This concludes the proof.

We are now in the position of completing the proof of Theorem 1.3. Indeed (1.20) follows by inserting the estimate (3.40) for $w$ into (3.29), by observing that Lemma 2.7 implies

$$
\begin{aligned}
& \left\|\mathscr{F}\left(u^{\xi}\right)\right\| \leq C e^{-\frac{\alpha \rho}{\epsilon}} \max _{h, \pm} \mathscr{E}_{h}^{ \pm} \frac{1}{2}, \\
& \left\|\mathscr{F}_{\xi_{j}}\left(u^{\xi}\right)\right\| \leq \frac{C}{\epsilon} \max _{h, \pm} \mathscr{E}_{h}^{ \pm}, \quad j=1, \ldots, N .
\end{aligned}
$$

and by recalling (2.33). This conclude the proof of the theorem.

\subsection{The proof of Lemma 3.2 .}

Proof. 1. For $\xi \in \Xi_{\rho}$, we have

$$
\begin{aligned}
& u^{\xi}\left(\xi_{j}\right)=\bar{u}_{j}(0)+\mathrm{O}\left(e^{-\frac{\alpha \rho}{\epsilon}}\right), \\
& u^{\xi}\left(\hat{\xi}_{j-1}\right)=a_{j}+\mathrm{O}\left(e^{-\frac{\alpha \rho}{\epsilon}}\right), \quad\left(\hat{\xi}_{h}=\frac{\xi_{h+1}+\xi_{h}}{2}\right), \\
& u^{\xi}\left(\hat{\xi}_{j}\right)=a_{j+1}+\mathrm{O}\left(e^{-\frac{\alpha \rho}{\epsilon}}\right) .
\end{aligned}
$$

This follows from (2.29), (1.8) and Lemma 2.6.

2. Assume that there is $1 \leq j \leq N$ such that $\xi, \zeta \in \Xi_{\rho}$ satisfy

$$
\zeta_{h} \notin\left(\hat{\xi}_{j-1}, \hat{\xi}_{j}\right), \quad h \neq j, h=1, \ldots, N,
$$

then there is $\bar{m}_{1}>0$ independent of $\epsilon>0$ such that

$$
\left\|u^{\zeta}-u^{\xi}\right\|_{L^{\infty}} \geq \bar{m}_{1} .
$$

To prove this we note that (3.47) implies $u^{\zeta}\left(\xi_{j}\right)=a_{h}+\mathrm{O}\left(e^{-\frac{\alpha \rho}{\epsilon}}\right)$ for some $1 \leq h \leq N$ that together with (3.46) and

$$
\left\{\bar{u}_{i}(s): s \in \mathbb{R}\right\} \cap A=\emptyset, \quad(A=\{W=0\}) .
$$

that follows from the minimality of $\bar{u}_{i}, i=1, \ldots, N$, imply $u^{\zeta}\left(\xi_{j}\right)-u^{\xi}\left(\xi_{j}\right)=a_{h}-\bar{u}_{j}(0)+$ $\mathrm{O}\left(e^{-\frac{\alpha \rho}{\epsilon}}\right) \neq 0$ where we have assumed $\epsilon>0$ sufficiently small. This implies (3.48) with $\bar{m}_{1}=\frac{1}{2}\left|a_{h}-\bar{u}_{j}(0)\right|$.

3. Assume now that $\xi, \zeta$ are such that there is no $j$ that satisfies (3.47). This implies that, for each $j$ there is one and exactly one $\zeta_{h_{j}} \in\left(\hat{\xi}_{j-1}, \hat{\xi}_{j}\right)$. There are three different possibilities

a) there is $j$ such that $a_{h_{j}} \neq a_{j}$ and $a_{h_{j}+1} \neq a_{j+1}$.

b) there is $j$ such that either $a_{h_{j}}=a_{j}$ and $a_{h_{j}+1} \neq a_{j+1}$ or $a_{h_{j}} \neq a_{j}$ and $a_{h_{j}+1}=a_{j+1}$. 
c) $a_{h_{j}}=a_{j}$ and $a_{h_{j}+1}=a_{j+1}$ for all $j$.

In case a), for small $\epsilon>0$, we have

$$
\begin{aligned}
& \zeta_{h_{j}} \leq \xi_{j} \Rightarrow u^{\zeta}\left(\xi_{j}+d\right)-u^{\xi}\left(\xi_{j}+d\right)=a_{h_{j}+1}-a_{j+1}+\mathrm{O}\left(e^{-\frac{\alpha \rho}{\epsilon}}\right) \neq 0, \\
& \zeta_{h_{j}} \geq \xi_{j} \Rightarrow u^{\zeta}\left(\xi_{j}-d\right)-u^{\xi}\left(\xi_{j}-d\right)=a_{h_{j}}-a_{j}+\mathrm{O}\left(e^{-\frac{\alpha \rho}{\epsilon}}\right) \neq 0,
\end{aligned}
$$

where $d=\frac{\rho}{4 \max _{i} \mu_{i}}$. It follows that (3.48) holds also in this case with $\bar{m}_{1}=\frac{1}{2}\left(\mid a_{h_{j}+1}-\right.$ $\left.a j+1|+| a_{h_{j}}-a j \mid\right)$.

Case b). We only discuss the case $a_{h_{j}}=a_{j}$, the argument for the case $a_{h_{j}+1}=a_{j+1}$ is analogous. If $\zeta_{h_{j}} \leq \xi_{j}$ arguing as in a) we again obtain (3.48). Therefore we assume $\zeta_{h_{j}}>\xi_{j}$. We observe that the minimality of $\bar{u}_{h_{j}}$ and $\bar{u}_{j}$ together with $a_{h_{j}}=a_{j}$ and $a_{h_{j}+1} \neq a_{j+1}$ imply the existence of $\delta>0$ such that

$$
\left|\bar{u}_{h_{j}}(s)-a_{j+1}\right| \geq \delta, \quad s \in \mathbb{R} .
$$

it follows, provided $\epsilon>0$ is small

$$
\left|u^{\zeta}\left(\xi_{j}+d\right)-a_{j+1}-\left(u^{\xi}\left(\xi_{j}+d\right)-a_{j+1}\right)\right|=\left|u^{\zeta}\left(\xi_{j}+d\right)-a_{j+1}\right|+\mathrm{O}\left(e^{-\frac{\alpha \rho}{\epsilon}}\right) \geq \frac{\delta}{2}
$$

and we conclude that (3.48) holds also in case b) with $\bar{m}_{1}=\frac{\delta}{2}$.

Case c). In this case we necessarily have $h_{j}=j, 1 \leq j \leq N$ and in turn $\bar{u}_{h_{j}}=\bar{u}_{j}$. The minimality of $\bar{u}_{j}$ implies

$$
\bar{u}_{j}(s) \neq \bar{u}_{j}(s+\tau), \quad s \in \mathbb{R}, \tau \neq 0,
$$

and (3.49) yields $\bar{u}_{j}(0) \notin\left\{a_{j}, a_{j+1}\right\}$. It follows that, given $\omega>0$, there is $\bar{m}_{1}(\omega)>0$ such that

$$
\left|\bar{u}_{j}(s)-\bar{u}_{j}(0)\right| \geq \bar{m}_{1}(\omega), \quad|s| \geq \omega .
$$

This and Lemma 2.6 yield

$$
\left|\zeta_{j}-\xi_{j}\right| \geq \epsilon \omega \Rightarrow\left|u^{\zeta}\left(\xi_{j}\right)-u^{\xi}\left(\xi_{j}\right)\right| \geq \bar{m}_{1}(\omega)+\mathrm{O}\left(e^{-\frac{\alpha \rho}{\epsilon}}\right) .
$$

From 1, 2, 3 we conclude that

$$
|\zeta-\xi| \geq \epsilon \omega \Rightarrow\left\|u^{\zeta}-u^{\xi}\right\|_{L^{\infty}} \geq \frac{1}{2} \bar{m}_{1}(\omega)
$$

That establishes (3.5) 1 with $m_{1}(\omega)=\frac{1}{2} \bar{m}_{1}(\omega)$ and $\omega_{1}$ such that $m_{1}(\omega) \leq \bar{m}_{1}$ for $\omega \in\left(0, \omega_{1}\right]$. This and $\left|u_{x}^{\xi}(x)\right| \leq \frac{C}{\epsilon}$ that follows from (2.33) 3 imply

$$
|\zeta-\xi| \geq \epsilon \omega \Rightarrow\left\|u^{\zeta}-u^{\xi}\right\| \geq C\left(m_{1}(\omega)\right)^{\frac{3}{2}} \epsilon^{\frac{1}{2}}
$$

that yields $(3.6) 1$.

4. Proof of (3.6) 2. Fix a number $\omega_{0}>0$. Then, for $|\zeta-\xi| \leq \omega_{0} \epsilon$, the estimates in Lemma 2.6 apply both to $u^{\xi}$ and $u^{\zeta}$ and we have

$$
\begin{aligned}
& u^{\zeta}(x)-u^{\xi}(x)=\int_{0}^{1} u_{\xi}^{\xi+t(\zeta-\xi)} d t \cdot(\zeta-\xi) \\
& =-\frac{1}{\epsilon} \sum_{j} \int_{0}^{1} \bar{u}_{j}^{\prime}\left(\frac{x-\xi_{j}-t\left(\zeta_{j}-\xi_{j}\right)}{\epsilon}\right)\left(\zeta_{j}-\xi_{j}\right) d t+\sum_{j} \mathrm{O}\left(e^{-\frac{\alpha \rho}{\epsilon}}\right)\left(\zeta_{j}-\xi_{j}\right) .
\end{aligned}
$$


Note that

$$
\bar{u}_{j}^{\prime}\left(\frac{x-\xi_{j}-t\left(\zeta_{j}-\xi_{j}\right)}{\epsilon}\right)=\bar{u}_{j}^{\prime}\left(\frac{x-\xi_{j}}{\epsilon}\right)-\frac{t}{\epsilon} \int_{0}^{1} \bar{u}_{j}^{\prime \prime}\left(\frac{x-\xi_{j}-\tau t\left(\zeta_{j}-\xi_{j}\right)}{\epsilon}\right) d \tau\left(\zeta_{j}-\xi_{j}\right)
$$

implies

$$
\begin{aligned}
& u^{\zeta}(x)-u^{\xi}(x)=-\frac{1}{\epsilon} \sum_{j} \bar{u}_{j}^{\prime}\left(\frac{x-\xi_{j}}{\epsilon}\right)\left(\zeta_{j}-\xi_{j}\right) \\
& +\int_{0}^{1} \frac{t}{\epsilon^{2}} \sum_{j} \int_{0}^{1} \bar{u}_{j}^{\prime \prime}\left(\frac{x-\xi_{j}-\tau t\left(\zeta_{j}-\xi_{j}\right)}{\epsilon}\right) d t d \tau\left(\zeta_{j}-\xi_{j}\right)^{2}+\sum_{j} \mathrm{O}\left(e^{-\frac{\alpha \rho}{\epsilon}}\right)\left(\zeta_{j}-\xi_{j}\right)
\end{aligned}
$$

Arguing as in the proof of Lemma 2.6 we find

$$
\frac{1}{\epsilon^{2}} \int_{0}^{1} \bar{u}_{j}^{\prime}\left(\frac{x-\xi_{j}}{\epsilon}\right) \cdot \bar{u}_{i}^{\prime}\left(\frac{x-\xi_{i}}{\epsilon}\right) d x=\frac{\delta_{i j}}{\epsilon} \bar{q}_{j}^{2}+\mathrm{O}\left(e^{-\frac{\alpha \rho}{\epsilon}}\right),
$$

where $\bar{q}_{j}^{2}=\int_{\mathbb{R}}\left|\bar{u}_{j}(s)\right|^{2} d s$. From (3.53) we obtain

$$
\begin{aligned}
& \left\|\frac{1}{\epsilon} \sum_{j} \bar{u}_{j}^{\prime}\left(\frac{x-\xi_{j}}{\epsilon}\right)\left(\zeta_{j}-\xi_{j}\right)\right\| \geq\left(\frac{\bar{q}_{m}}{\epsilon^{\frac{1}{2}}}+\mathrm{O}\left(e^{-\frac{\alpha \rho}{\epsilon}}\right)\right)|\zeta-\xi|, \\
& \left\|\frac{1}{\epsilon} \sum_{j} \bar{u}_{j}^{\prime}\left(\frac{x-\xi_{j}}{\epsilon}\right)\left(\zeta_{j}-\xi_{j}\right)\right\| \leq\left(\frac{\bar{q}_{M}}{\epsilon^{\frac{1}{2}}}+\mathrm{O}\left(e^{-\frac{\alpha \rho}{\epsilon}}\right)\right)|\zeta-\xi|,
\end{aligned}
$$

where $\bar{q}_{m}$ and $\bar{q}_{M}$ are respectively the minimum and the maximum of the $\bar{q}_{j}$.

From $\left|x-\xi_{j}-\tau t\left(\zeta_{j}-\xi_{j}\right)\right| \geq\left|x-\xi_{j}\right|-\left|\zeta_{j}-\xi_{j}\right|$, (1.8) and the assumption $|\zeta-\xi| \leq \omega_{0} \epsilon$ it follows

$$
\left|\bar{u}_{j}^{\prime \prime}\left(\frac{x-\xi_{j}-\tau t\left(\zeta_{j}-\xi_{j}\right)}{\epsilon}\right)\right| \leq C e^{-\frac{\mu_{j}}{\epsilon}\left|x-\xi_{j}\right|} e^{\mu_{j} \omega_{0}} \leq C e^{-\frac{\mu_{j}}{\epsilon}\left|x-\xi_{j}\right|},
$$

Therefore if $I(x)=\sum_{j} I_{j}(x)$ is the integral term in (3.52) we have

$$
\begin{aligned}
& |\zeta-\xi| \leq \omega_{0} \epsilon \Rightarrow \\
& \left\|I_{j}\right\|^{2} \leq \frac{1}{\epsilon^{4}} C \int_{0}^{1} e^{-\frac{2 \mu_{j}}{\epsilon}\left|x-\xi_{j}\right|} d x\left(\zeta_{j}-\xi_{j}\right)^{4} \\
& \leq \frac{C}{\epsilon^{3}}\left(\zeta_{j}-\xi_{j}\right)^{4} \leq \frac{C \omega_{0}^{2}}{\epsilon}\left(\zeta_{j}-\xi_{j}\right)^{2} \\
& \|I\| \leq \sum_{j}\left\|I_{j}\right\| \leq \frac{C \omega_{0}}{\epsilon^{\frac{1}{2}}}|\zeta-\xi| .
\end{aligned}
$$

This (3.52) and (3.54) yield

$$
\begin{aligned}
& |\zeta-\xi| \leq \omega_{0} \epsilon \Rightarrow \\
& \left\|u^{\zeta}-u^{\xi}\right\| \geq \frac{1}{\epsilon^{\frac{1}{2}}}\left(\bar{q}_{m}-C \omega+\mathrm{O}\left(e^{-\frac{\alpha \rho}{\epsilon}}\right)|\zeta-\xi|,\right. \\
& \left\|u^{\zeta}-u^{\xi}\right\| \leq \frac{1}{\epsilon^{\frac{1}{2}}}\left(\bar{q}_{M}+C \omega+\mathrm{O} e^{-\frac{\alpha \rho}{\epsilon}}\right)|\zeta-\xi|,
\end{aligned}
$$


and therefore, for $\omega_{0}=\frac{\bar{q}_{m}}{4 C}$, we have

$$
\begin{aligned}
& |\zeta-\xi| \leq \omega_{0} \epsilon \quad \Rightarrow \\
& \frac{\bar{q}_{m}}{2 \epsilon^{\frac{1}{2}}}|\zeta-\xi| \leq\left\|u^{\zeta}-u^{\xi}\right\| \leq \frac{2 \bar{q}_{M}}{\epsilon^{\frac{1}{2}}}|\zeta-\xi| .
\end{aligned}
$$

This concludes the proof of $(3.6)_{2}$. The proof of (3.7) is similar.

5. Proof of $(3.5)_{2}$. The right inequality follows immediately from (3.51) and the boundedness of $\bar{u}_{j}^{\prime}, j=1, \ldots, N$. To prove the other inequality fix a number $s_{a}>0$ that satisfies

$$
\left|\bar{u}_{i}\left(s_{a}\right)-\bar{u}_{i}\left(-s_{a}\right)\right| \geq \frac{1}{2} \min _{j}\left|a_{j+1}-a_{j}\right|, \quad i=1, \ldots, N .
$$

Then there is $s_{i} \in\left[-s_{a}, s_{a}\right]$ such that

$$
\left|\bar{u}_{i}^{\prime}\left(s_{i}\right)\right| \geq k_{a}=\frac{1}{4 s_{a}} \min _{j}\left|a_{j+1}-a_{j}\right| .
$$

Assume that $h$ satisfies $\left|\zeta_{h}-\xi_{h}\right|=\max _{j}\left|\zeta_{j}-\xi_{j}\right|$ and note that, for $x=\xi_{h}+\epsilon s_{h}$ we have

$$
\begin{aligned}
& \left|\sum_{j} \bar{u}_{j}^{\prime}\left(\frac{\xi_{h}-\xi_{j}+\epsilon s_{h}}{\epsilon}\right)\left(\zeta_{j}-\xi_{j}\right)\right| \\
& \geq\left(k_{a}-\sum_{j \neq h}\left|\bar{u}_{j}^{\prime}\left(\frac{\xi_{h}-\xi_{j}+\epsilon s_{h}}{\epsilon}\right)\right|\right)\left|\zeta_{h}-\xi_{h}\right| \geq \frac{k_{a}}{2 N^{\frac{1}{2}}}|\zeta-\xi|,
\end{aligned}
$$

where we have observed that $\left|\bar{u}_{j}^{\prime}\left(\frac{\xi_{h}-\xi_{j}+\epsilon s_{h}}{\epsilon}\right)\right|=\mathrm{O}\left(e^{-\frac{\alpha \rho}{\epsilon}}\right)$, if $j \neq h$. On the other hand the boundedness of $\bar{u}_{j}^{\prime \prime}(s)$ implies

$$
\begin{aligned}
& \left|I_{j}\left(\xi_{h}+\epsilon s_{h}\right)\right| \leq \frac{C}{\epsilon^{2}}\left|\zeta_{j}-\xi_{j}\right|^{2} \Rightarrow \\
& \left|I\left(\xi_{h}+\epsilon s_{h}\right)\right| \leq \frac{C}{\epsilon^{2}}|\zeta-\xi|^{2} \leq \frac{k_{a}}{4 \epsilon N^{\frac{1}{2}}}|\zeta-\xi|,
\end{aligned}
$$

where we have used the hypothesis $|\zeta-\xi| \leq \epsilon \omega_{1}$ and assumed $\omega_{1} \leq \frac{k_{0}}{4 N^{\frac{1}{2}}}$. This , (3.52), (3.57) and a proper definition of $C_{ \pm}^{1}$ conclude the proof of the estimate (3.5) 2 .

\section{On the asymptotic behavior of $\bar{u}$.}

Lemma 4.1. Assume that $W: \mathbb{R}^{m} \rightarrow \mathbb{R}$ satisfies $\mathbf{H}_{2}$ and $\bar{u}:(0,+\infty) \rightarrow \mathbb{R}^{m}$ is a solution of (1.1) that converges to some $a \in A: \lim _{s \rightarrow+\infty} \bar{u}(s)=a$. Then there exists

$$
\lim _{s \rightarrow+\infty} \frac{\bar{u}(s)-a}{|\bar{u}(s)-a|}=z,
$$

and $z$ is an eigenvector of the matrix $W_{u u}(a)$. Moreover there are positive constants $\bar{K}, K^{+}$and $\mu^{+}>\mu\left(\mu^{2}\right.$ the eigenvalue of $W_{u u}(a)$ associated to $\left.z\right)$ and a smooth map $w:(0,+\infty) \rightarrow \mathbb{R}^{m}$ such that

$$
\begin{aligned}
& \bar{u}(s)-a=\bar{K} z e^{-\mu s}+w(s), \quad s>0, \\
& |w(s)|,\left|D^{k} w(s)\right| \leq K^{+} e^{-\mu^{+} s}, \quad s>0, \quad k=1,2,3
\end{aligned}
$$

A similar statements applies when $\bar{u}$ converges to a as $s \rightarrow-\infty$. 
Proof. 1. From the assumptions on $\bar{u}$ it follows that $\bar{u}$ lies on the stable manifold of $a$. This implies that, modulus a linear change of coordinates, we can assume that $a=0$ and $\bar{u}$ is a solution of

$$
\dot{u}=-D(\mu) u+f(u),
$$

where $f$ is a smooth maps that satisfies $|f(u)| \leq C_{f}|u|^{2}$ for some $C_{f}>0$ and $D(\mu)$ is the diagonal matrix whose elements $\mu_{1}, \ldots, \mu_{m}$ are the square roots of the eigenvalues $\mu_{1}^{2}, \ldots, \mu_{m}^{2}$ of $W_{u u}(a)$ and $u=\left(u_{1}, \ldots, u_{m}\right)$ is the vector of the components of $u$ on the orthonormal basis composed by the eigenvectors $z_{1}, \ldots, z_{m}$ associated to $\mu_{1}^{2}, \ldots, \mu_{m}^{2}$. With the change of variable $u=|u| \nu, r=|u|$ (4.1) transforms in the system

$$
\begin{aligned}
& \dot{\nu}=-D(\mu) \nu+(\nu \cdot D(\mu) \nu) \nu+\frac{f(r \nu)}{r}-\left(\nu \cdot \frac{f(r \nu)}{r}\right) \nu, \\
& \dot{r}=-(\nu \cdot D(\mu) \nu) r+f(r \nu) \cdot \nu .
\end{aligned}
$$

2. There exists $s_{0}>0$ such that

$$
r(s) \leq r\left(s_{0}\right) e^{-\frac{\mu_{m}}{2}\left(s-s_{0}\right)}, \quad s \geq s_{0},\left(\mu_{m}=\min _{h} \mu_{h}\right) .
$$

Since $\nu \cdot D(\mu) \nu \geq \mu_{m},(\underline{4.2})_{2}$ implies $\dot{r} \leq-\mu_{m} r+C_{f} r^{2}$. This and $\lim _{s \rightarrow+\infty} r(s)=0$ yield (4.3).

3. For $\delta>0$ small set $\mathscr{K}_{\delta}=\left\{\nu \in \mathbb{S}^{m-1}: \nu \notin \cup_{h} B_{\delta}\left(z_{h}\right)\right\}$ and define $V: \mathbb{S}^{m-1} \rightarrow \mathbb{R}$, $V(\nu)=\frac{1}{2} \nu \cdot D(\mu) \nu$. Then there are $c_{\delta}>0$ and $r_{\delta}>0$ such that

$$
\dot{V}(\nu) \leq-c_{\delta}, \quad \nu \in \mathscr{K}_{\delta}, r \leq r_{\delta} .
$$

We have

$$
\dot{V}(\nu)=\nu \cdot D(\mu) \dot{\nu}=-|D(\mu) \nu|^{2}+(\nu \cdot D(\mu) \nu)^{2}+F(r, \nu),
$$

where $|F(r, \nu)| \leq C_{f}^{\prime} r$, for some $C_{f}^{\prime}>0$. We have $(\nu \cdot D(\mu) \nu)^{2} \leq|D(\mu) \nu|^{2}$ with the sign of strict inequality unless $\nu \in\left\{z_{1}, \ldots, z_{m}\right\}$. Therefore $\nu \in \mathscr{K}_{\delta}$ implies $-|D(\mu) \nu|^{2}+(\nu$. $D(\mu) \nu)^{2} \leq-\hat{c}_{\delta}$ for some $\hat{c}_{\delta}>0$ and (4.5) yields

$$
\dot{V}(\nu) \leq-\hat{c}_{\delta}+C_{f}^{\prime} r \leq-\frac{\hat{c}_{\delta}}{2}=-c_{\delta}, \quad r \leq r_{\delta}=\frac{\hat{c}_{\delta}}{2 C_{f}^{\prime}} .
$$

4. The existence of a sequence $s_{n} \rightarrow+\infty$ such that

$$
\lim _{n \rightarrow+\infty} \nu\left(s_{n}\right)=\bar{\nu} \notin\left\{z_{1}, \ldots, z_{m}\right\},
$$

leads to a contradiction.

Fix $\delta \in\left(0, \bar{\delta}=\min _{h}\left|\bar{\nu}-z_{h}\right|\right)$ so small that

$$
\begin{aligned}
& V(\bar{\nu})>V_{\delta}^{-}=\max \left\{V(\nu): \nu \in \mathbb{S}^{m-1} \cap\left(\cup_{V\left(z_{h}\right)<V(\bar{\nu})} \bar{B}_{\delta}\left(z_{h}\right)\right)\right\}, \\
& V(\bar{\nu})<V_{\delta}^{+}=\min \left\{V(\nu): \nu \in \mathbb{S}^{m-1} \cap\left(\cup_{V\left(z_{h}\right)>V(\bar{\nu})} \bar{B}_{\delta}\left(z_{h}\right)\right)\right\} .
\end{aligned}
$$

this and (4.4) imply

$$
\dot{V}(\nu(s)) \leq-c_{\delta}, \quad \text { if } V(\nu(s)) \in\left[V_{\delta}^{-}, V_{\delta}^{+}\right], r(s) \leq r_{\delta} .
$$


This contradicts (4.6) and (4.7) that imply

$$
V\left(\nu\left(s_{n}\right)\right) \in\left[V_{\delta}^{-}, V_{\delta}^{+}\right], \quad n \geq \bar{n} .
$$

for some $\bar{n}$. This concludes the proof of the first part of the lemma. Indeed the compactness of $\mathbb{S}^{m-1}$ and 4 . implies that for each sequence $s_{n} \rightarrow+\infty$ there is a subsequence (still denoted $\left\{s_{n}\right\}$ ) such that

$$
\lim _{n \rightarrow+\infty} \nu\left(s_{n}\right)=z \in\left\{ \pm z_{1}, \ldots, \pm z_{m}\right\}
$$

and (4.8) implies that the limit $z$ is independent from the sequence.

From the existence of the limit

$$
\lim _{s \rightarrow+\infty} \frac{\bar{u}(s)-a}{|\bar{u}(s)-a|}=z,
$$

and the smoothness of $W$ it follows that, for $s \geq s_{0}$, for some $s_{0}>0$, it results

$$
\bar{u}(s)-a=r(s) z+v(r(s)),
$$

where $v:\left(0, r_{0}\right] \rightarrow \mathbb{R}^{m}, r_{0}>0$ a small number, is a smooth map that satisfies

$$
\begin{aligned}
& v(r) \cdot z=0, r \in\left[0, r_{0}\right], \\
& |v(r)| \leq C r^{2}, r \in\left[0, r_{0}\right] .
\end{aligned}
$$

From (4.10) we have

$$
|\dot{\bar{u}}|^{2}=|\dot{r}|^{2}\left(1+\left|v^{\prime}(r)\right|^{2}\right) .
$$

From this, conservation of energy: $|\dot{\bar{u}}|^{2}=2 W(\bar{u})$, and (4.10) we get

$$
|\dot{r}|^{2}\left(1+\left|v^{\prime}(r)\right|^{2}\right)=2 W(a+r z+v(r)) .
$$

Since $W(a)=W_{u}(a)=0$ it follows that, in a neighborhood of $a$, we have $W(u)=$ $\frac{1}{2} W_{u u}(a)(u-a) \cdot(u-a)+U(u-a)$ where $U$ is a smooth map defined in a neighborhood of $0 \in \mathbb{R}^{m}$ and $U(q)=\mathrm{O}\left(|q|^{3}\right)$. On the basis of these observations, and possibly after a renumbering of the coodinates, we can write

$$
2 W(a+r z+v(r))=\mu^{2} r^{2}+\sum_{h>1}^{m} \mu_{h}^{2} v_{h}^{2}(r)+2 U(r z+v(r)),
$$

where $\mu_{h}^{2}, 1 \leq h \leq m$ are the eigenvalues of $W_{u u}\left(a_{j+1}\right), \mu=\mu_{1}, z=z_{1}$ and $v_{h}$ is the projection of $v$ on the eigenvector $z_{h}$. Using (4.12) in (4.11) after taking the square root we see that $r(s)$ solves the equation

$$
\dot{r}=-\mu r+\left(\mu r-\left(\frac{\mu^{2} r^{2}+\sum_{h>1}^{m} \mu_{h}^{2} v_{h}^{2}(r)+2 U(r z+v(r))}{1+\left|v^{\prime}(r)\right|^{2}}\right)^{\frac{1}{2}}\right)=-\mu r+g(r)
$$

and from the properties of $v$ and $U$ we see that $g$ is a smooth function that satisfies

$$
|g(r)| \leq C r^{2}, r \leq r_{0}
$$


Fix $\bar{s} \in\left(s_{0},+\infty\right)$ and set $\bar{r}=r(\bar{s})$ and $s=\tau+\bar{s}$. We look at (4.13) as a weakly nonlinear equation and try to determine $\rho(\tau)=r(\tau+\bar{s})$ for $s \geq \bar{s}$ as a solution of

$$
\rho(\tau)=\bar{r} e^{-\mu \tau}+e^{-\mu \tau} \int_{0}^{\tau} e^{\mu t} g(\rho(t)) d t
$$

Set

$$
R_{\bar{r}}=\left\{\rho:[0,+\infty) \rightarrow \mathbb{R}: \rho(\tau) \leq 2 \bar{r} e^{-\mu \tau}\right\} .
$$

$R_{\bar{r}}$ with the distance $d(\rho, \hat{\rho})=\sup _{\tau \in[0,+\infty)}|\rho(\tau)-\hat{\rho}(\tau)| e^{\mu \tau}$ is a complete metric space. We now show that for $\bar{r}$ small, that is for each $\bar{s}$ sufficiently large, the right hand side of (4.15) defines a contraction map $\rho \stackrel{\mathcal{K}_{\bar{\zeta}}}{\rightarrow}$ on $R_{\bar{r}}$ and therefore it results

$$
r(\tau+\bar{s})=\rho^{*}(\tau), \tau \in[0,+\infty),
$$

where $\rho^{*} \in R_{\bar{r}}$ is the fixed point of $\mathcal{K}_{\bar{r}}$. From the definition of $\mathcal{K}_{\bar{r}}$ we have

$$
|\tilde{\rho}(\tau)| \leq \bar{r} e^{-\mu \tau}+e^{-\mu \tau} C \bar{r}^{2} \int_{0}^{\infty} e^{\mu t} e^{-2 \mu t} d t=\left(\bar{r}+\frac{C}{\mu} \bar{r}^{2}\right) e^{-\mu \tau}
$$

and therefore we have that $\tilde{\rho} \in R_{\bar{r}}$ provided

$$
\bar{r} \leq \frac{\mu}{C}
$$

We also have

$$
\left|\tilde{\rho}_{1}(\tau)-\tilde{\rho}_{2}(\tau)\right| \leq e^{-\mu \tau} \int_{0}^{\tau} e^{\mu t}\left|g\left(\rho_{1}(t)\right)-g\left(\rho_{2}(t)\right)\right| d t,
$$

and, using also $g^{\prime}(r) \leq C_{1} r$,

$$
\begin{aligned}
& \left|g\left(\rho_{1}(t)\right)-g\left(\rho_{2}(t)\right)\right| \\
& \left.=\left|\int_{0}^{1} g^{\prime}\left(\rho_{2}(t)+\theta\left(\rho_{1}(t)-\rho_{2}(t)\right)\right) d \theta\right| \mid \rho_{1}(t)-\rho_{2}(t)\right) \mid \\
& \left.\leq 2 C_{1} \bar{r} e^{-\mu t} \mid \rho_{1}(t)-\rho_{2}(t)\right) \mid .
\end{aligned}
$$

It follows

$$
\begin{aligned}
& \left.\left|\tilde{\rho}_{1}(\tau)-\tilde{\rho}_{2}(\tau)\right| \leq e^{-\mu \tau} 2 C_{1} \bar{r} \int_{0}^{\tau} e^{\mu t} e^{-\mu t} \mid \rho_{1}(t)-\rho_{2}(t)\right) \mid d t \\
& \left.\leq e^{-\mu \tau} 3 C_{1} \bar{r} \int_{0}^{\infty} e^{-\mu t} \sup _{\sigma \geq 0} e^{\mu \sigma} \mid \rho_{1}(\sigma)-\rho_{2}(\sigma)\right) \mid d t \\
& =e^{-\mu \tau} 2 C_{1} \bar{r} d\left(\rho_{1}, \rho_{2}\right) \int_{0}^{\infty} e^{-\mu t} d t=e^{-\mu \tau} 2 \frac{C_{1}}{\mu} \bar{r} d\left(\rho_{1}, \rho_{2}\right),
\end{aligned}
$$

and

$$
d\left(\tilde{\rho}_{1}, \tilde{\rho}_{2}\right) \leq 2 \frac{C_{1}}{\mu} \bar{r} d\left(\rho_{1}, \rho_{2}\right)
$$

and, using also (4.16), we conclude that $\mathcal{K}_{\bar{r}}$ is a contraction on $R_{\bar{r}}$ if

$$
\bar{r} \leq \mu \min \left\{\frac{1}{C}, \frac{1}{2 C_{1}}\right\} .
$$


From (4.15) with $\rho=\rho^{*}$ we have

$$
\frac{\rho^{*}(\tau)}{e^{-\mu \tau}}=\bar{r}+\int_{0}^{\tau} e^{\mu t} g\left(\rho^{*}(t)\right) d t .
$$

The integral on the right hand side is absolutely convergent. Therefore if we set $\tilde{r}=$ $\int_{0}^{\infty} e^{\mu t} g\left(\rho^{*}(t)\right) d t$ we can write

$$
\frac{\rho^{*}(\tau)}{e^{-\mu \tau}}=\bar{r}+\tilde{r}-\int_{\tau}^{\infty} e^{\mu t} g\left(\rho^{*}(t)\right) d t .
$$

From $\left|\rho^{*}(\tau)\right| \leq 2 \bar{r} e^{-\mu \tau}$ and (4.14) it follows

$$
\left|\int_{\tau}^{\infty} e^{\mu t} g\left(\rho^{*}(t)\right) d t\right| \leq \frac{4 C \bar{r}^{2}}{\mu} e^{-\mu \tau},
$$

and we have

$$
r(s)=\rho^{*}(s-\bar{s})=(\bar{r}+\tilde{r}) e^{-\mu(s-\bar{s})}+\omega(s)=\bar{K}^{+} e^{-\mu s}+\omega(s),
$$

where $\bar{K}^{+}=(\bar{r}+\tilde{r}) e^{\mu \bar{s}}$ and

$$
\begin{aligned}
& \omega(s)=e^{-\mu(s-\bar{s})} \int_{(s-\bar{s})}^{\infty} e^{\mu t} g\left(\rho^{*}(t)\right) d t, \\
& |\omega(s)| \leq \frac{4 C \bar{r}^{2}}{\mu} e^{2 \mu \bar{s}} e^{-2 \mu s}, \quad s \geq \bar{s} .
\end{aligned}
$$

If we insert (4.19) into (4.10) we get

$$
\begin{aligned}
& \bar{u}(s)-a=\bar{K}^{+} e^{-\mu s} z+w(s), \quad s \geq \bar{s}, \\
& w(s)=\omega(s) z+v(r(s)) .
\end{aligned}
$$

From the previous estimate for $\omega$ and $v(r)=\mathrm{O}\left(r^{2}\right)$ it follows

$$
|w(s)| \leq K^{+} e^{-2 \mu s}, \quad s \geq \bar{s},
$$

for some $K^{+}>0$. Actually, by trivially extending the definition of $w$ to the whole $[0,+\infty)$ by setting

$$
w(s)=\bar{u}(s)-a-\bar{K}^{+} e^{-\mu s} z, \quad s \in[0, \bar{s})
$$

we can assume that the expression of $\bar{u}(s)-a$ in (4.20) is valid for all $s \geq 0$. Since $w$ is a bounded map, by increasing the constant $K^{+}$if necessary, we can also assume that the estimate (4.21) is valid for all $s>0$. Similar arguments based on the fact that $D^{k} \rho$ solves the $k$-derivative of (4.15) complete the proof.

\section{References}

[1] F. Alessio and P. Montecchiari, Brake orbits type solutions to some class of semilinear elliptic equations, Calc. Var. Part. Diff. Eqs. 30, 1 (2007), pp. 51-83.

[2] F. Alessio, P. Montecchiari and A. Zuniga, Prescribed Energy Connecting Orbits for Gradient Systems, Discr. Cont. Dyn. Syst. 38 n.8, (2019), pp. 4895-4928. 
[3] N. D. Alikakos, P. Bates and G. Fusco, Slow motion for the Cahn-Hilliard equation in one space dimension, J. Diff. Eqs. 90 (1991), pp. 81-131.

[4] N. D. Alikakos, S. I. Betelu, and X. Chen, Explicit stationary solutions in multiple well dynamics and non-uniqueness of interfacial energy densities, European J. Appl. Math. 17 (2006), pp. 525-556.

[5] N. D. Alikakos, L. Bronsard and G. Fusco, Slow motion in the gradient theory of phase transitions via energy and spectrum, Calc. Var. Part. Diff. Eqs. 6, 1 (1998), pp. 39-66.

[6] N. D. Alikakos and G. Fusco, On the connection problem for the potentials with several global minima. Indiana Univ. Math. Journal 57, 4 (2008), pp. 1871-1906.

[7] N. Alikakos and G. Fusco: Slow dynamics for the Cahn-Hilliard equation in higher space dimensions: The motion of bubbles. Arch. Rational Mech. Anal. 141 (1998), pp. 1-61.

[8] N. D. Alikakos, G. Fusco and M. Kowalczyk, Finite Dimensional Dynamics and Interfaces Intersecting the Boundary: Equilibria and Quasi-Invariant Manifold, Indiana Univ. Math. J. 45, 4 (1996), pp. 1119-1155.

[9] A. Antonopoulos and P. Smyrnelis On minimizers of the Hamiltonian system $u^{\prime \prime}=$ $\nabla W(u)$ and on the existence of heteroclinic, homoclinic and periodic orbits, Indiana Univ. Math. J. 65, 4 (2016), pp. 1503-1524.

[10] P. W. Bates and J. P. Xun, Metastable patterns for the Cahn-Hilliard equation: Part I, J. Differential Eqs. 111, 2 (1994), pp. 421-457.

[11] P. W. Bates, G. Fusco and G. Karaly, Gradient Dynamics: Motion Near a Manifold of Quasi-Equilibria, SIAM J. Appl. Dyn. Syst., 17 n.3 (2018), pp. 2106-2145.

[12] P. W. Bates, J. Xun, Metastable Patterns for the Cahn-Hilliard Equation: Part II. Layer Dynamics and Slow Invariant Manifold, J. Diff. Eqs. 117, (1995), pp. 165-216.

[13] G. Bellettini, A. Nayam, A. and M. Novaga, $\Gamma$-type estimates for the one-dimensional Allen-Cahn's action. Asymptot. Anal. 94 no. 1-2, (2015), pp. 161-185

[14] F. Bethuel, G. Orlandi and D. Smets, Slow motion for gradient systems with equal depth multiple-well potentials, J. Diff. Eqs. 250 no. 1, (2011), pp. 53 - 94.

[15] F. Bethuel, D. Smets, Slow motion for equal depth multiple-well gradient systems: The degenerate case, $D C D S-A$, 33, no 1, (2013), pp. 67 - 87.

[16] L. Bronsard, R. V Kohn., On the slowness of phase boundary motion in one space dimension, Comm. Pure. Appl. Math. 43 (1990), pp. 983-997.

[17] L. Bronsard, D. Hilhorst, On the slow dynamics for the Cahn-Hilliard equation in one space dimension, Proc. Roy. Soc. London A 439 (1992), pp. 669-682.

[18] J. Carr and R. L. Pego, Metastable Patterns in Solutions of $u_{t}=\epsilon^{2} u_{x x}-f(u)$, Comm. Pure Appl. Math. 42 (1989), pp. 523-576.

[19] J. Carr, R. L. Pego, Invariant manifolds for metastable patterns in $u_{t}=\epsilon^{2} u_{x x}-f(u)$, Proc. Roy. Soc. Edin. 116(A) (1990), pp. 133-160. 
[20] S. I. Ei and E. Yanagida, Slow dynamics of interfaces in the Allen-Cahn equation on a strip-like domain. SIAM J. Math. Anal. 29(3), (1998), pp. 555-595. (electronic)

[21] R. Folino, C. Lattanzio and C. Mascia, Slow dynamics for the hyperbolic CahnHilliard equation in one space dimension. Math. Meth. Appl. Sc. 42 (8), (2019), pp. $2492-2512$.

[22] G. Fusco, A Geometric Approach to the Dynamics of $u_{t}=\epsilon^{2} u_{x x}+f(u)$ for small $\epsilon$, Problems Involving Change of Type K. Kirchgässner Ed. Lecture Notes Physics 359 (1990), pp. 53-73.

[23] G. Fusco, G.F. Gronchi and M. Novaga: Existence of periodic orbits near heteroclinic connections. Minimax Theory Appl. 4 n.1 (2019), pp. 113-149.

[24] G. Fusco and J. K. Hale, Slow motion manifolds, dormant instability and singular perturbations, J. Dyn. Diff. Eqs. 1 (1989), pp. 75-94.

[25] C. Grant, Slow motion in one-dimensional Cahn-Morral systems, SIAM. J. Math. Anal. 26 (1995), pp. 21-34.

[26] C. Grant, Spinodal decomposition for the Cahn-Hilliard equation, Comm. Part. Diff. Eqs. 18 (1993), pp. 453-490.

[27] C. Mascia and M. Strani, Metastability for nonlinear parabolic equations with application to scalar viscous conservation laws, SIAM J. Math. Anal. 45, no.9 (2013), pp. 3084-3113.

[28] H. Matano and P. Polácik. Dynamics of nonnegative solutions of one-dimensional reaction-diffusion equations with localized initial data. Part I: A general quasiconvergence theorem and its consequences. Comm. Part. Diff. Eqs. 41 (5) (2016), pp. 1-27.

[29] H. Matano and P. Polácik. Dynamics of nonnegative solutions of one-dimensional reaction-diffusion equations with localized initial data. Part II: Generic nonlinearities. Comm. Part. Diff. Eqs. 45 (6) (2019), pp. 483-524.

[30] A. Monteil and F. Santambrogio. Metric methods for heteroclinic connections. Math. Meth. Appl. Sci. 41 (3) (2016), pp. 1019-1024.

[31] R. Murray and M. Rinaldi. Slow motion for the nonlocal Allen-Cahn equation in n-dimensions. Calc. Var. 55, 147 (2016). https://doi.org/10.1007/s00526-016-1086-4

[32] J. Neu, Unpublished lecture notes.

[33] F. Otto and M. G. Reznikoff, Slow motion of gradient flows, J. Diff. Eqs. 237, (2007), pp. $372-420$.

[34] P. Smyrnelis, Connecting orbits in Hilbert spaces and application to PDE. Comm. Pure Appl. Analysis 19 n.5, (2019), pp. 2797-2818. doi 10.3934/cpaa.2020122

[35] P. Rabinowitz. Homoclinic and heteroclinic orbits for a class of Hamiltonian systems. Calc. of Var. P.D.E. Calc. Var. P.D.E. No. 1, (1993), pp. 1-36.

[36] C. Sourdis, The heteroclinic connection problem for general double-well potentials, Mediterr. J. Math. 13 (2016), pp. 4693-4710. https://doi.org/10.1007/s00009-0160770-0 
[37] M. J. Ward, Metastable patterns, layers collapses and coarsening for a one dimensional Ginzburg-Landau equation, Stud. Appl. Math. 91 (1994), pp. 51-93.

[38] M. J. Ward, Metastable dynamics and exponential asymptotics in multi-dimensional domains, Multiple-Time-Scale Dynamical Systems C.K.R.T. Jones and A.I. Khibnik Edrs. IMA Volum. Math. Applic. 122 (2001), pp. 233-259.

[39] A. Zuniga and P. Sternberg. On the heteroclinic connection problem for multi-well gradient systems. J. Diff. Eqs. 261 n.7 (2016), pp. 3987-4007. https://doi.org/10.1016/j.jde.2016.06.010 